\title{
Sulfonyl Azides as Precursors in Ligand-Free Palladium-Catalyzed Synthesis of Sulfonyl Carbamates and Sulfonyl Ureas and Synthesis of Sulfonamides
}

\author{
Shiao Y. Chow ${ }^{a}$, Marc Y. Stevens ${ }^{a}$, Luke R. Odell $^{*}$
}

Organic Pharmaceutical Chemistry, Department of Medicinal Chemistry, Uppsala Biomedical Center, Uppsala University, P. O. Box 574, SE-751 23 Uppsala, Sweden

\section{Contents}

Reaction setup for preparation of sulfonyl ureas and sulfonyl carbamates from sulfonyl azides .....S3

Stability assay of $p$-nitrobenzenesulfonyl chloride and $p$-nitrobenzenesulfonyl azide at ambient and elevated temperature .S4

$N$-butyl-4-methylbenzenesulfonamide (Table 1, entry 8) (2a) ..................................................S5

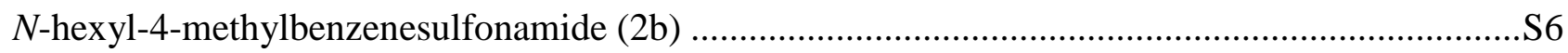

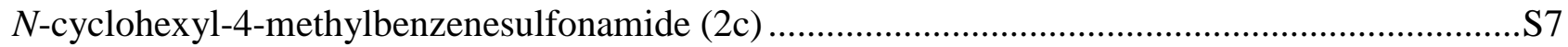

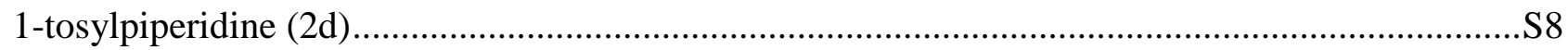

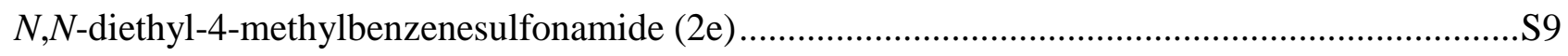

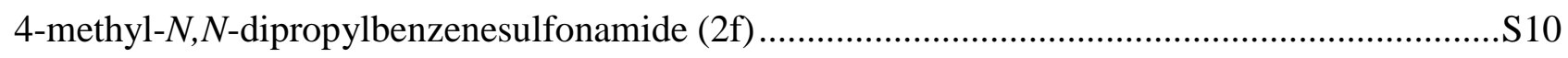

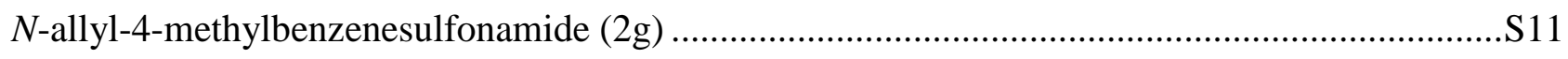

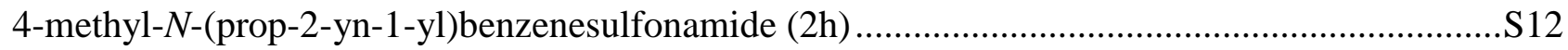

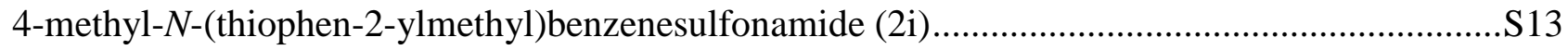

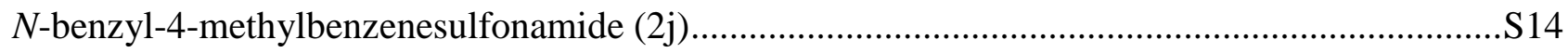

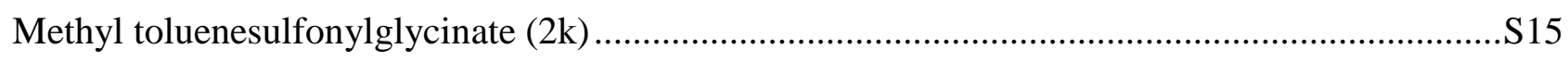

Methyl 3-((4-methylphenyl)sulfonamido)propanoate (2n) ..................................................S16

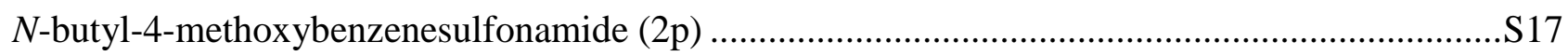

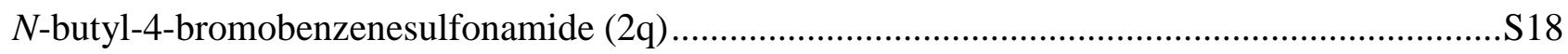

4-Acetyl- $N$-butylbenzenesulfonamide (2r), Table 2 prepared using standard conditions ...............S19

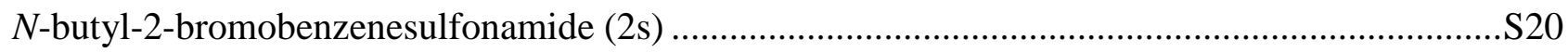

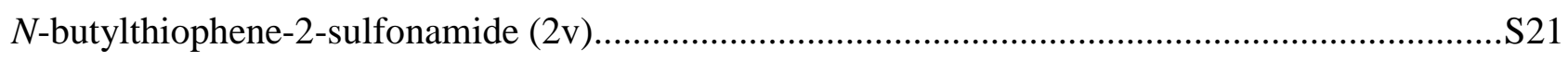

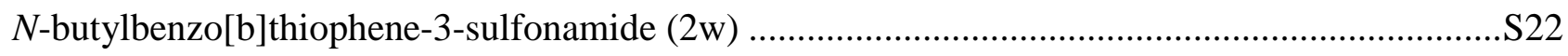

5-(N-butylsulfamoyl)-4-chloro-2-((furan-2-ylmethyl)amino)benzoic acid (2x) ..........................S23

4-Methyl- $N$-(phenylcarbamoyl)benzenesulfonamide (Table 3, entry 3) (3a)..............................S24 
Triethylammonium $N$-((4-methoxyphenyl)carbamoyl)-4-methylbenzenesulfonamide (3b) S25

Triethylammonium $N$-((4-bromophenyl)carbamoyl)-4-methylbenzenesulfonamide (3c)..............S26

Triethylammonium $N$-((4-chlorophenyl)carbamoyl)-4-methylbenzenesulfonamide (3d)..............S27

Triethylammonium $N$-((4-cyanophenyl)carbamoyl)-4-methylbenzenesulfonamide (3e) ..............S28

Triethylammonium methyl 4-(3-tosylureido)benzoate (3f) ......................................................S29

Triethylammonium 4-methyl- $\mathrm{N}$-(o-tolylcarbamoyl)benzenesulfonamide (3g) ............................S30

Triethylammonium $N$-((2-chlorophenyl)carbamoyl)-4-methylbenzenesulfonamide (3h)..............S31

Triethylammonium 4-Methoxy- $N$-(phenylcarbamoyl)benzenesulfonamide (3i) .........................S32

Triethylammonium 4-Acetyl- $N$-(phenylcarbamoyl)benzenesulfonamide (3j) ............................S33

5-Chloro- $N$-(phenylcarbamoyl)thiophene-2-sulfonamide (3k) ..............................................S34

Triethylammonium $N$-(Phenylcarbamoyl)benzo[b]thiophene-3-sulfonamide (31).......................S35

Butyl toluenesulfonylcarbamate (Table 5, entry 3) (4a) .........................................................S36

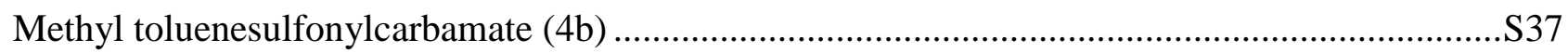

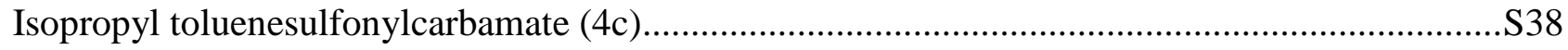

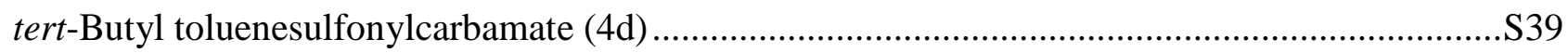

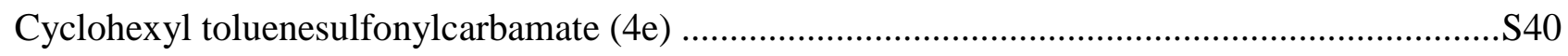

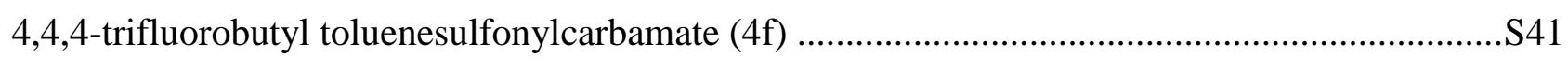

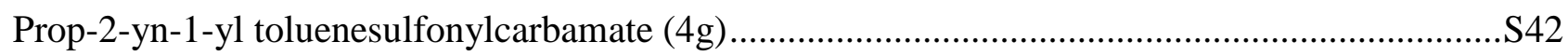

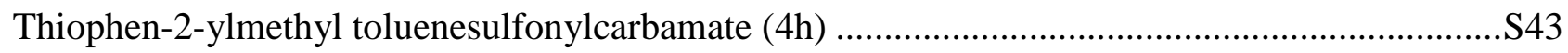

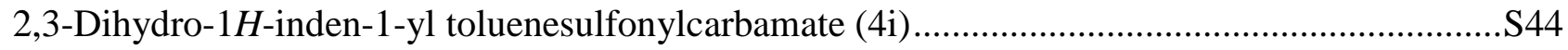

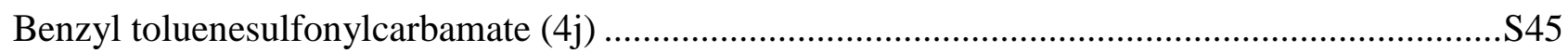

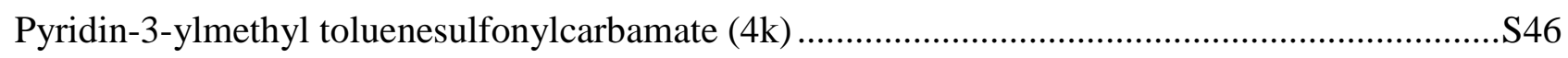

Triethylammonium ((cinnamyloxy)carbonyl)(tosyl)amide (41) ….........................................S47

Triethylammonium (butoxycarbonyl)((4-methoxyphenyl)sulfonyl)amide (4n) .........................S48

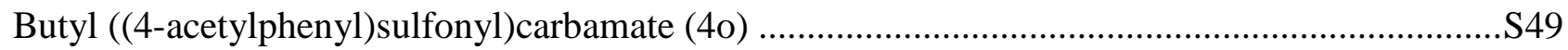

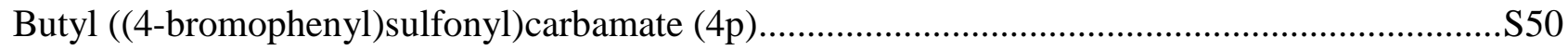

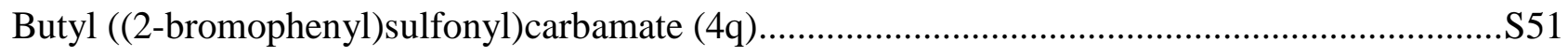

Triethylammonium (butoxycarbonyl)(thiophen-2-ylsulfonyl)amide (4r) .................................S52

Triethylammonium (butoxycarbonyl)((5-chlorothiophen-2-yl)sulfonyl)amide (4s) ....................S53

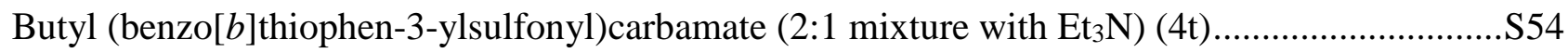

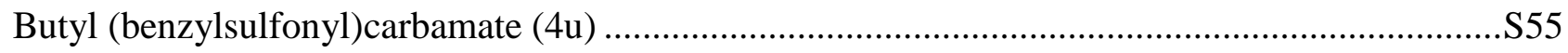

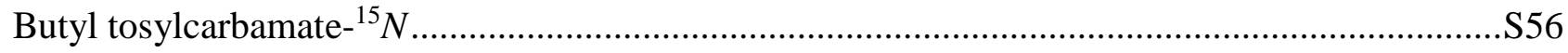

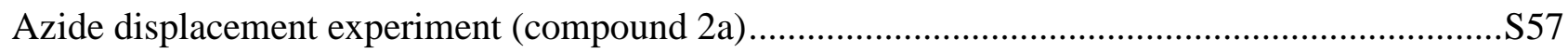




\section{Reaction setup for preparation of sulfonyl ureas and sulfonyl carbamates from sulfonyl azides}

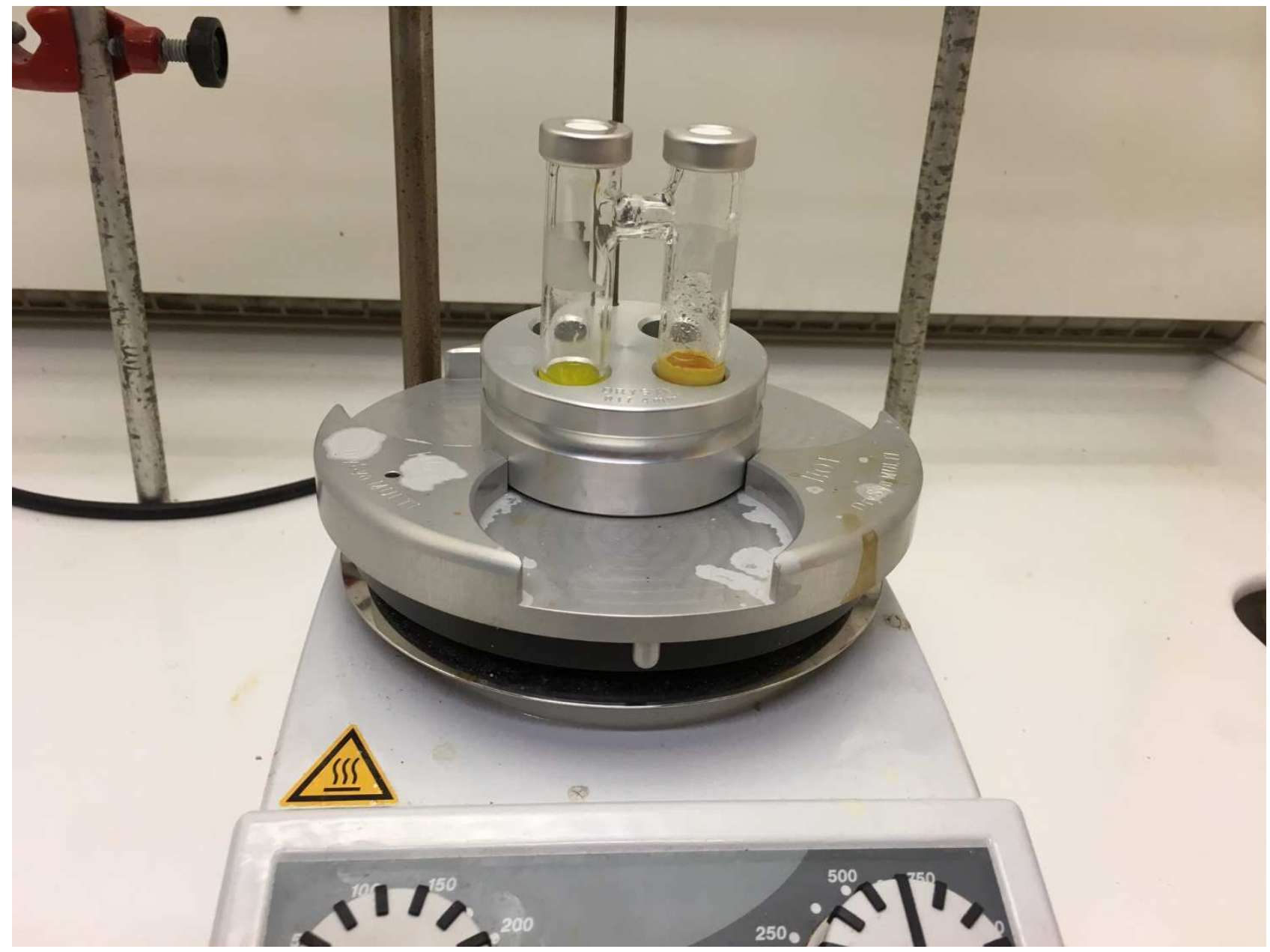

Figure S1. Standard reaction setup for carbonylative synthesis of sulfonyl ureas and sulfonyl carbamates. Left-hand chamber contains sulfonyl azide, nucleophile and $\mathrm{PdCl}_{2}$, right-hand chamber contains $\mathrm{Mo}(\mathrm{CO})_{6}$ and $\mathrm{DBU}$. All reactions were run in DMA. 
Stability assay of $p$-nitrobenzenesulfonyl chloride and $p$-nitrobenzenesulfonyl azide at ambient and elevated temperature

On the suggestion of an anonymous reviewer, a stability assay was conducted to evaluate the stability of electron-deficient arylsulfonyl azides and arylsulfonyl chlorides. Heteroarylsulfonyl chlorides such as 2-pyrimidinesulfonyl chlorides are known to undergo decomposition with loss of $\mathrm{SO}_{2}$ to furnish the corresponding pyrimidine-2-chloride. ${ }^{1}$ Procedure: a standard $5 \mathrm{~mm}$ NMR tube containing $\mathrm{a} \sim 0.05 \mathrm{mM}$ solution of sulfonyl chloride or sulfonyl azide was analyzed ${ }^{2}$ by ${ }^{1} \mathrm{H}$ NMR at $1 \mathrm{~h}$ and $18 \mathrm{~h}$. Procedure $\mathrm{A}=\mathbf{a m b i e n t}$ temperature. Procedure $\mathrm{B}=\mathbf{e l e v a t e d}$ temperature $\left(50{ }^{\circ} \mathrm{C}\right)$. The relative degree of decomposition was calculated by comparing the integrals of compound vs. impurities over time (see Figure S2). The substrates were found to be stable under these conditions and no traces of degraded products were observed.

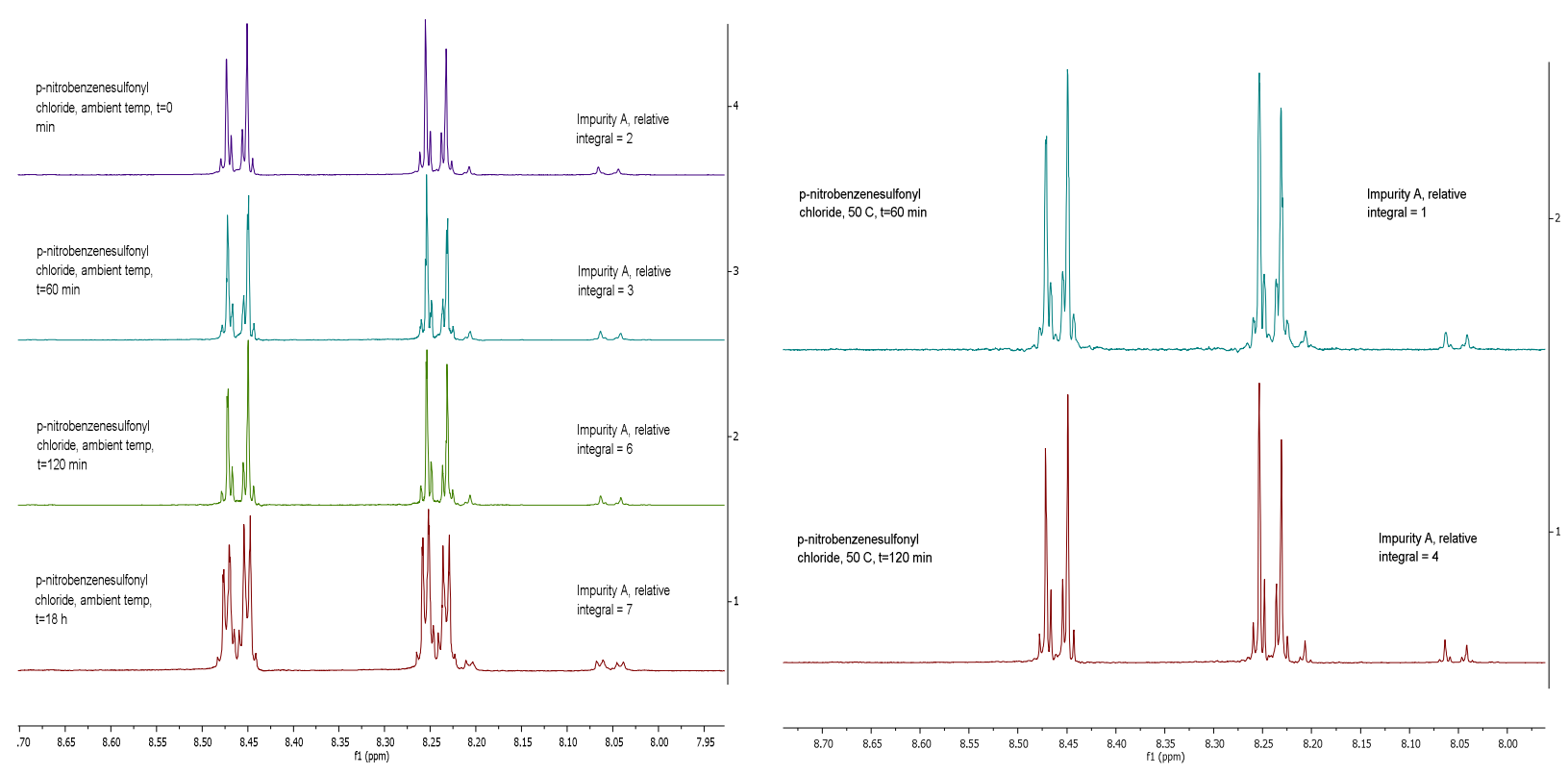

Figure S2. Stability assay of $p$-nitrobenzenesulfonyl chloride in $\mathrm{CDCl}_{3}$ at ambient temperature and $50{ }^{\circ} \mathrm{C}$

\footnotetext{
${ }^{1}$ Brown, D. J. The Chemistry of Heterocyclic Compounds, the Pyrimidines: Supplement 2. Volume 16 of The Chemistry of Heterocyclic Compounds; John Wiley \& Sons: New York, 2009; p.342.

${ }^{2} \mathrm{NMR}$ acquisition parameters: relaxation delay $=60 \mathrm{~s}$, acquisition time $=5.126 \mathrm{~s}$, number of scans $=1$, spectral width $=6398 \mathrm{~Hz}$.
} 
$N$-butyl-4-methylbenzenesulfonamide (Table 1, entry 8) (2a)
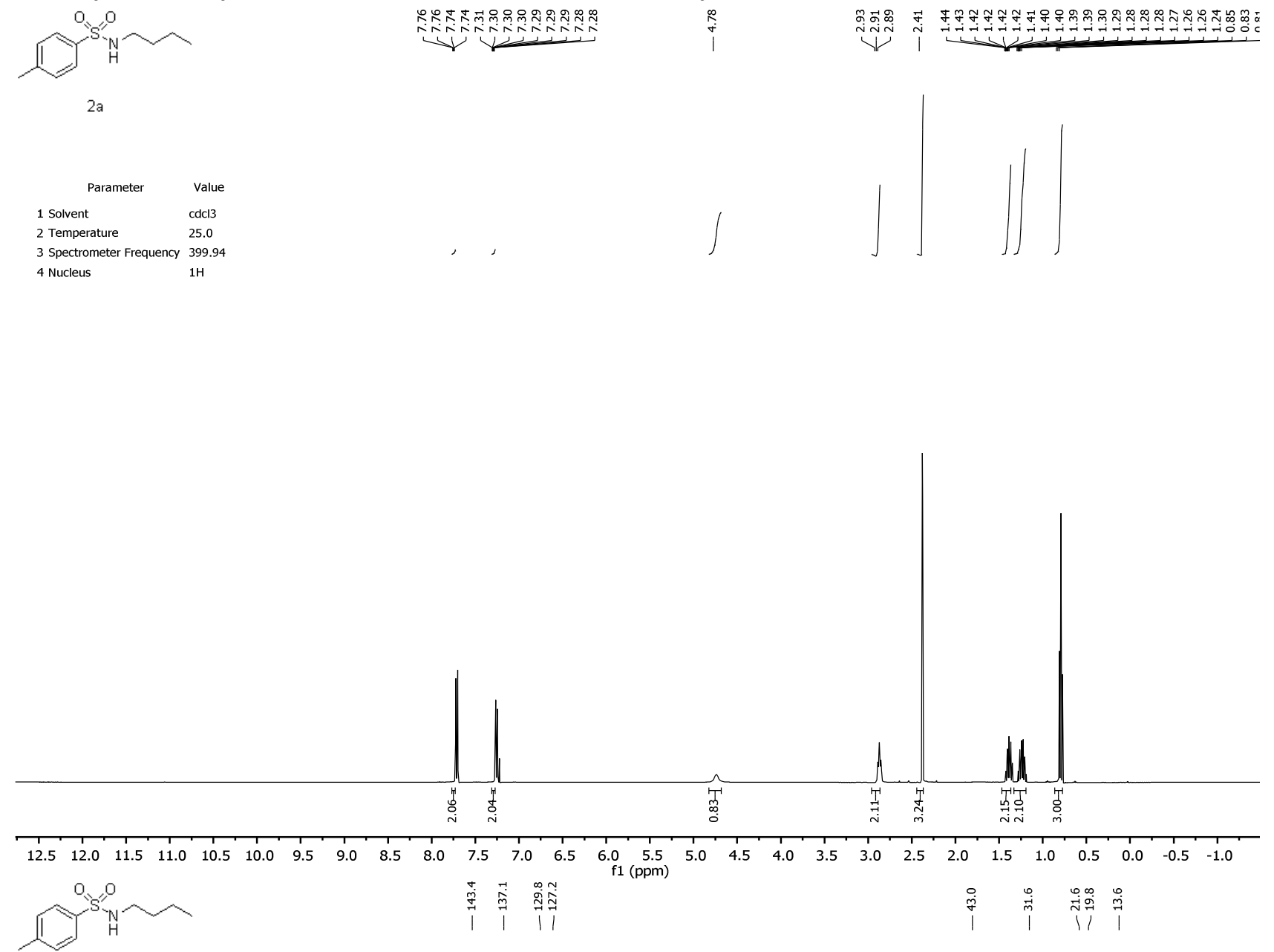

$2 a$

\begin{tabular}{ll}
\multicolumn{1}{c}{ Parameter } & \multicolumn{1}{c}{ Value } \\
1 Solvent & cdcl3 \\
2 Temperature & 25.0 \\
3 Spectrometer Frequency & 100.58 \\
4 Nucleus & $13 \mathrm{C}$
\end{tabular}

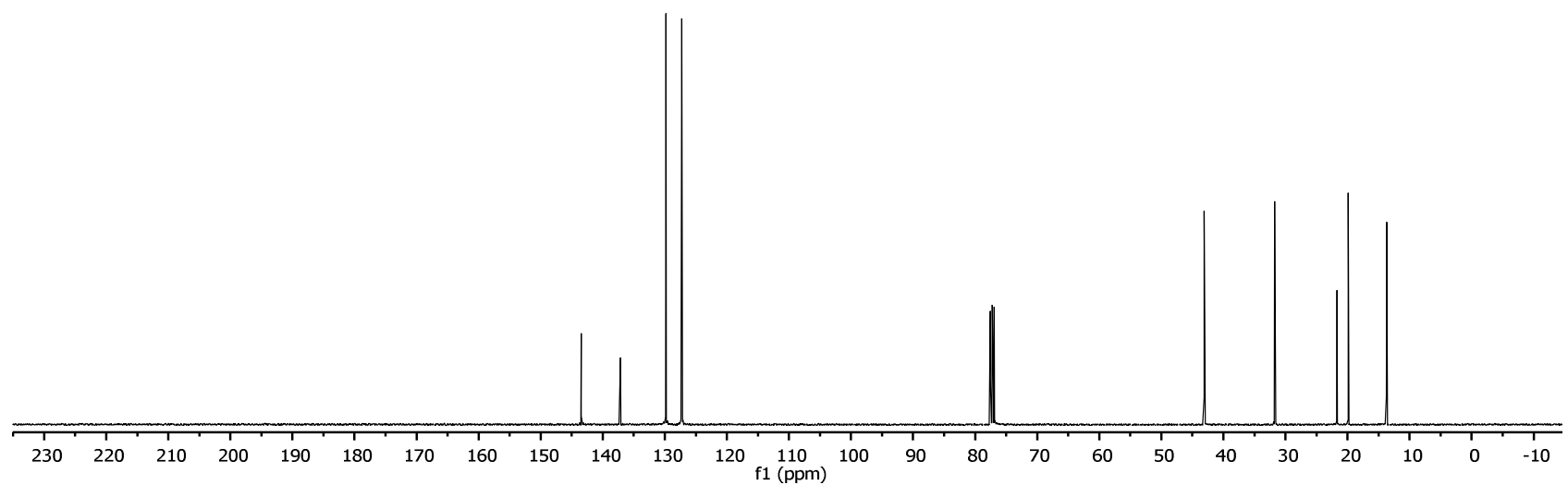


$N$-hexyl-4-methylbenzenesulfonamide (2b)

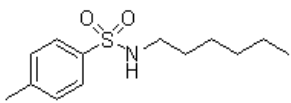

$2 \mathrm{~b}$

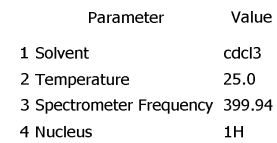

3 Spectrometer Frequency 399

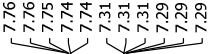

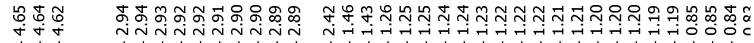

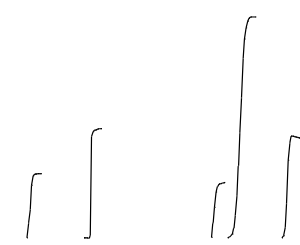

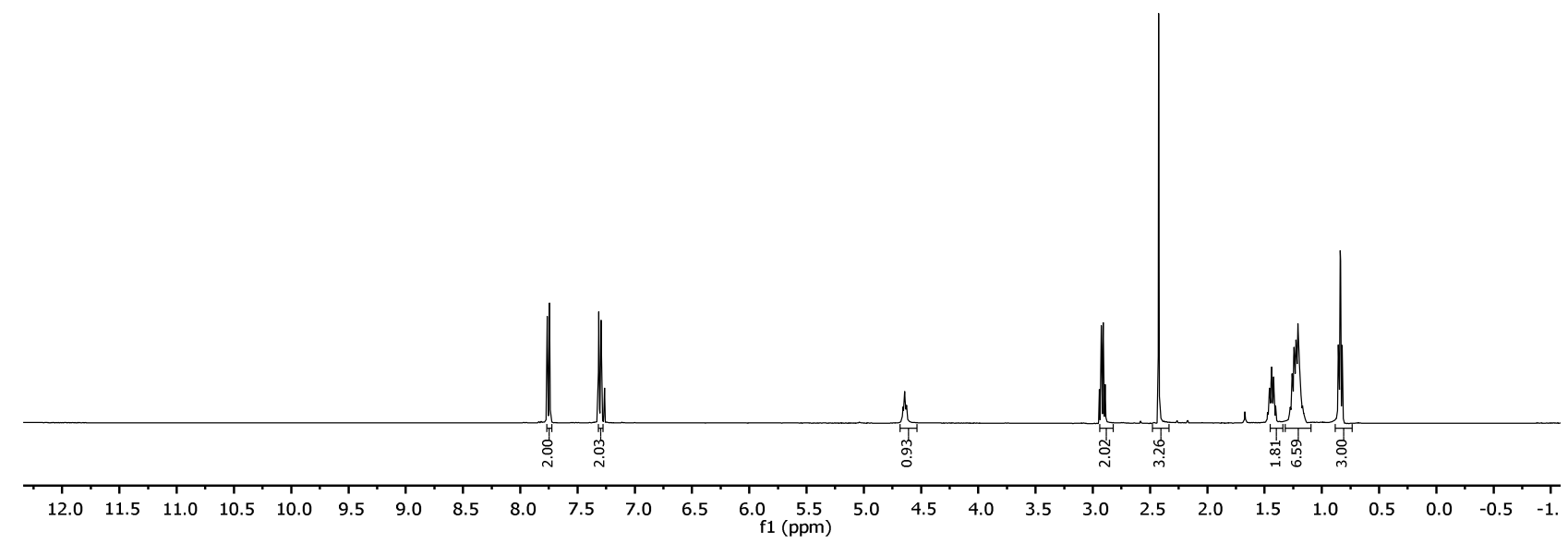

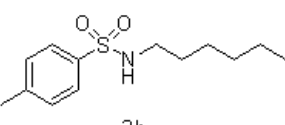

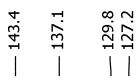

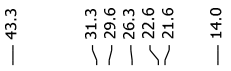
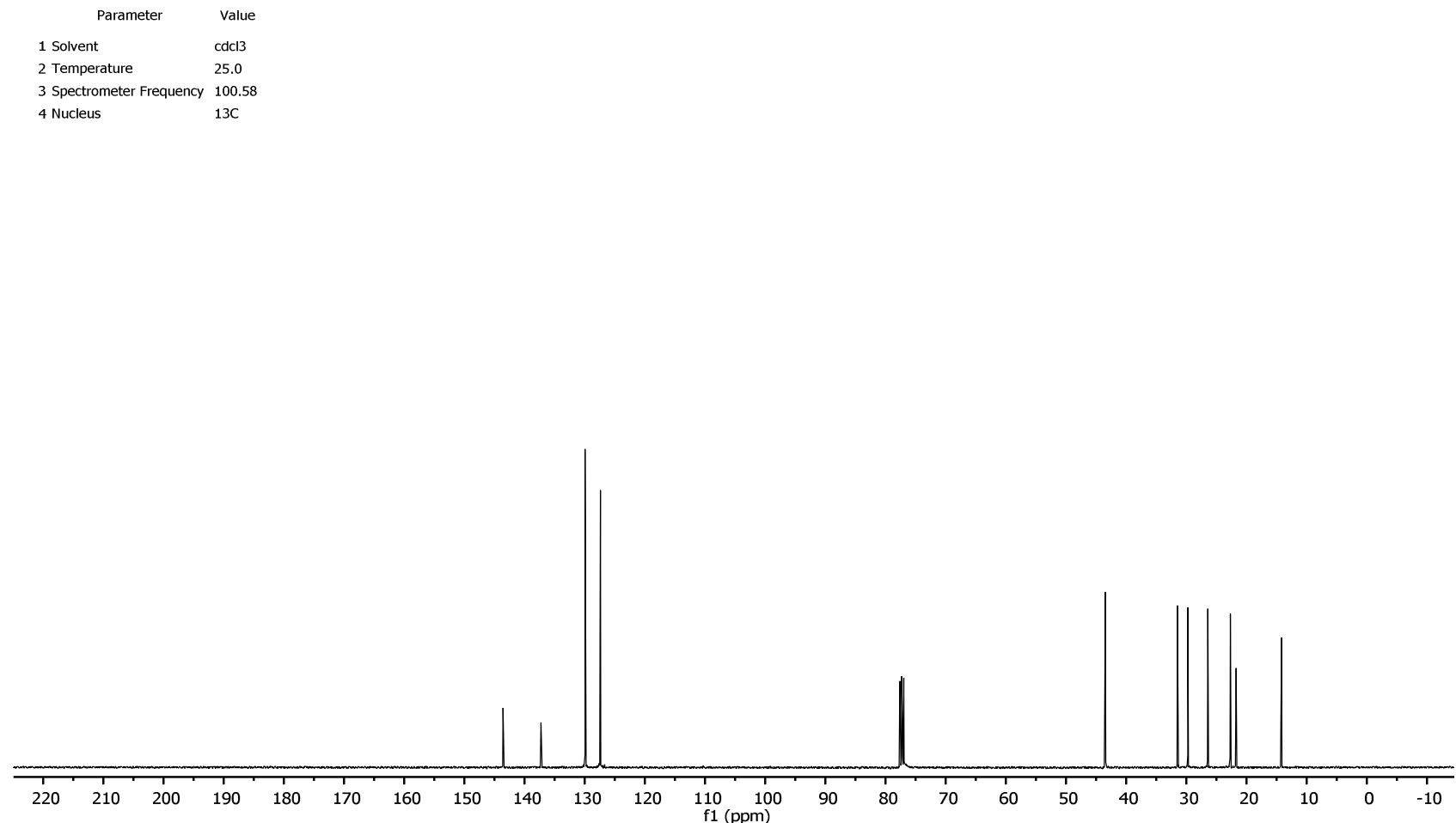
$N$-cyclohexyl-4-methylbenzenesulfonamide (2c)
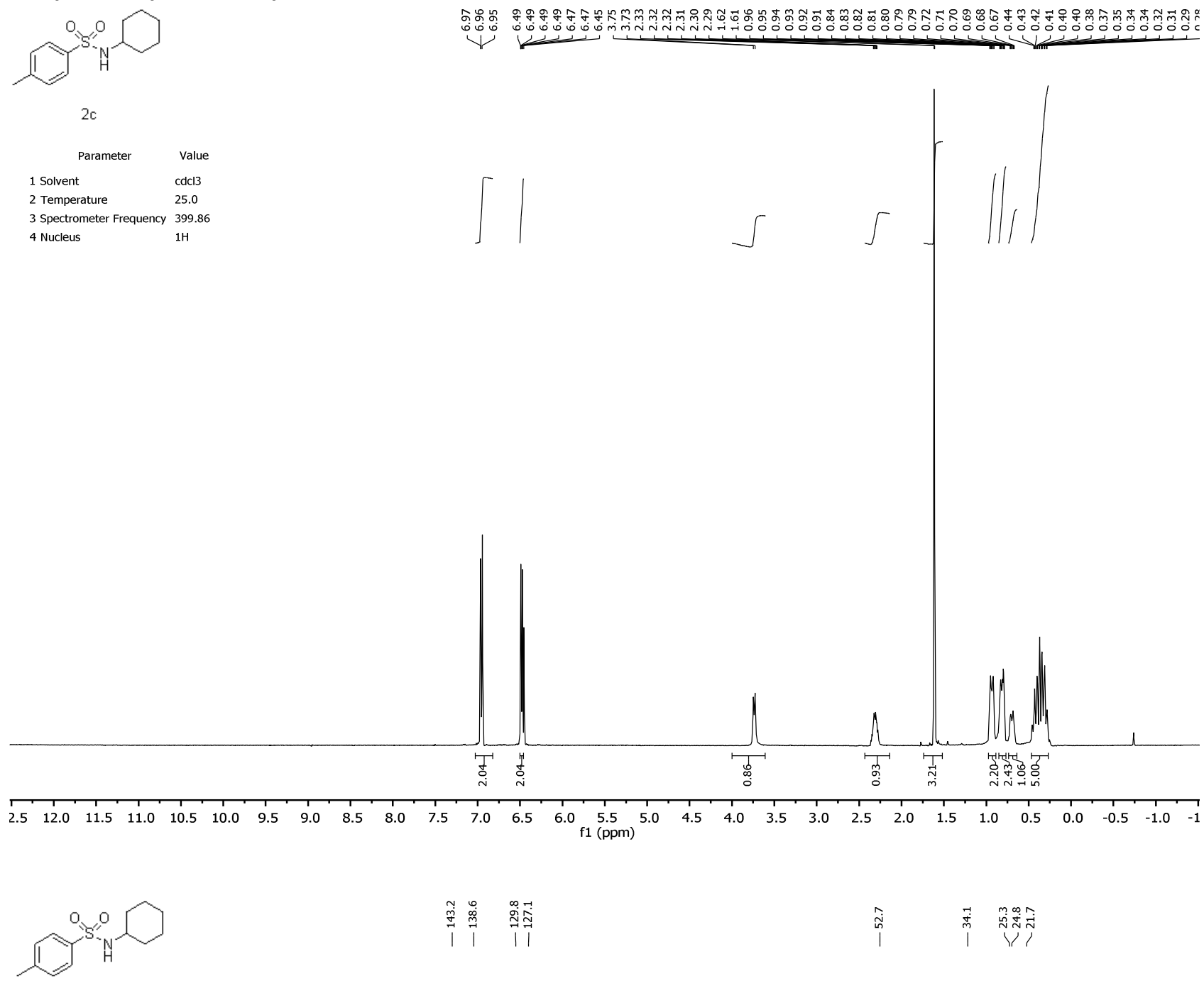

$2 c$

\begin{tabular}{ll}
\multicolumn{1}{c}{ Parameter } & \multicolumn{1}{c}{ Value } \\
1 Solvent & cdl3 \\
2 Temperature & 25.0 \\
3 Spectrometer Frequency & 100.55 \\
4 Nucleus & $13 \mathrm{C}$ \\
& \\
\end{tabular} 
1-tosylpiperidine (2d)

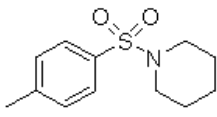

$2 d$
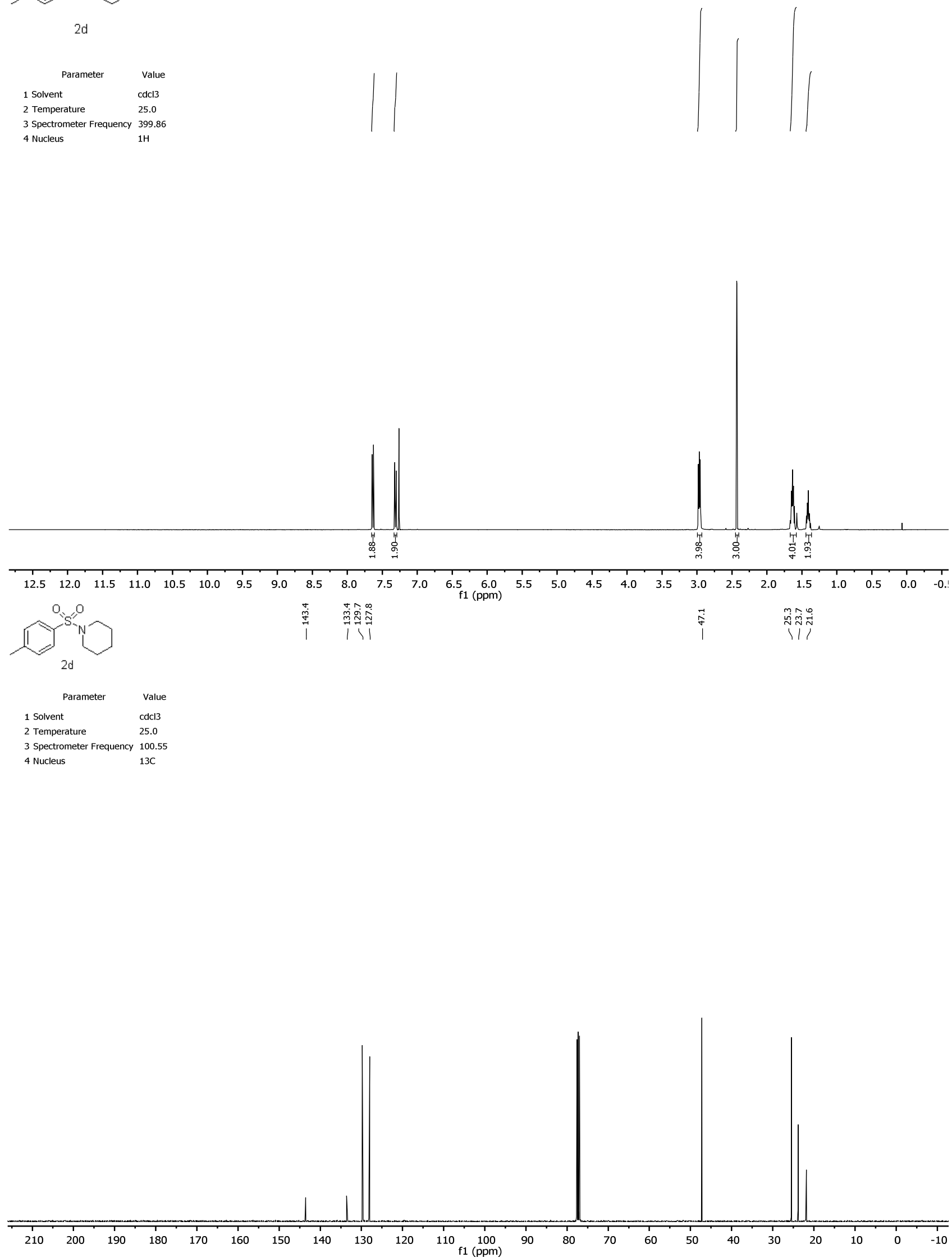
N,N-diethyl-4-methylbenzenesulfonamide (ae)

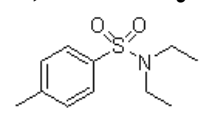

$2 \mathrm{e}$

Parameter

1 Solvent

2 Temperature

3 Spectrometer Frequency 25.0

4 Nucleus

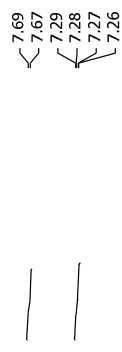

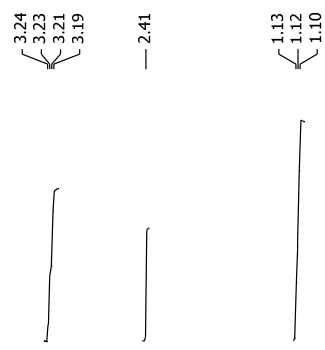
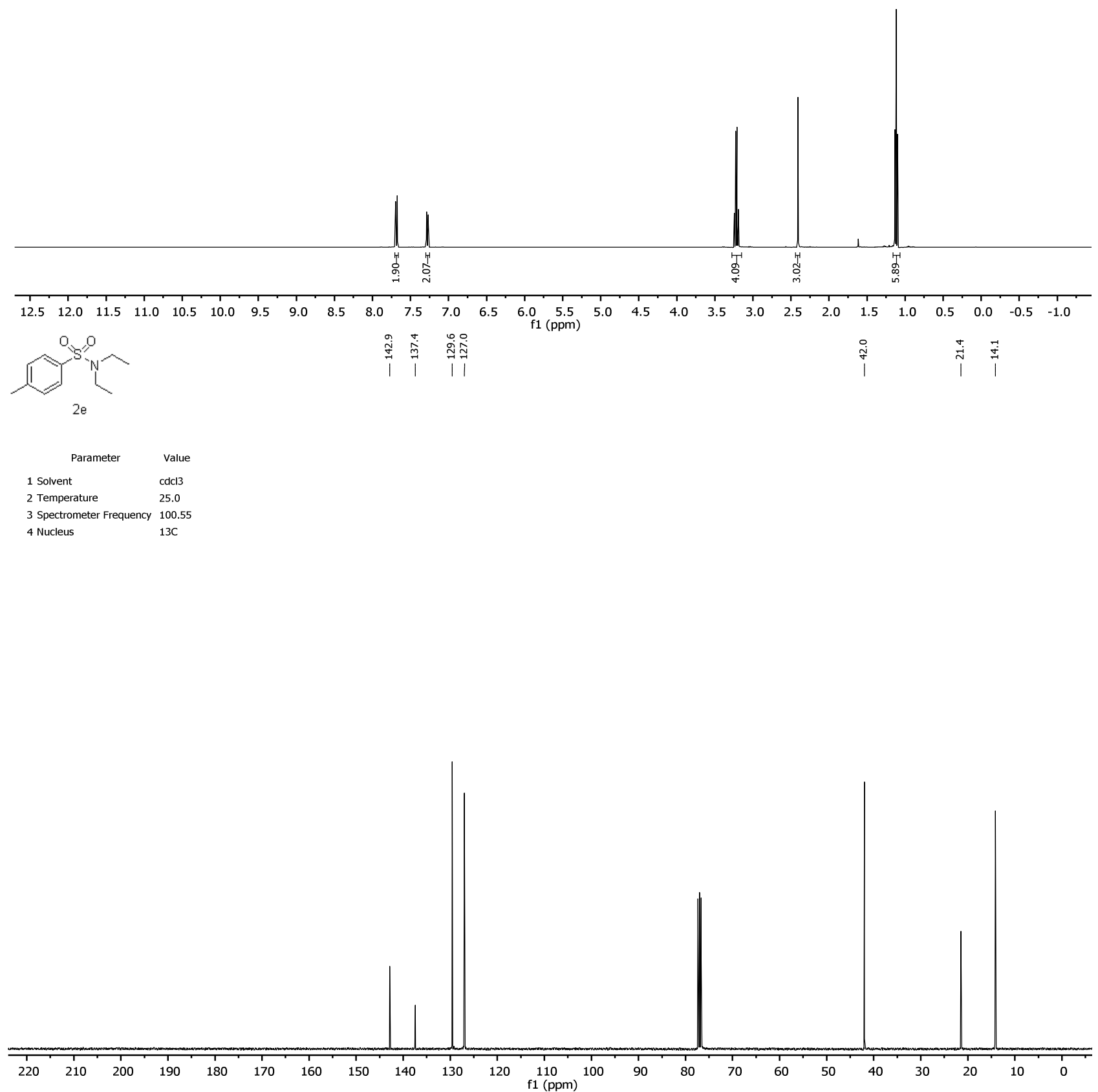

Sg 
4-methyl- $N, N$-dipropylbenzenesulfonamide (2f)
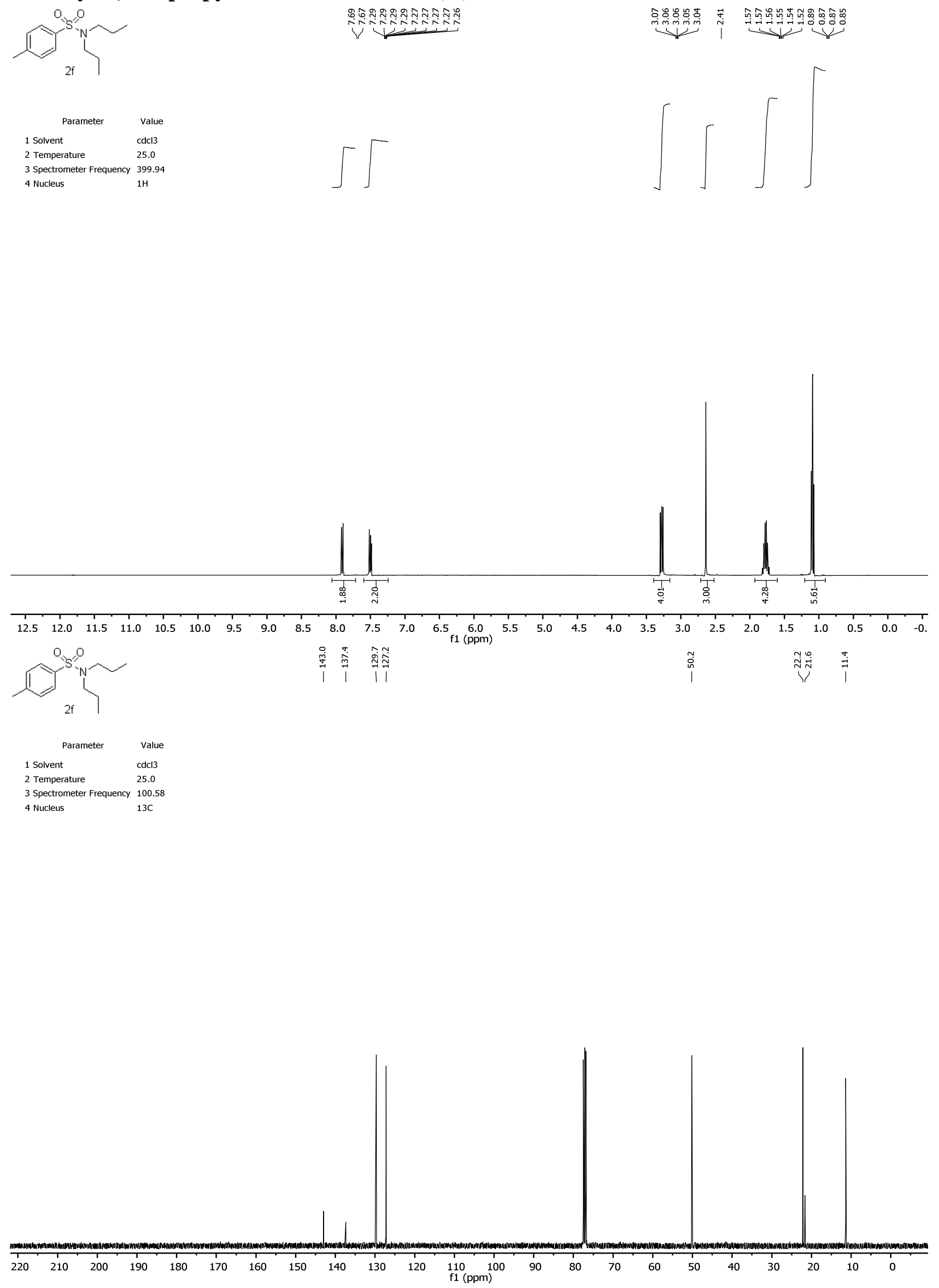


\section{$\mathrm{N}$-allyl-4-methylbenzenesulfonamide (2g)}
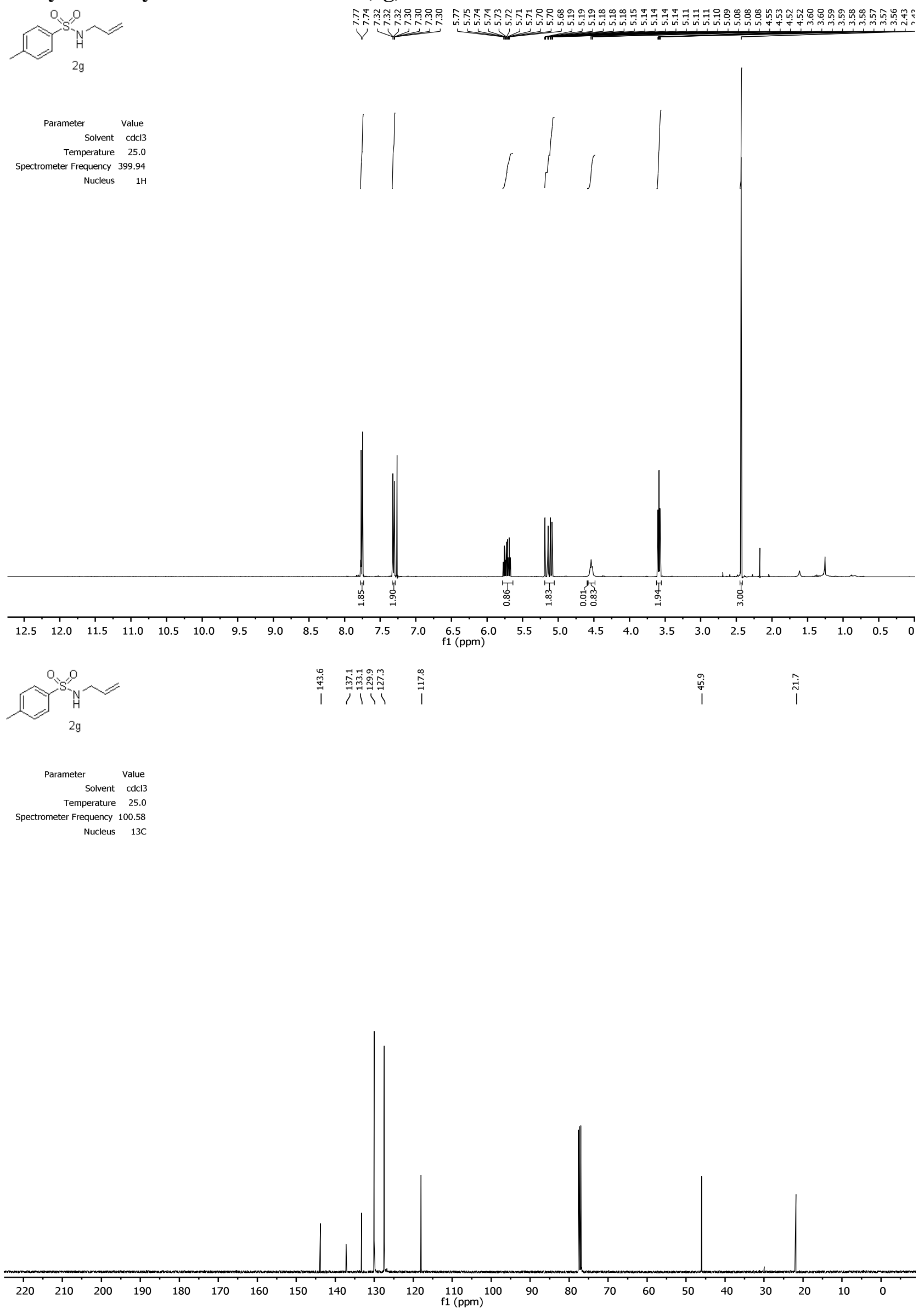
4-methyl- $N$-(prop-2-yn-1-yl)benzenesulfonamide (2h)
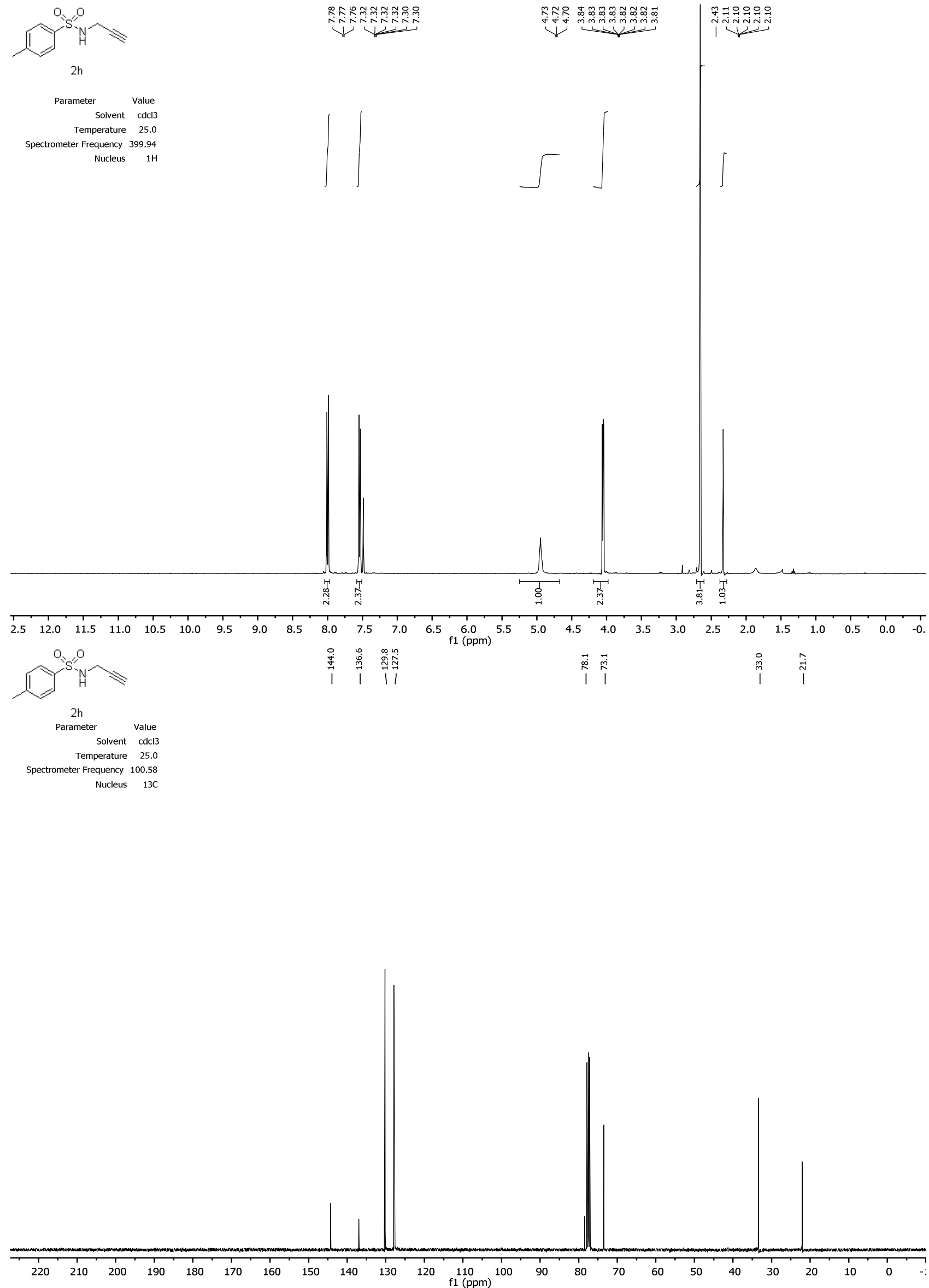
4-methyl- $N$-(thiophen-2-ylmethyl)benzenesulfonamide (2i)
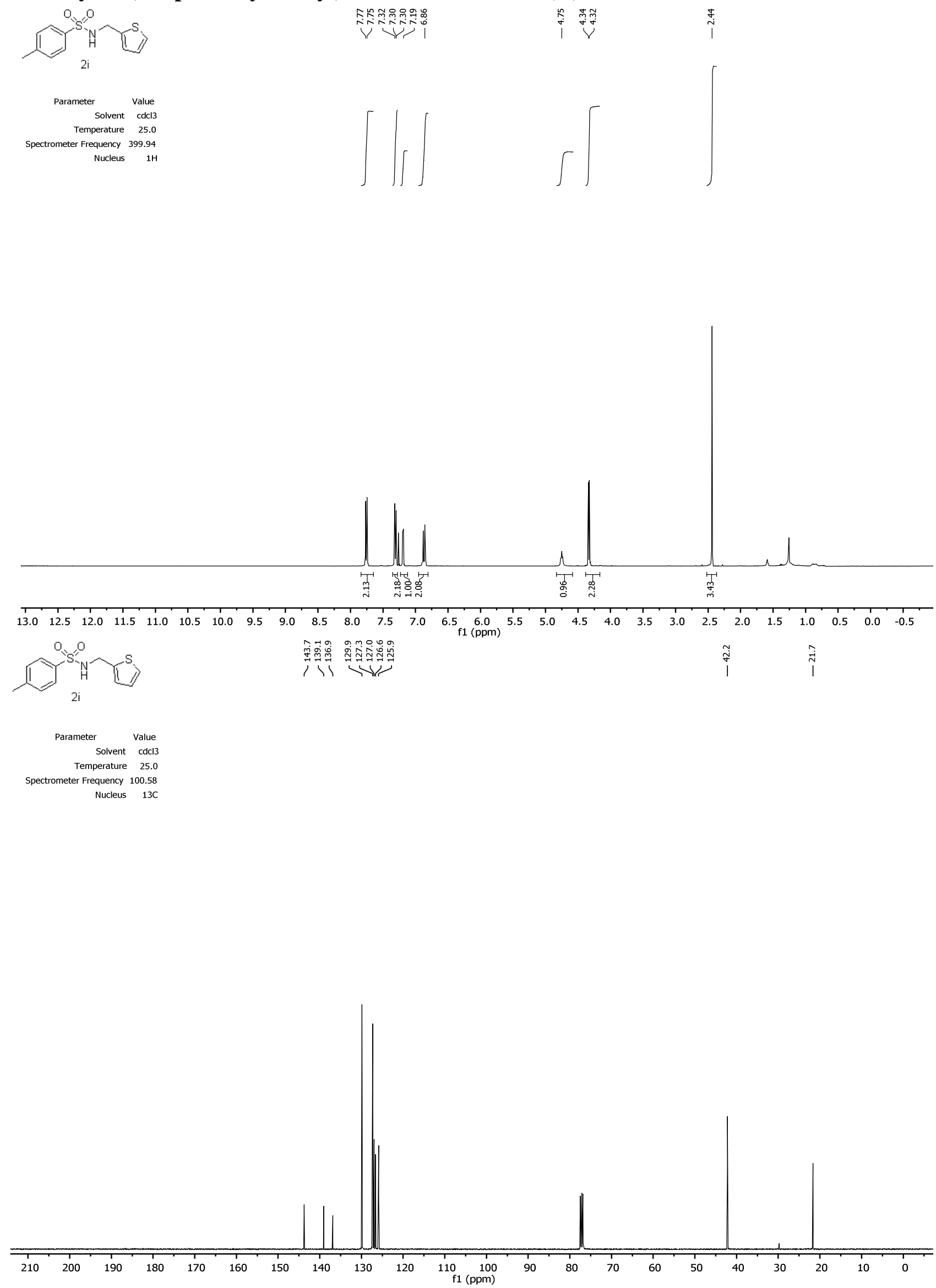
$N$-benzyl-4-methylbenzenesulfonamide (2j)

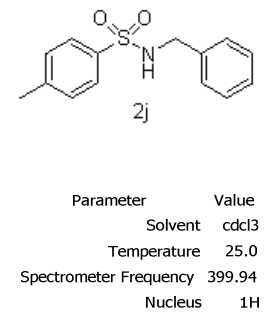

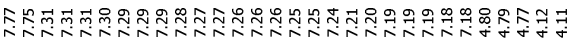
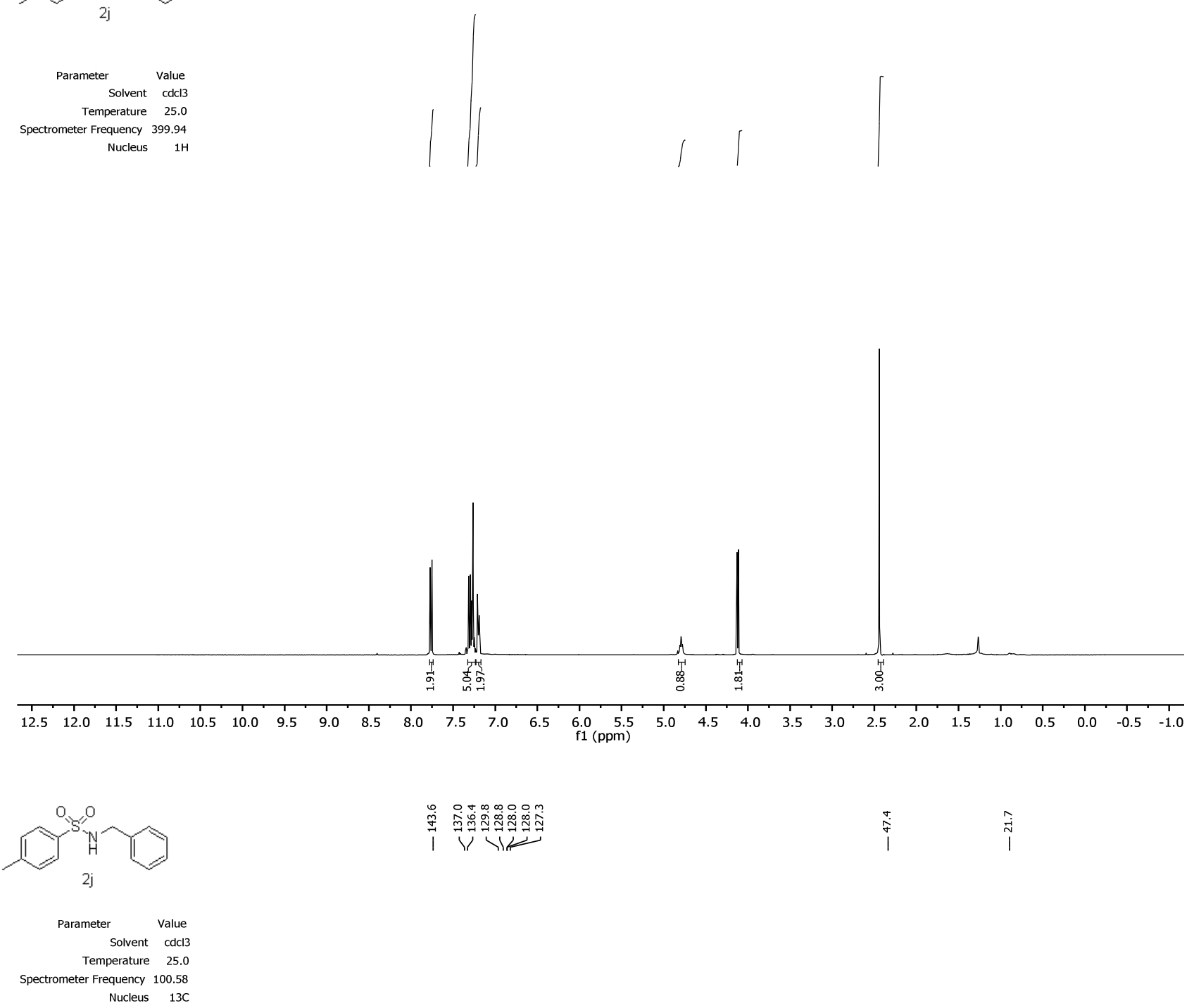

।

(ppm) 
Methyl toluenesulfonylglycinate (2k)
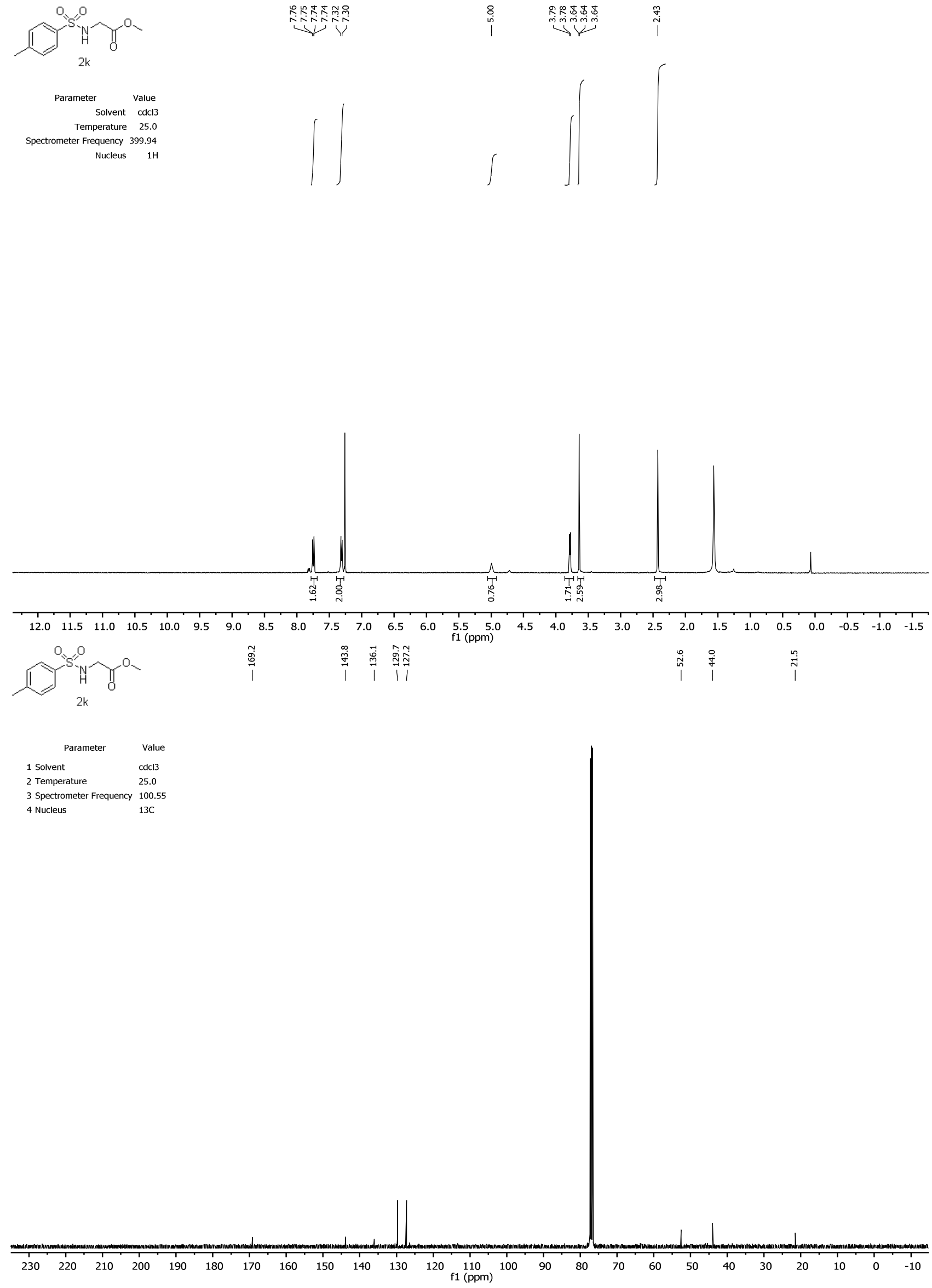
Methyl 3-((4-methylphenyl)sulfonamido)propanoate (2n)
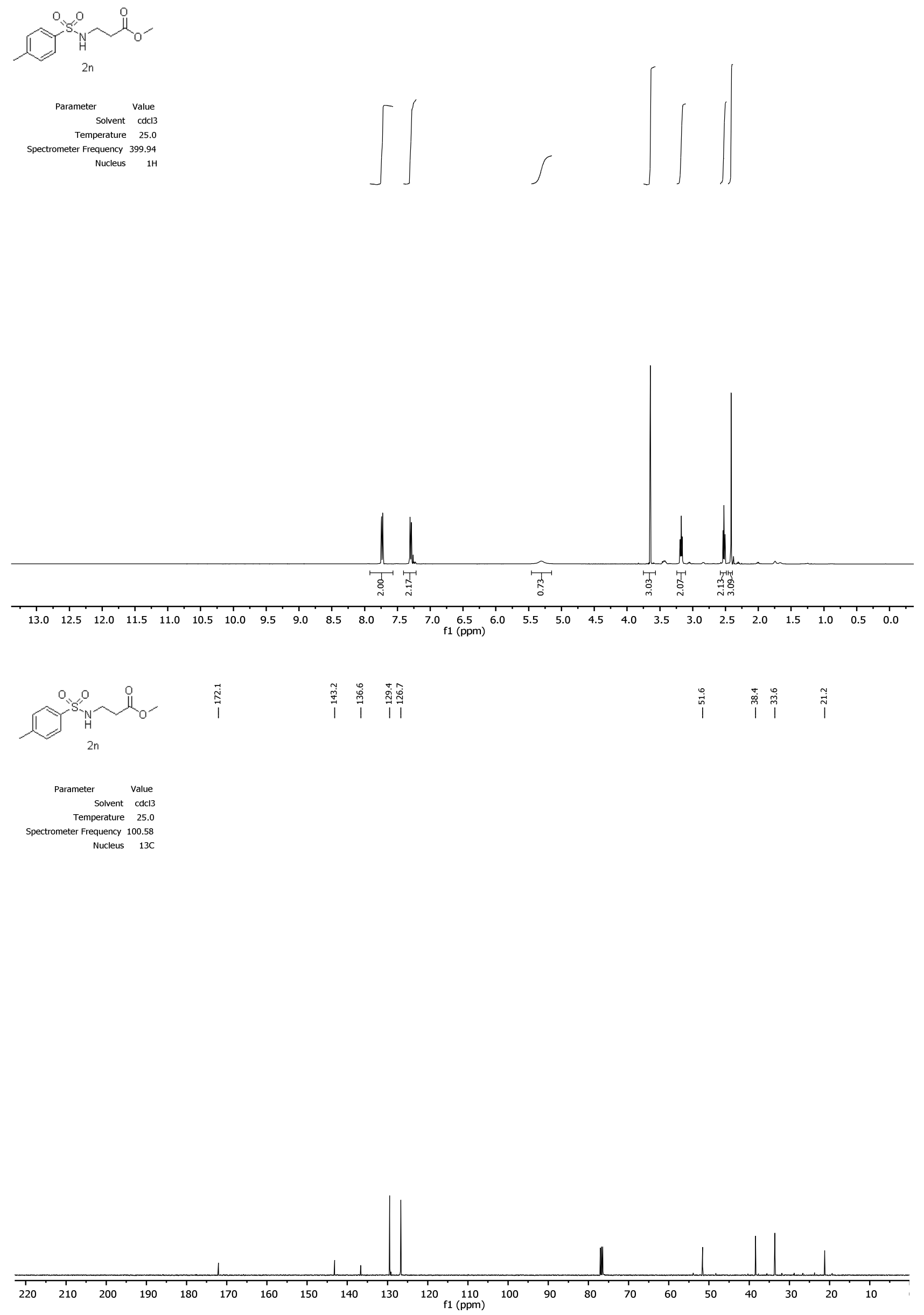
$N$-butyl-4-methoxybenzenesulfonamide (2p)
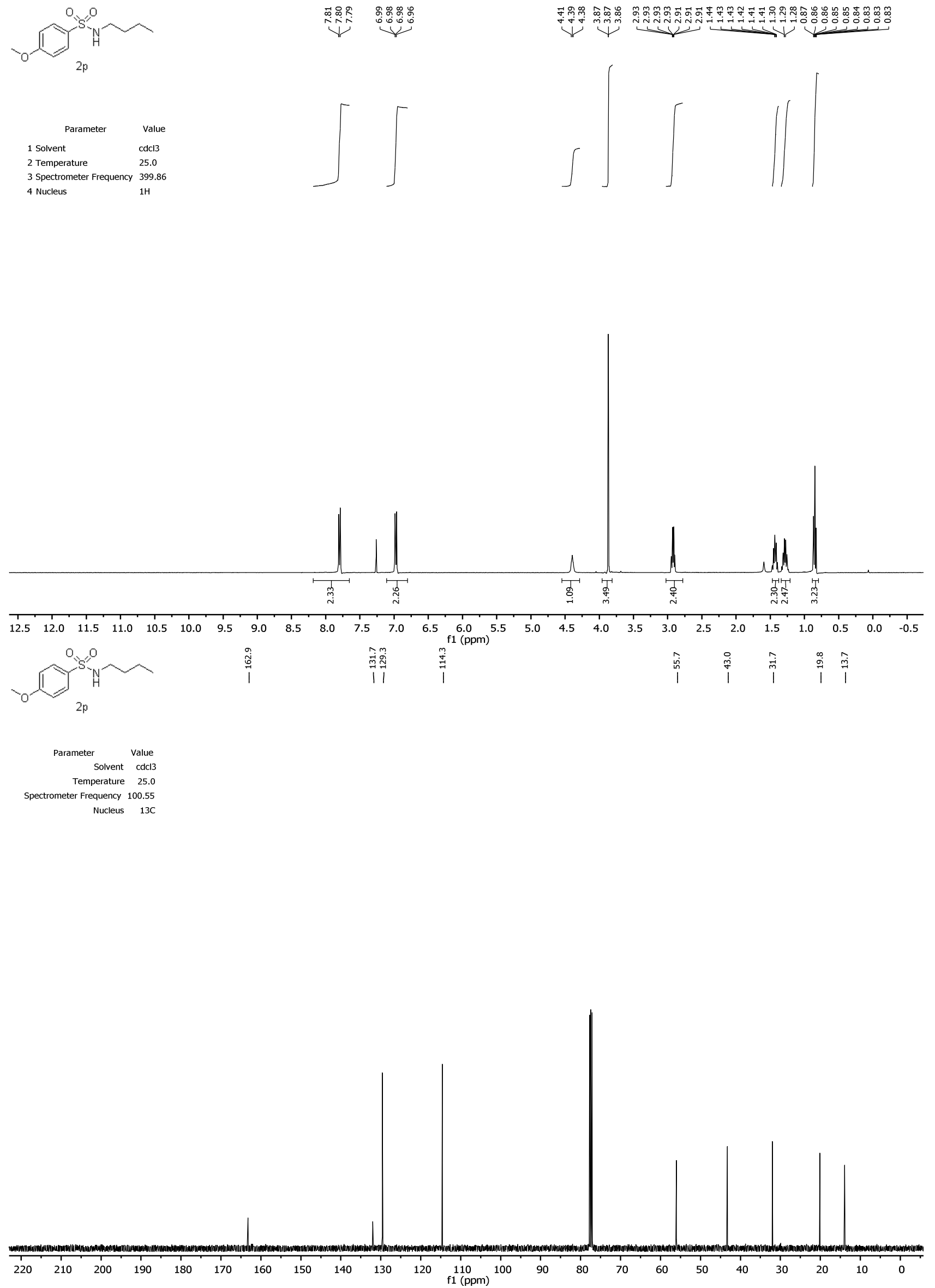
$N$-butyl-4-bromobenzenesulfonamide (2q)
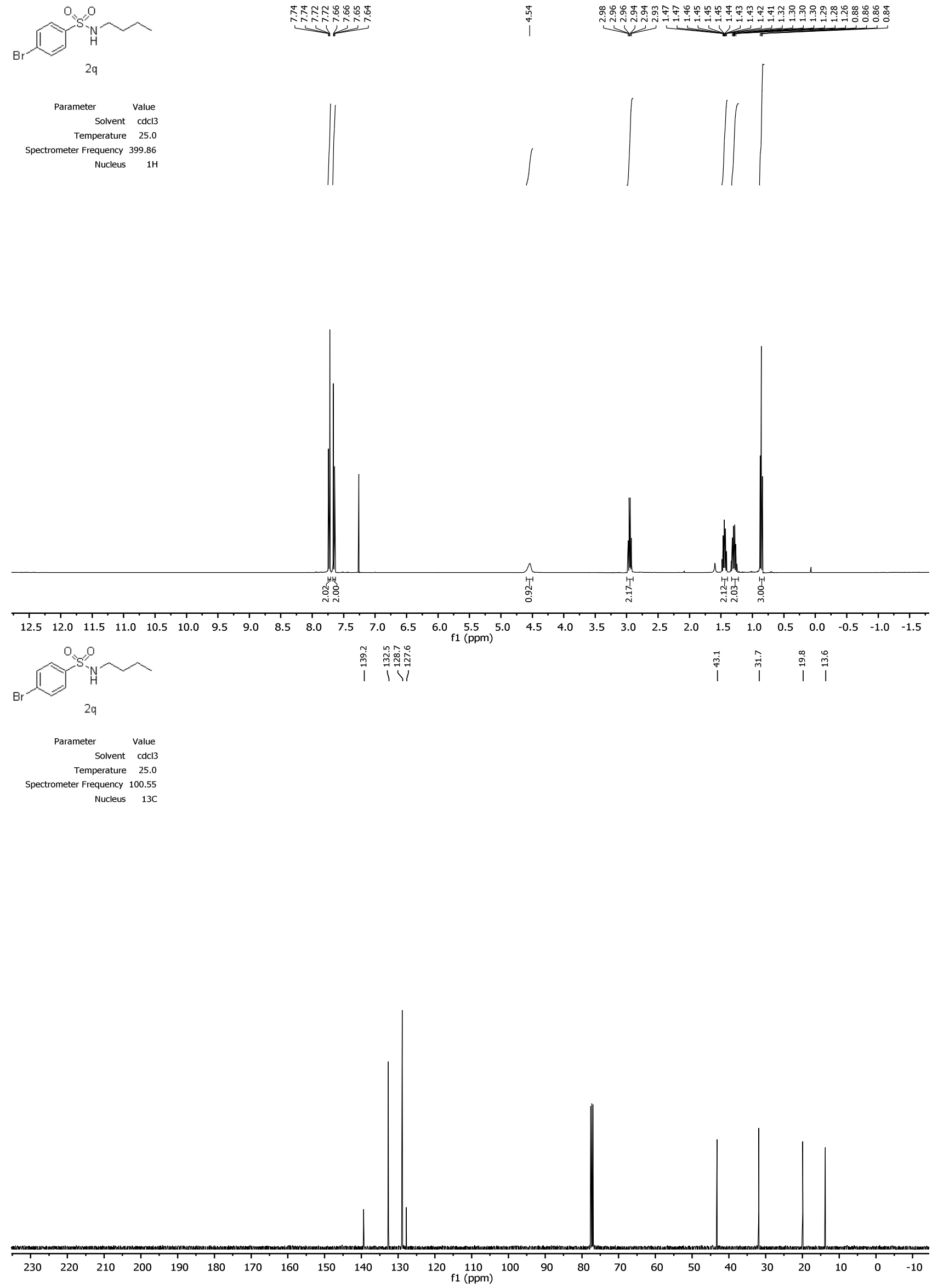
4-Acetyl- $N$-butylbenzenesulfonamide (2r), Table 2 prepared using standard conditions
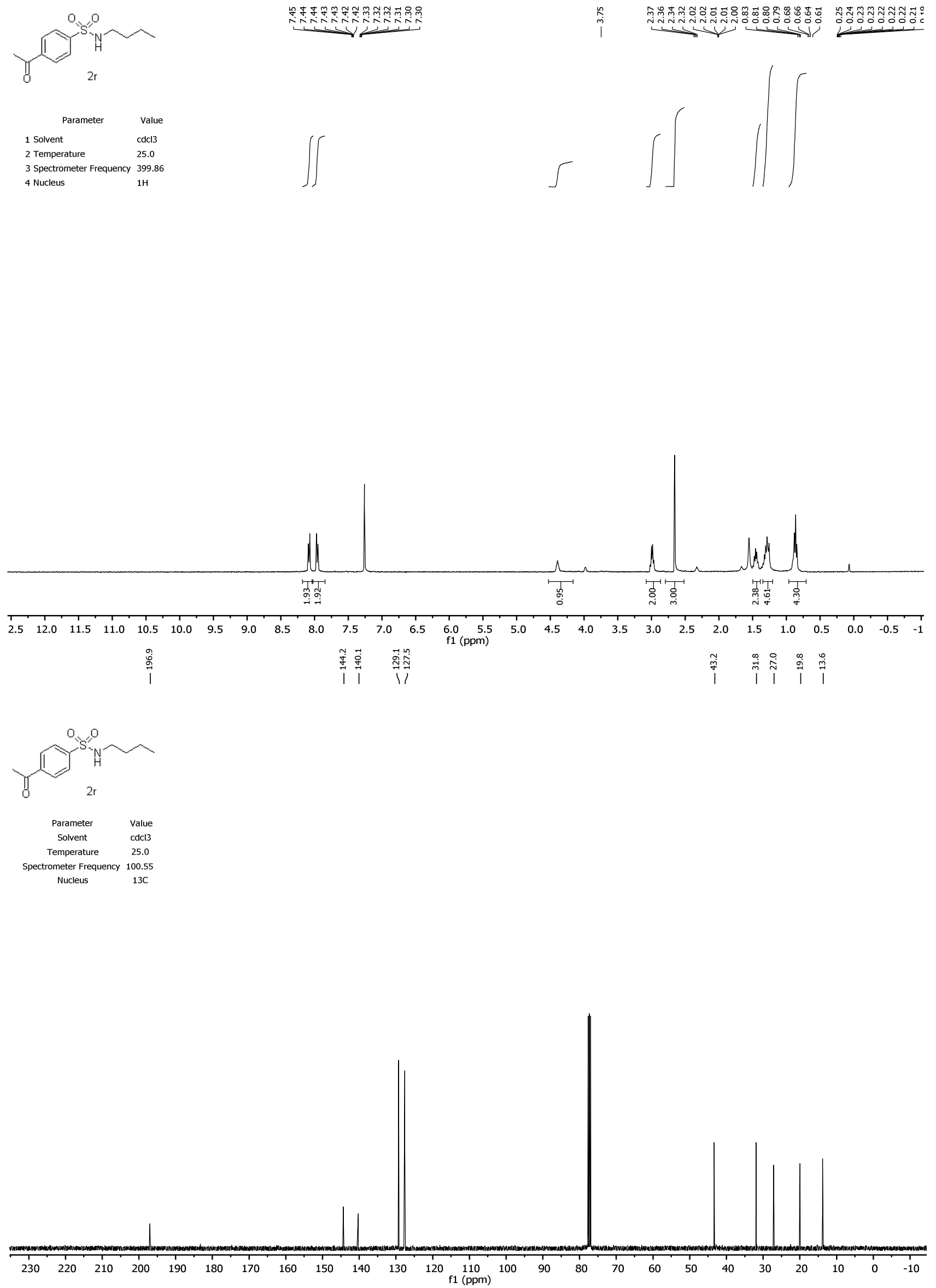
$N$-butyl-2-bromobenzenesulfonamide (2s)

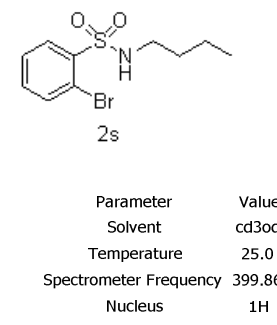

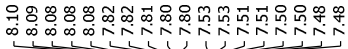

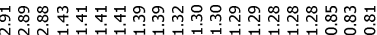

ن
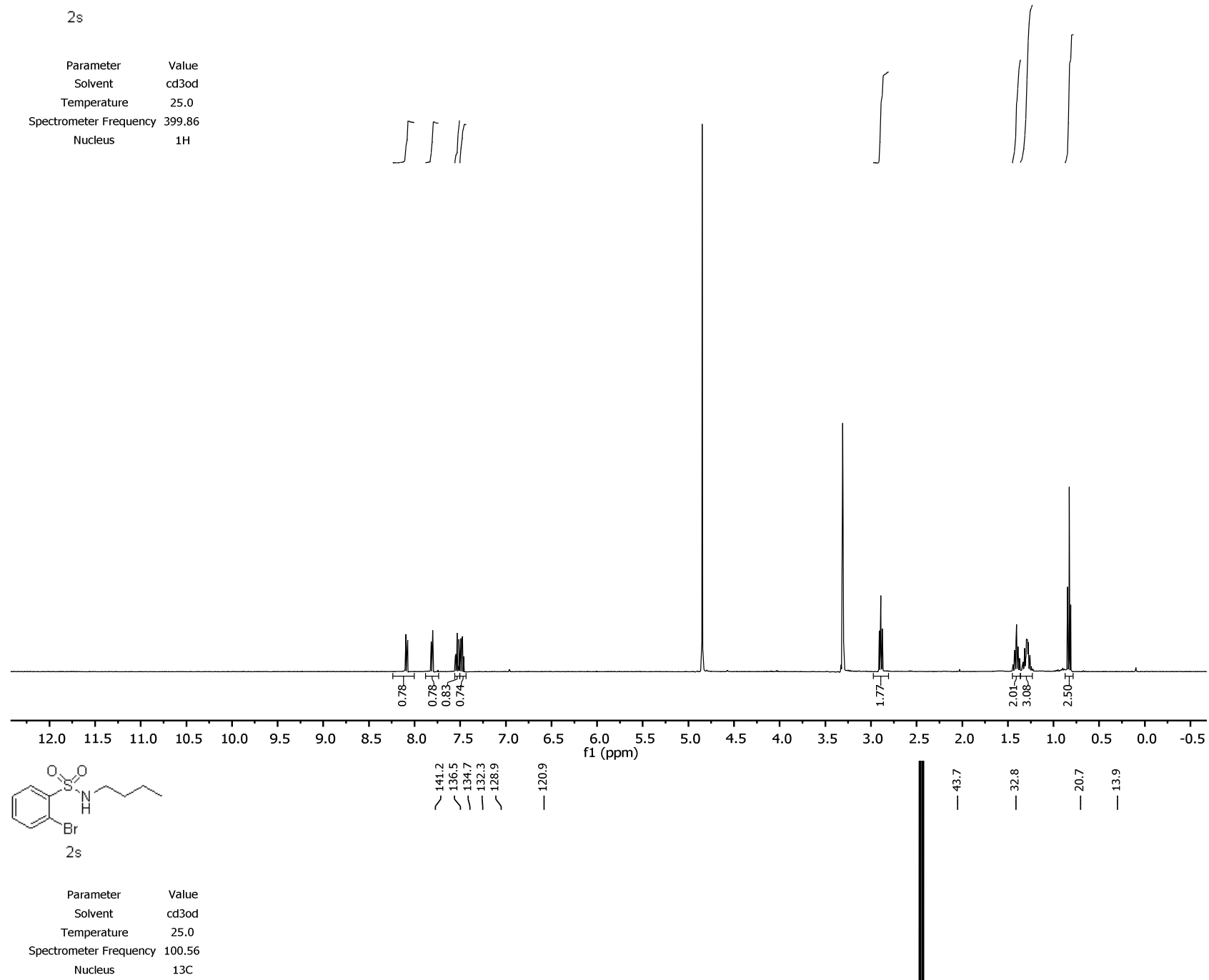

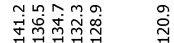

ता1र

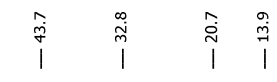

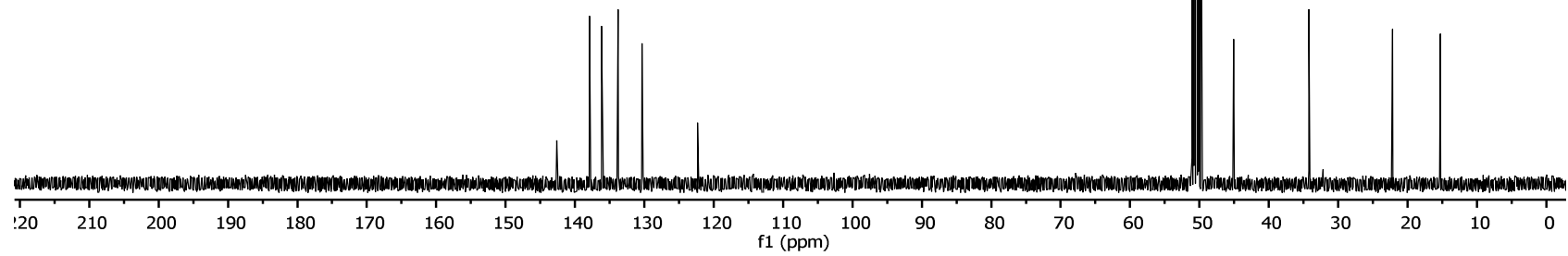


$N$-butylthiophene-2-sulfonamide (2v)
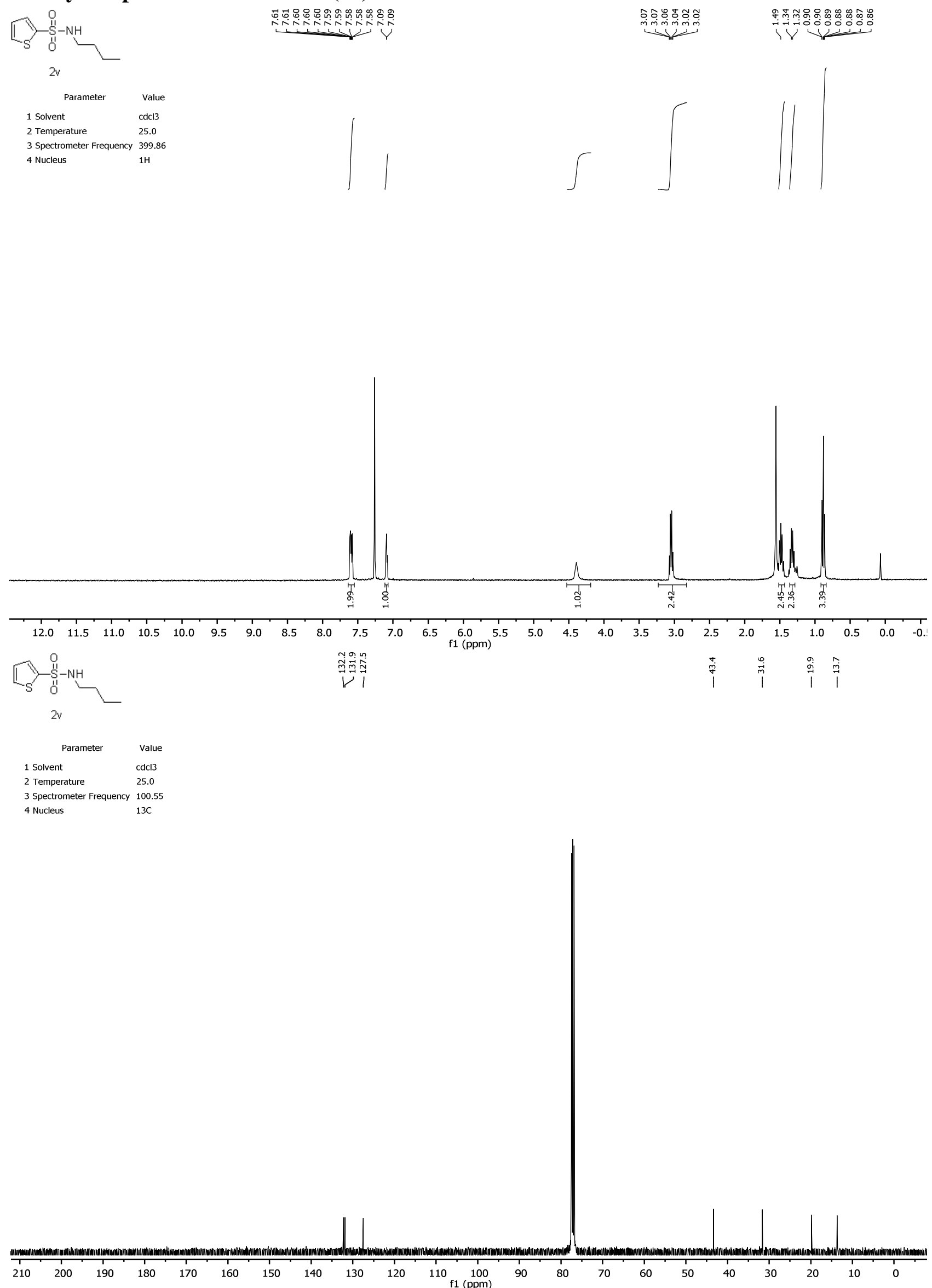
$\mathrm{N}$-butylbenzo[b]thiophene-3-sulfonamide (2w)
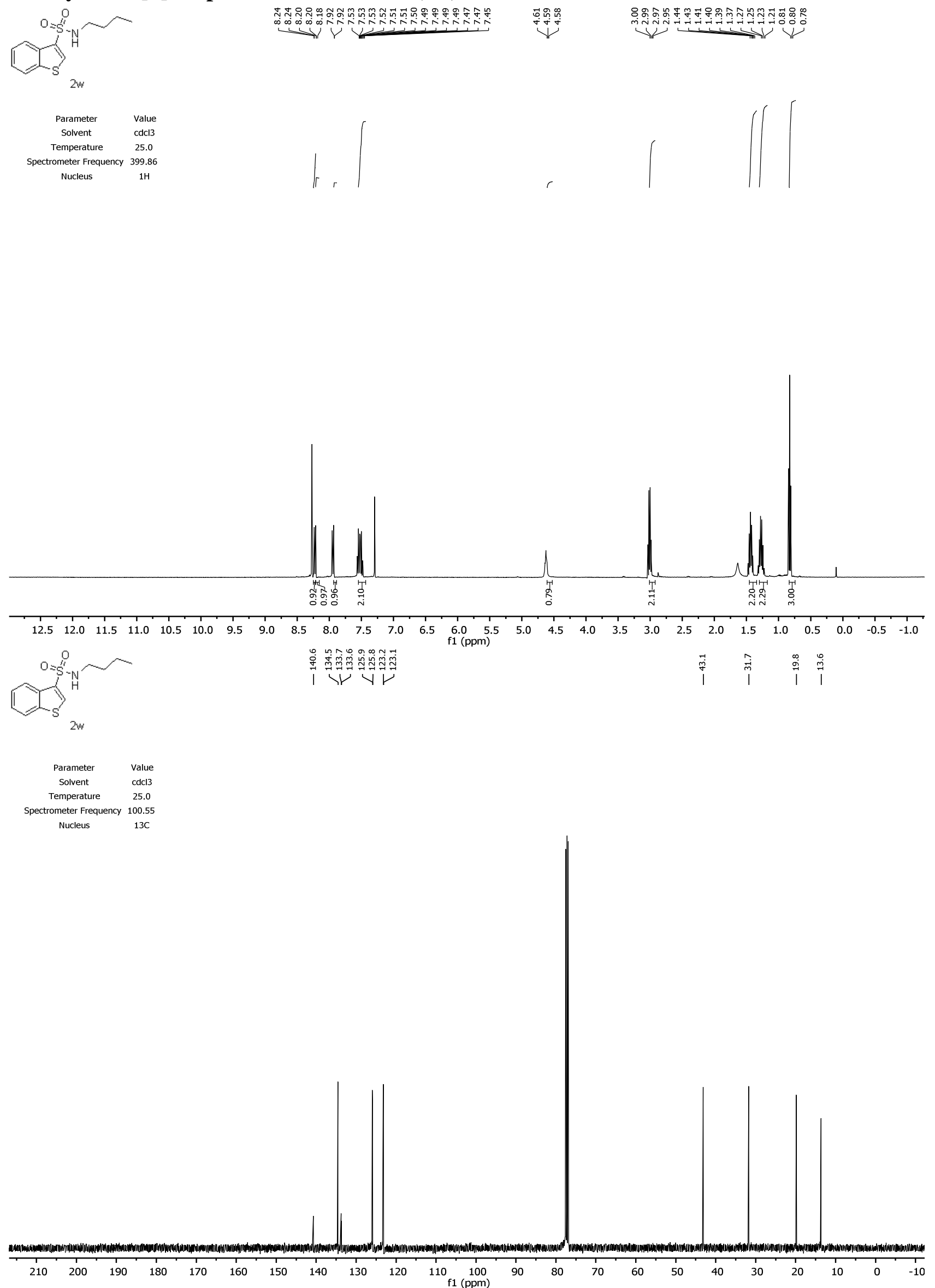


\section{5-(N-butylsulfamoyl)-4-chloro-2-((furan-2-ylmethyl)amino)benzoic acid (2x)}
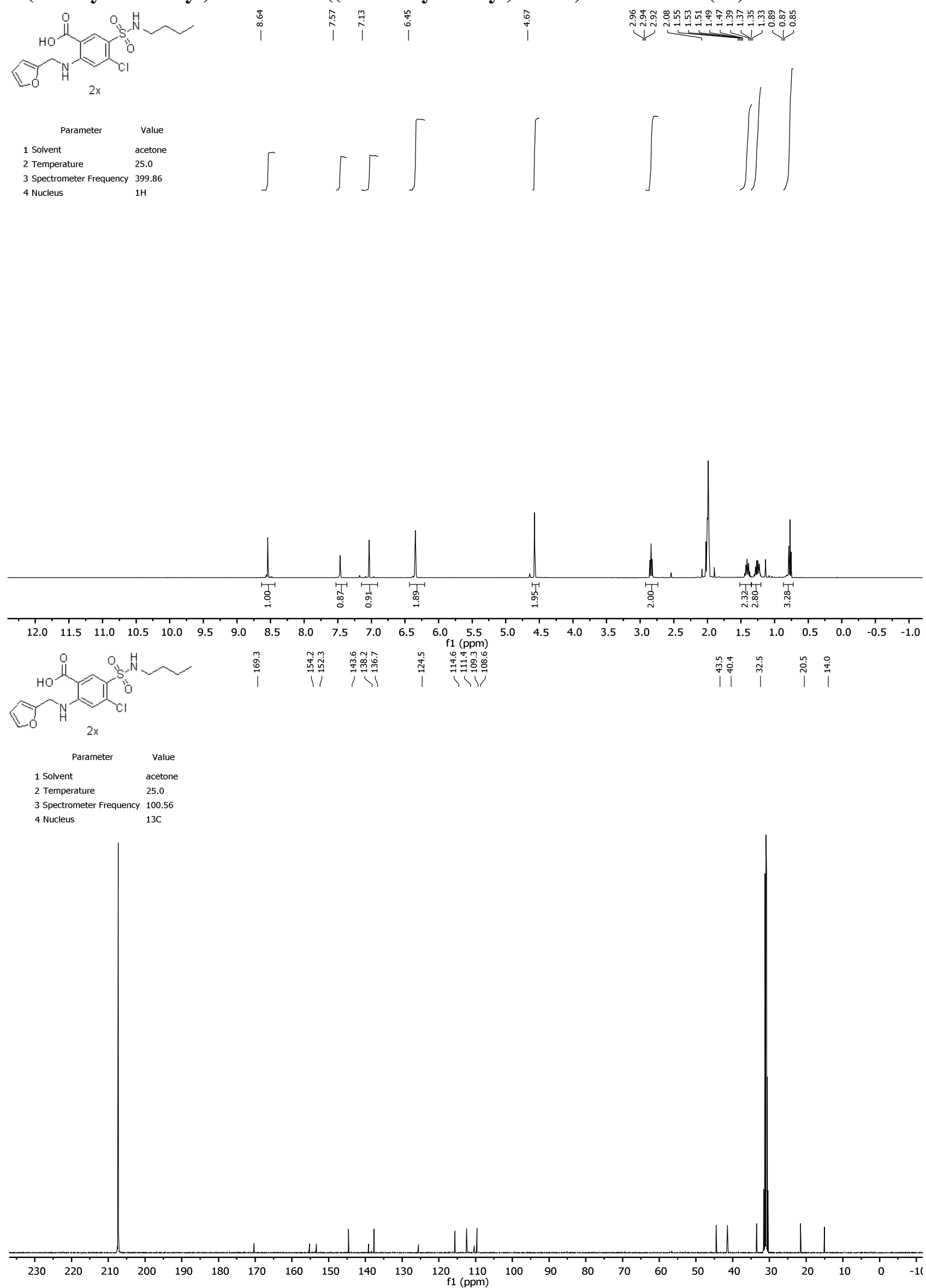
4-Methyl- $N$-(phenylcarbamoyl)benzenesulfonamide (Table 3, entry 3) (3a)

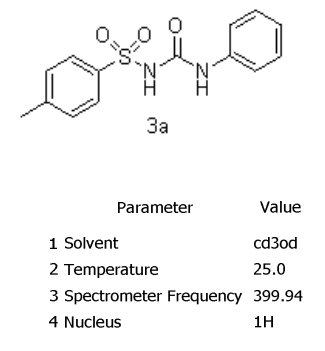

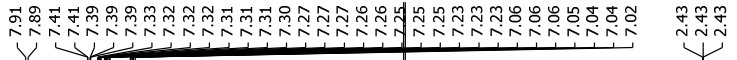
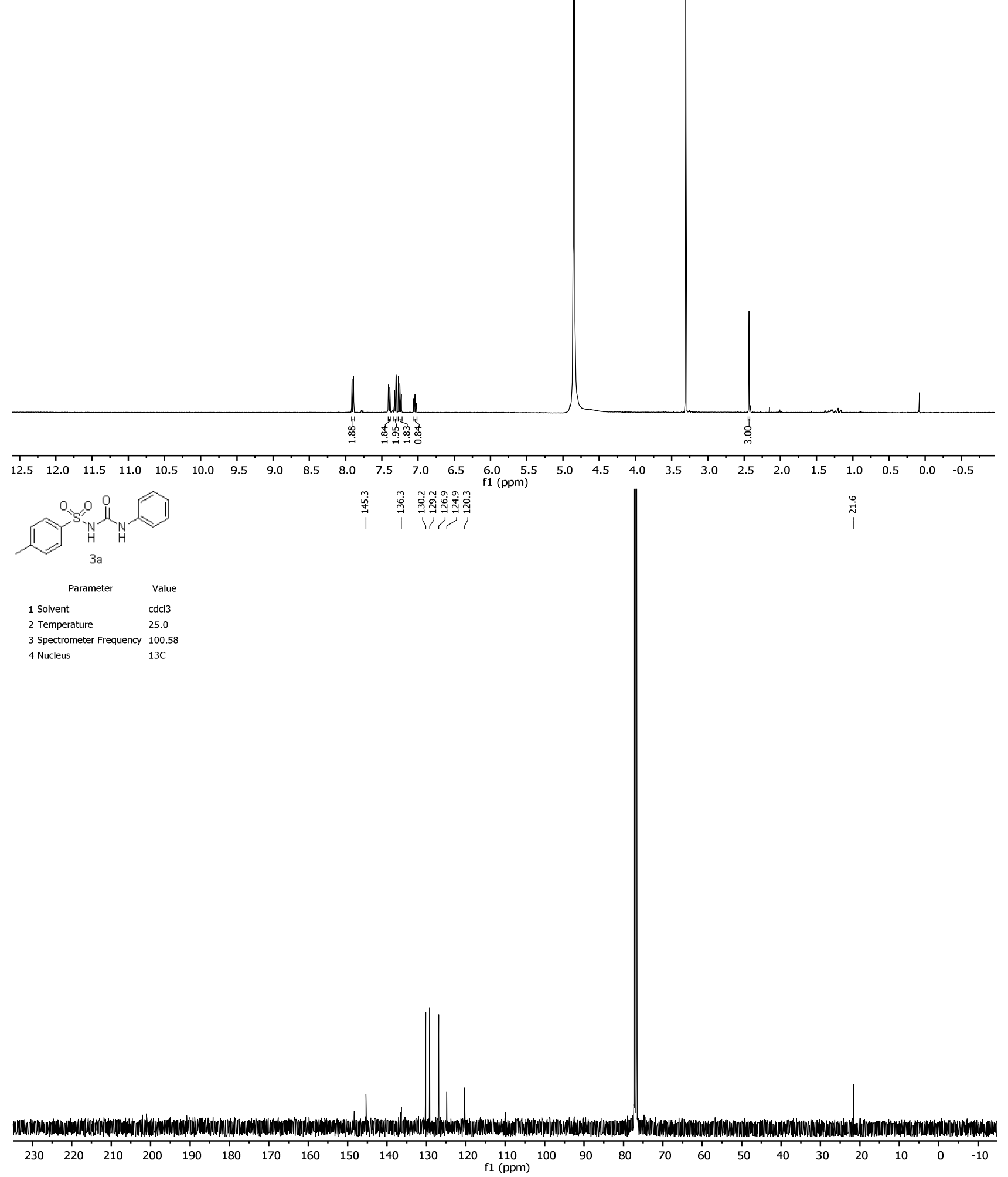
Triethylammonium $N$-((4-methoxyphenyl)carbamoyl)-4-methylbenzenesulfonamide (3b)
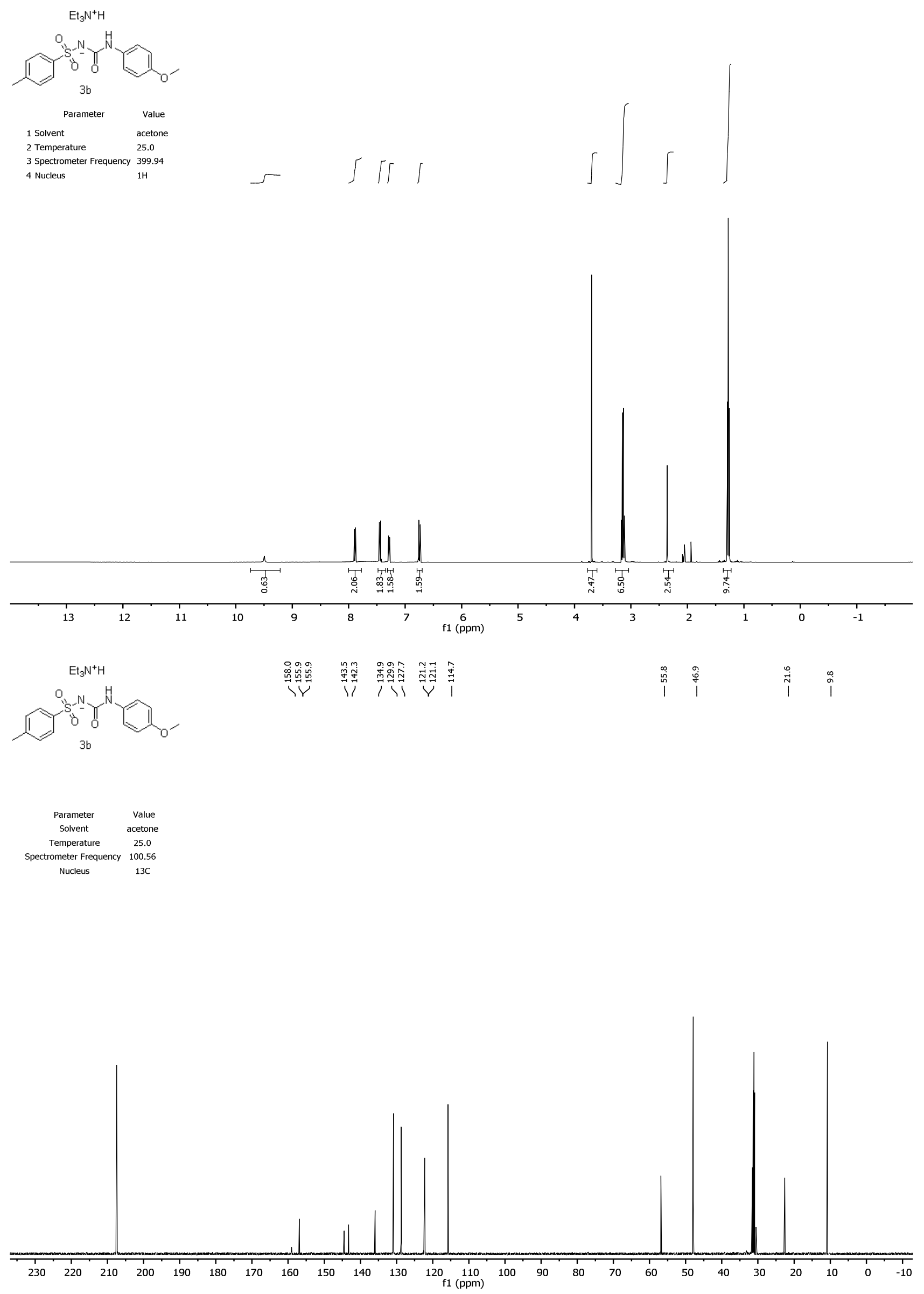
Triethylammonium $N$-((4-bromophenyl)carbamoyl)-4-methylbenzenesulfonamide (3c)
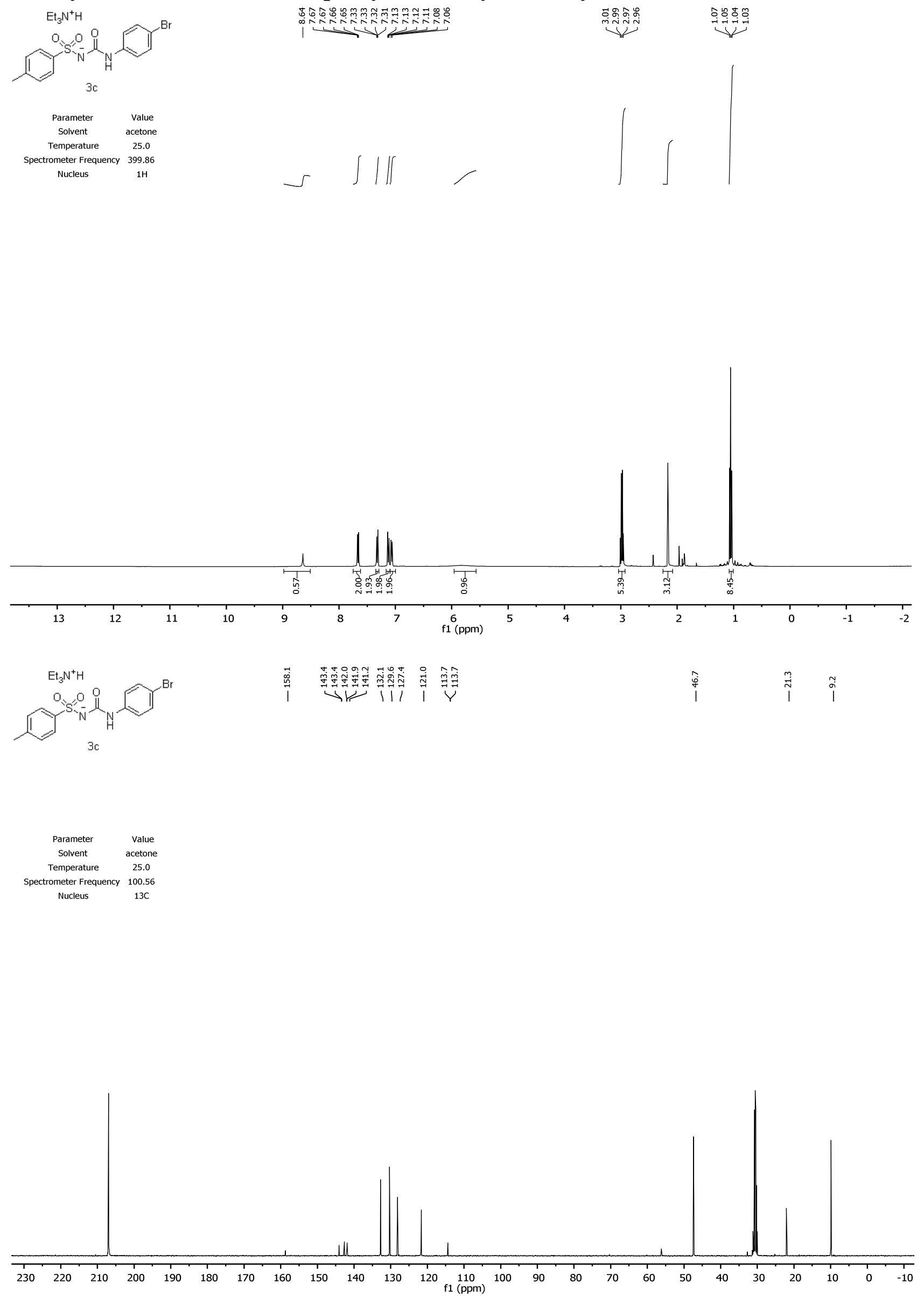
Triethylammonium $N$-((4-chlorophenyl)carbamoyl)-4-methylbenzenesulfonamide (3d)
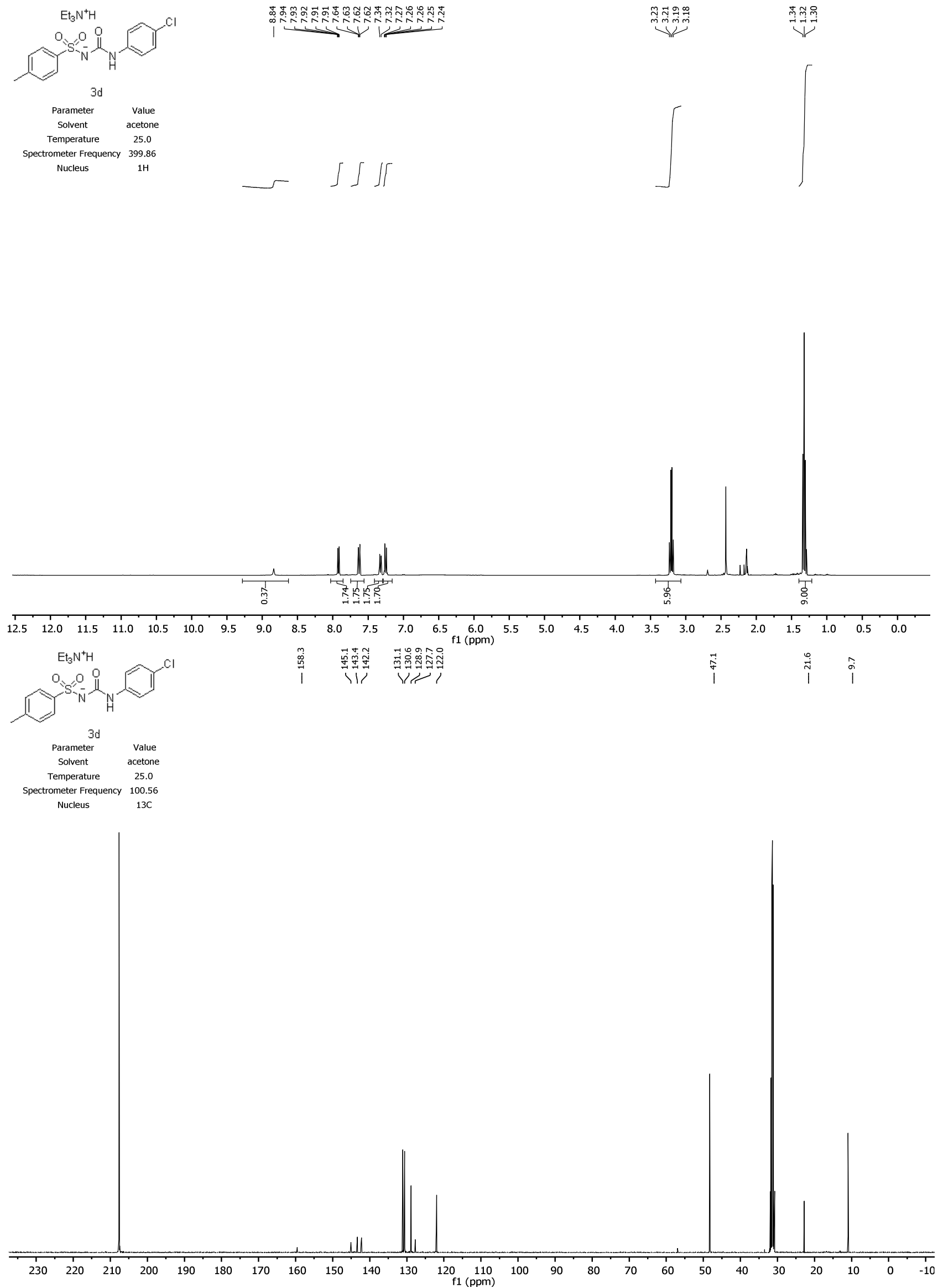
Triethylammonium $N$-((4-cyanophenyl)carbamoyl)-4-methylbenzenesulfonamide (3e)
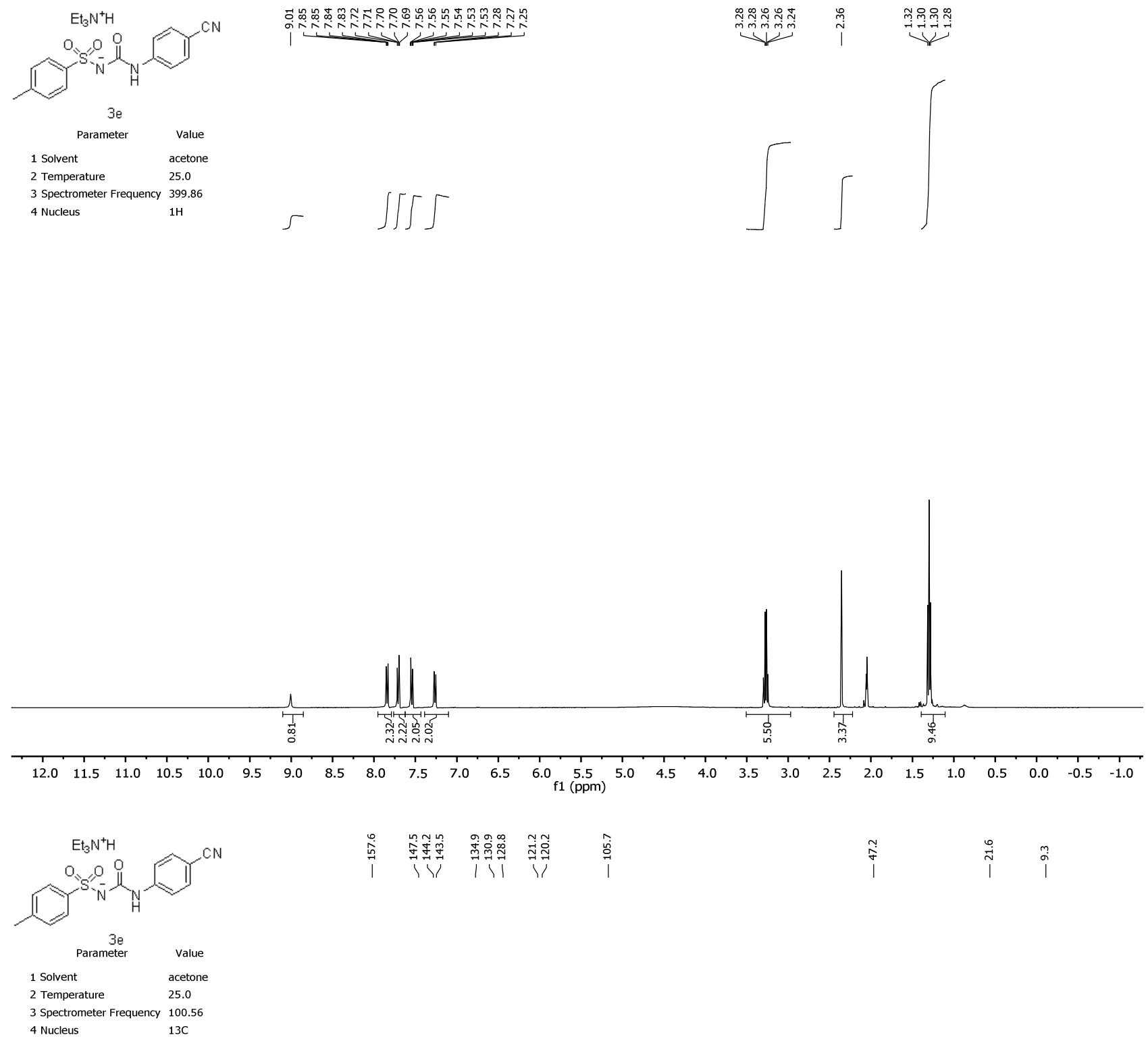

\section{|}

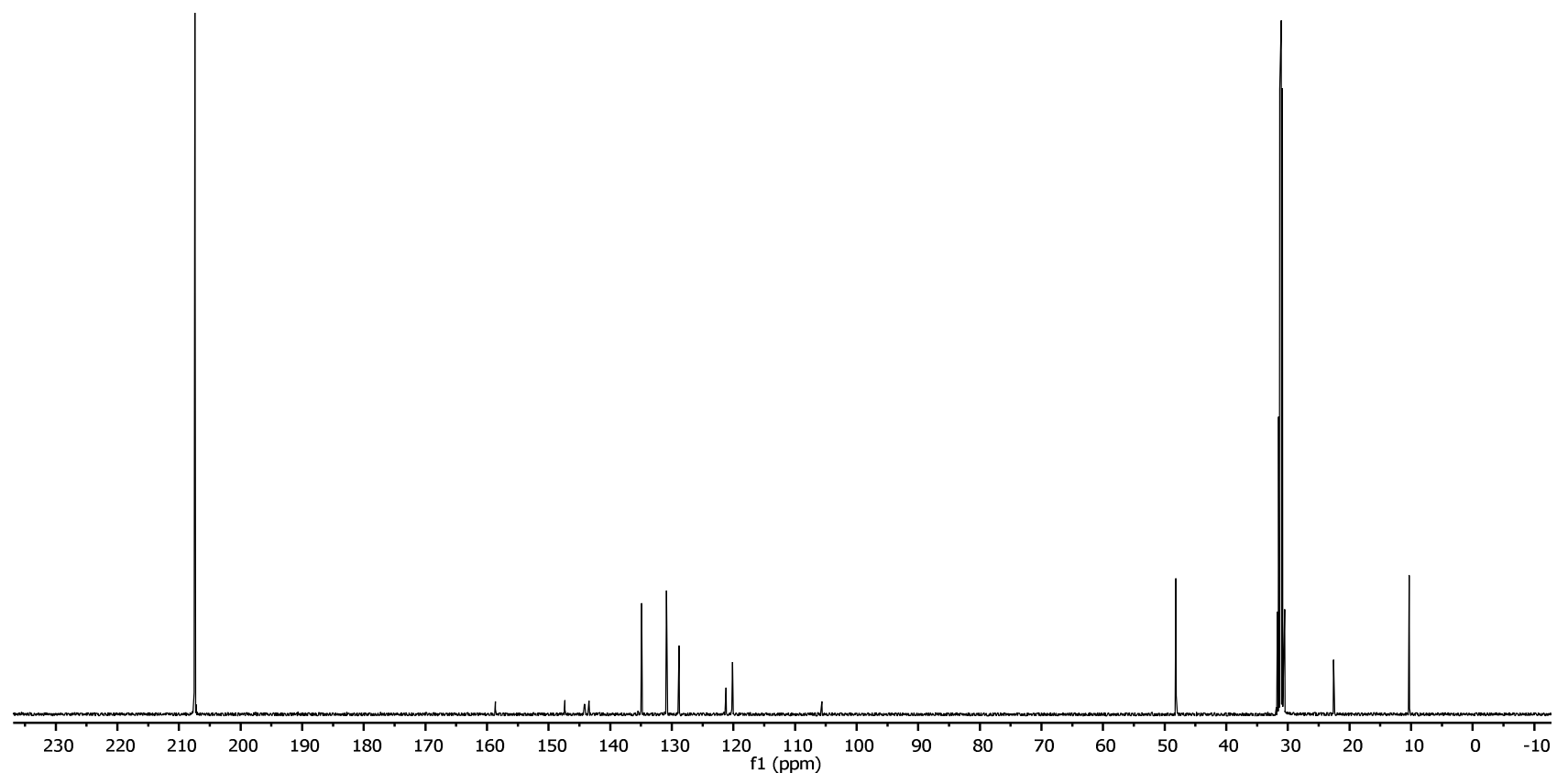


Triethylammonium methyl 4-(3-tosylureido)benzoate (3f)
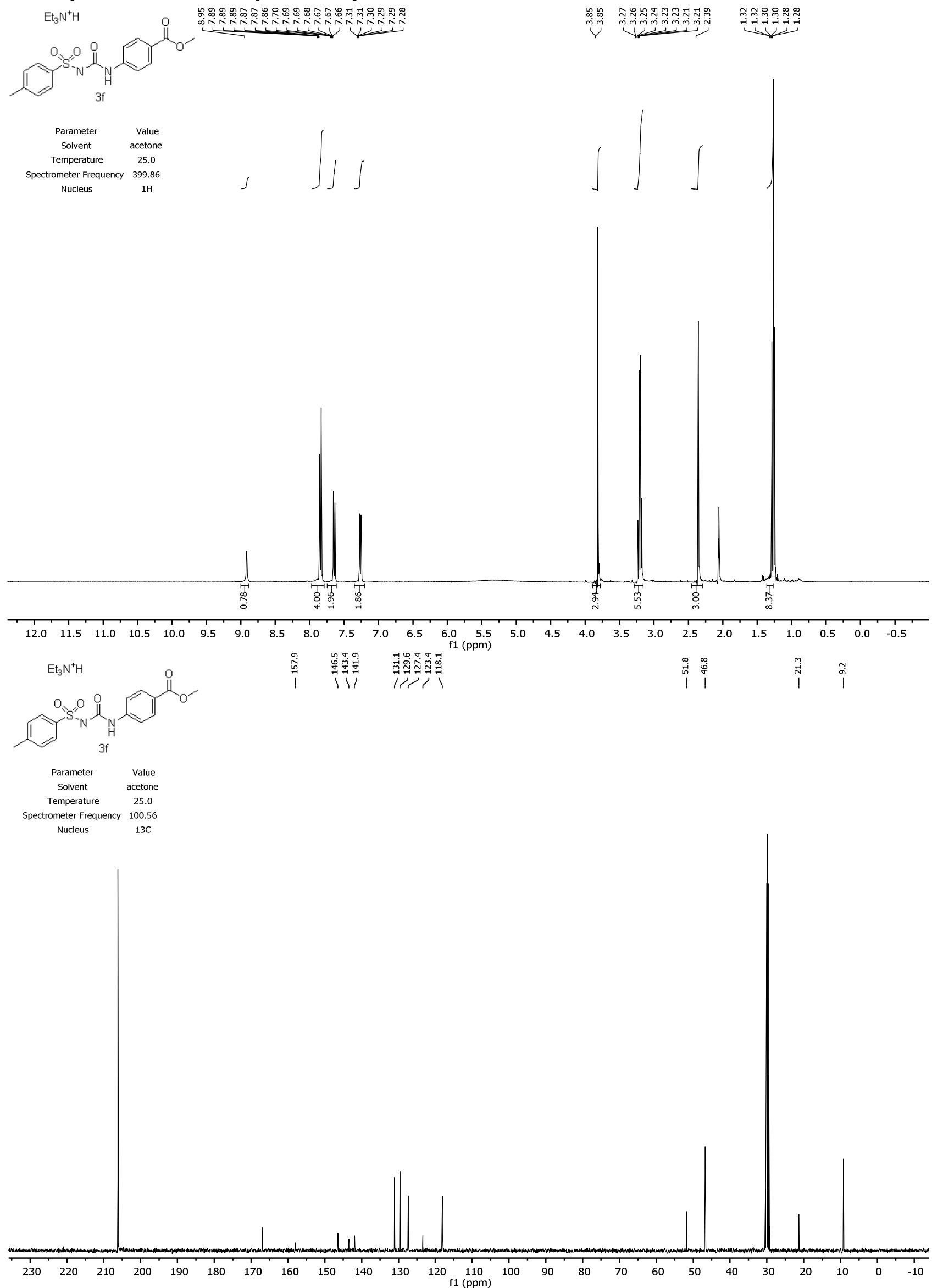
Triethylammonium 4-methyl- $N$-(o-tolylcarbamoyl)benzenesulfonamide (3g)
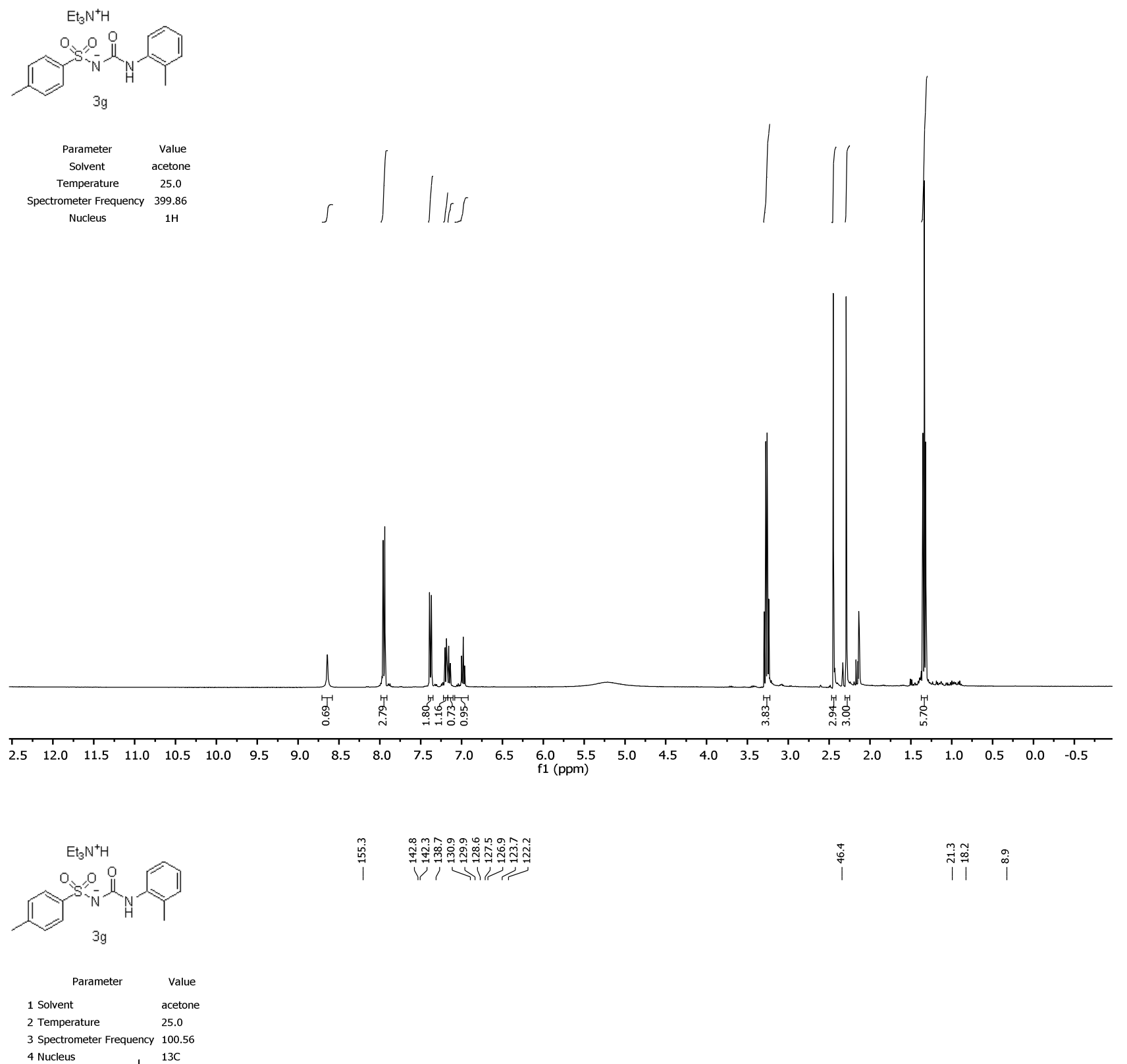

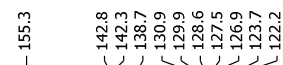

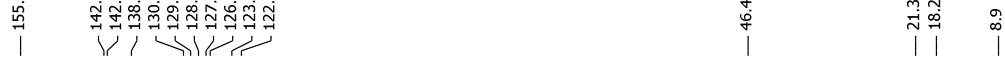

3 Spectrometer Frequency 100.56

\begin{tabular}{l|l}
4 Nucleus & $13 \mathrm{C}$
\end{tabular}

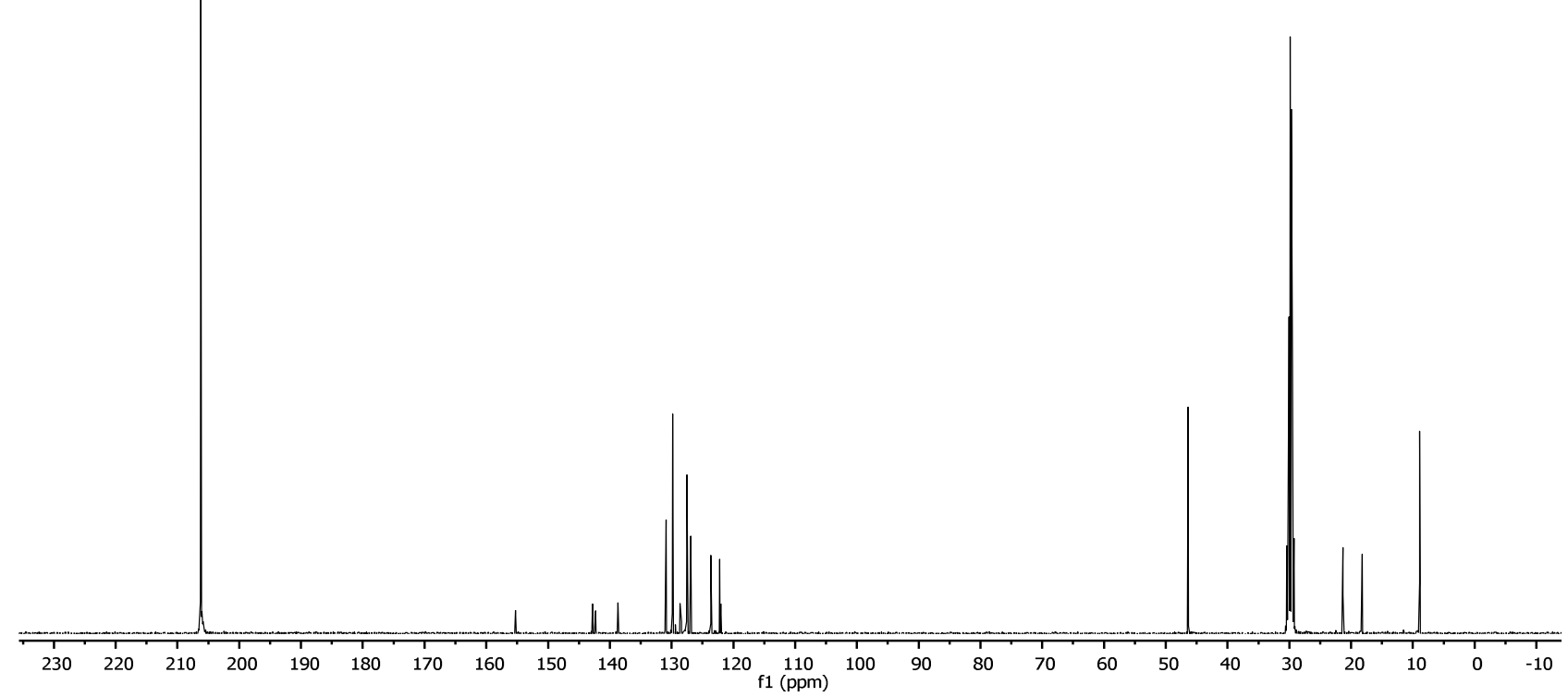


Triethylammonium $N$-((2-chlorophenyl)carbamoyl)-4-methylbenzenesulfonamide (3h)
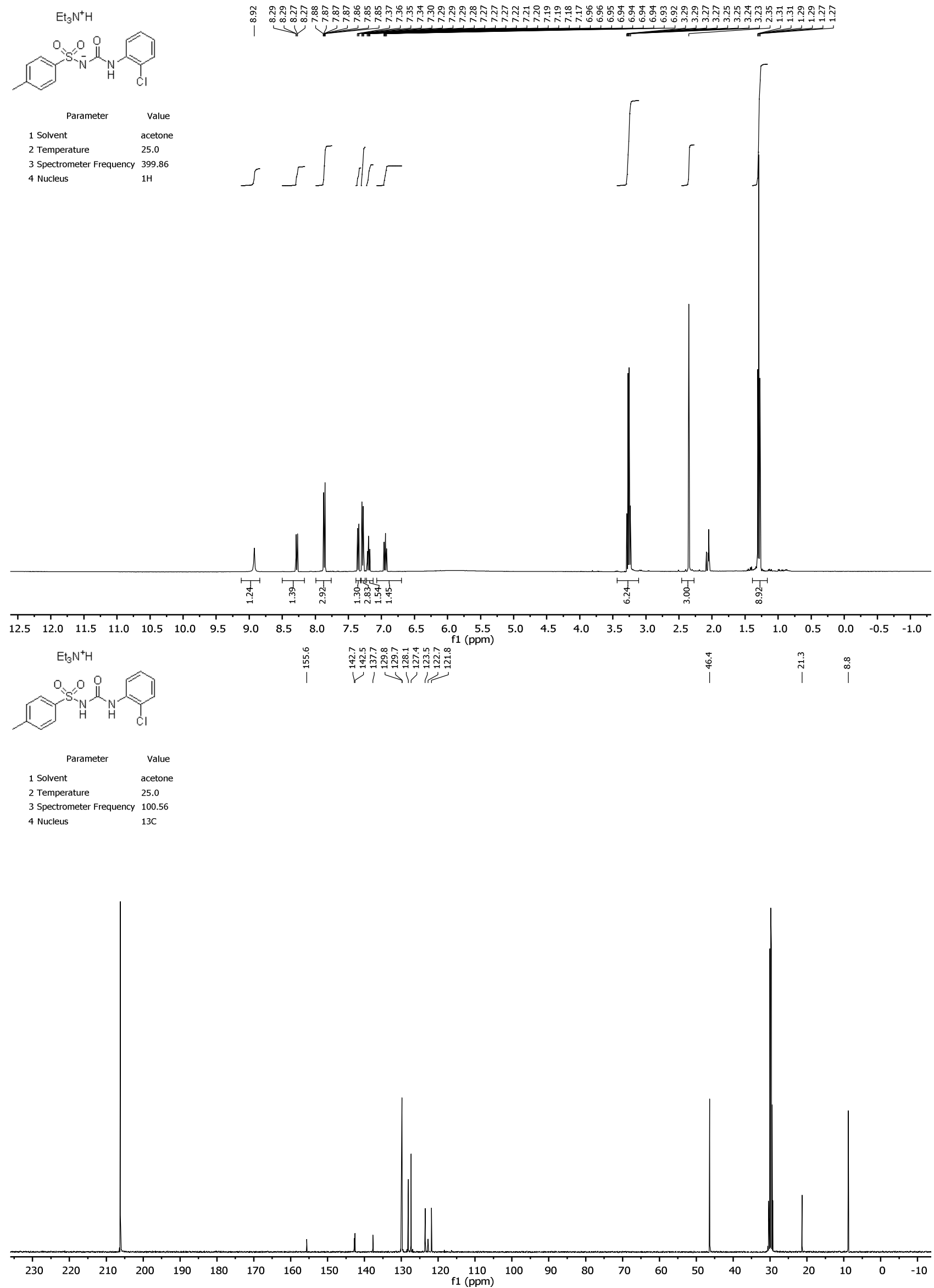
Triethylammonium 4-Methoxy- $N$-(phenylcarbamoyl)benzenesulfonamide (3i)
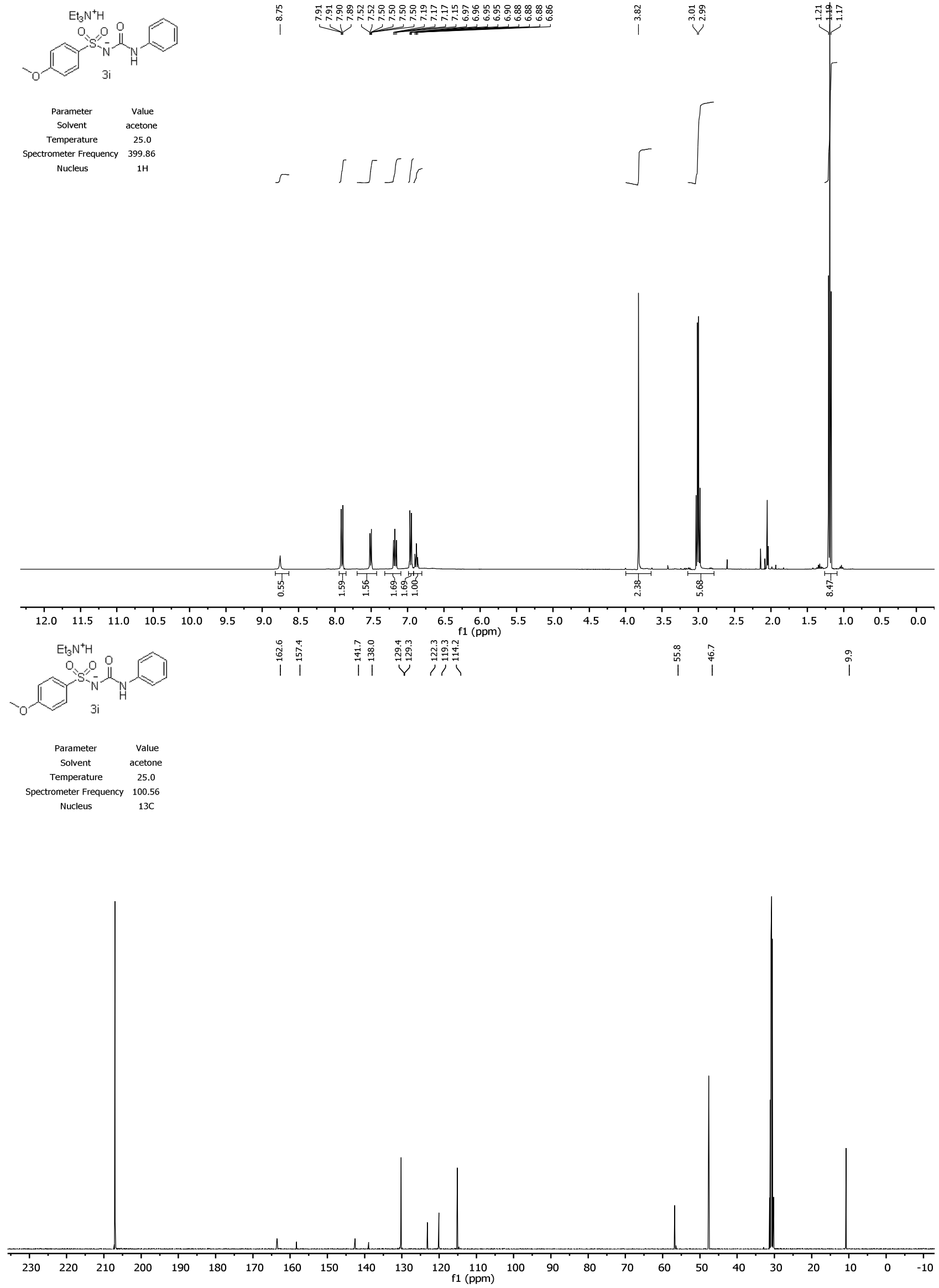
Triethylammonium 4-Acetyl- $N$-(phenylcarbamoyl)benzenesulfonamide (3j)
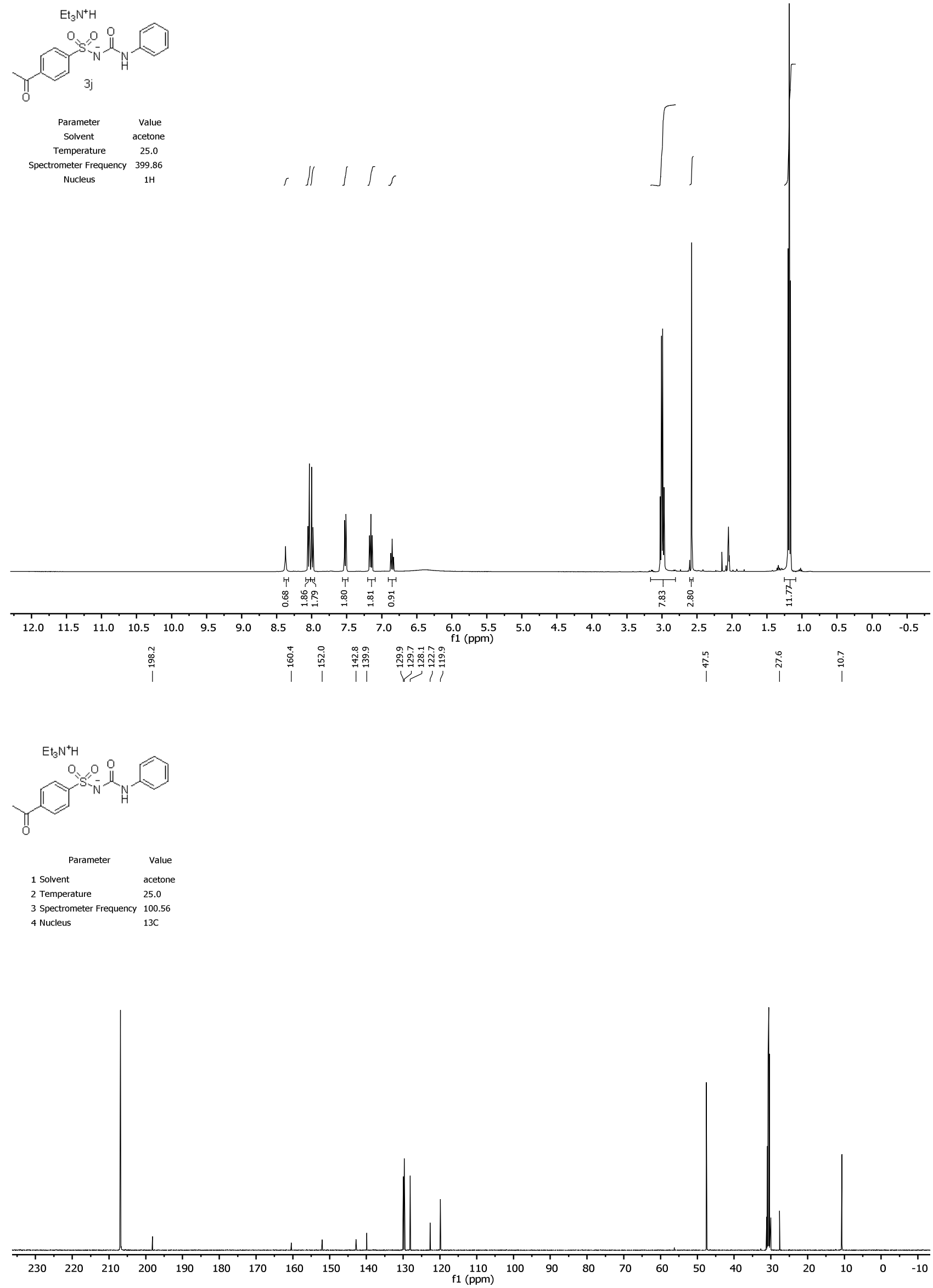
5-Chloro- $N$-(phenylcarbamoyl)thiophene-2-sulfonamide (3k)
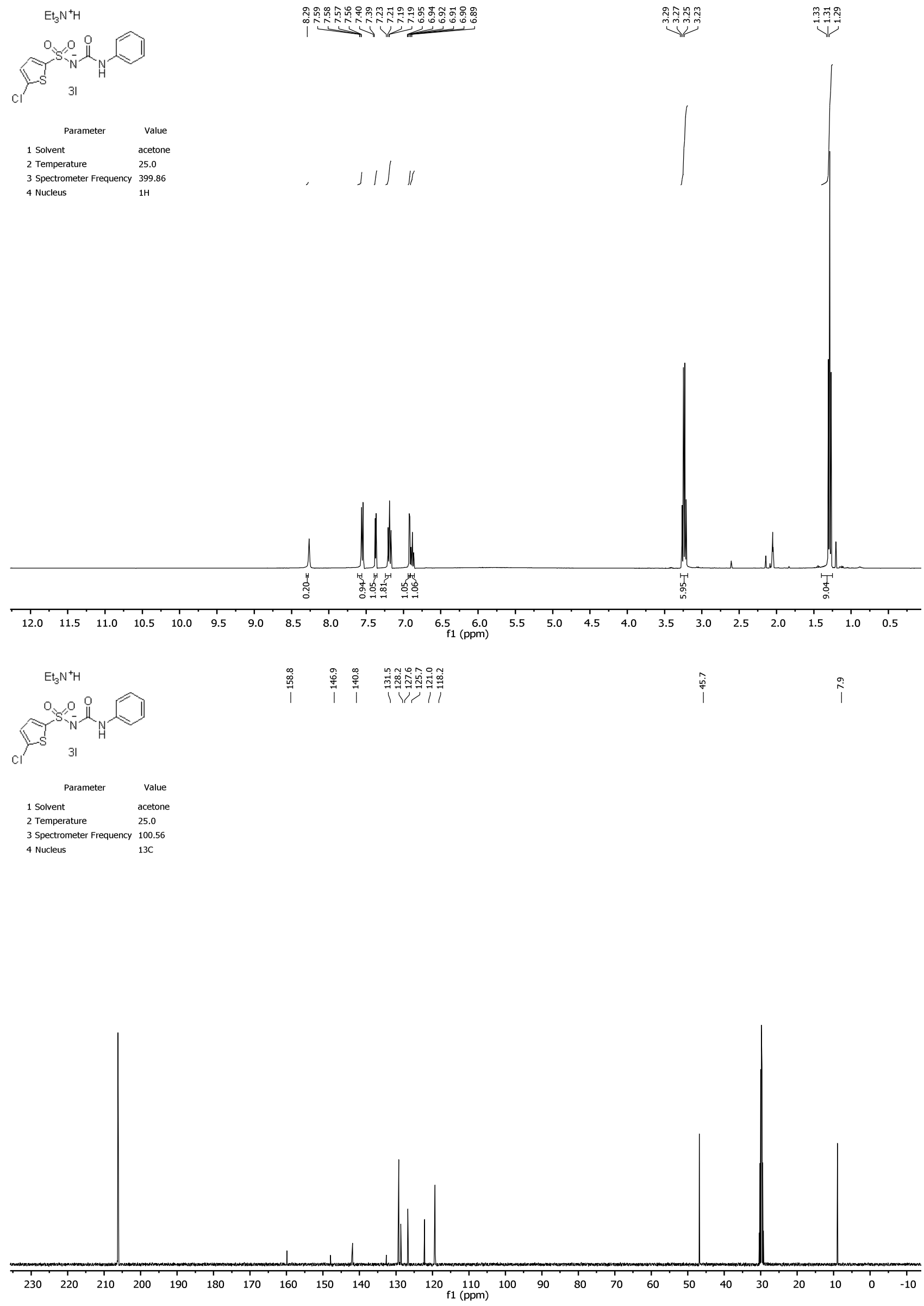
Triethylammonium $N$-(Phenylcarbamoyl)benzo[b]thiophene-3-sulfonamide (31)
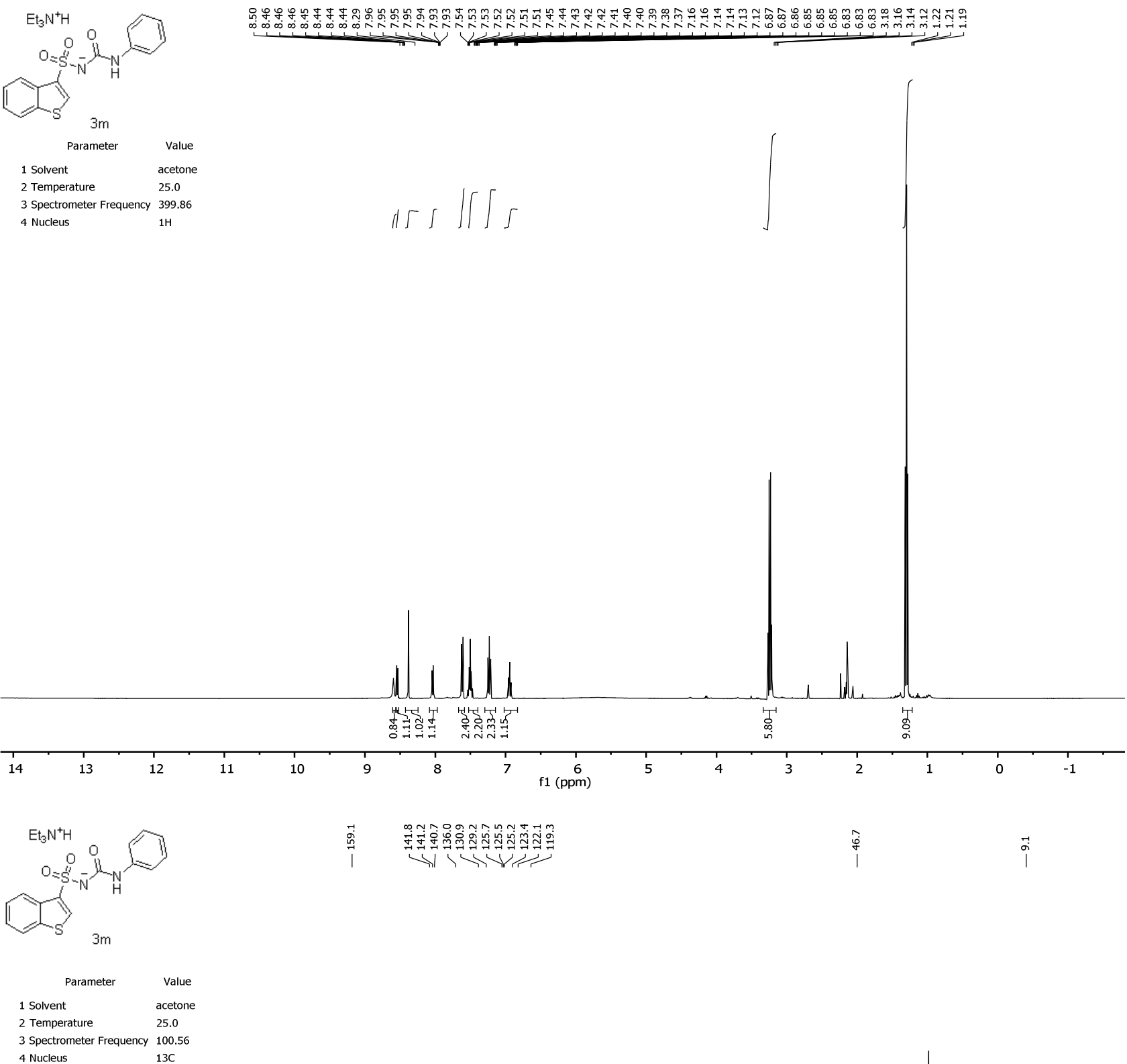

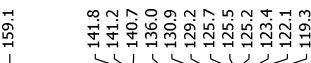

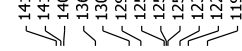

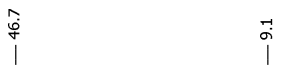

4 Nucleus $\quad 13 \mathrm{C}$

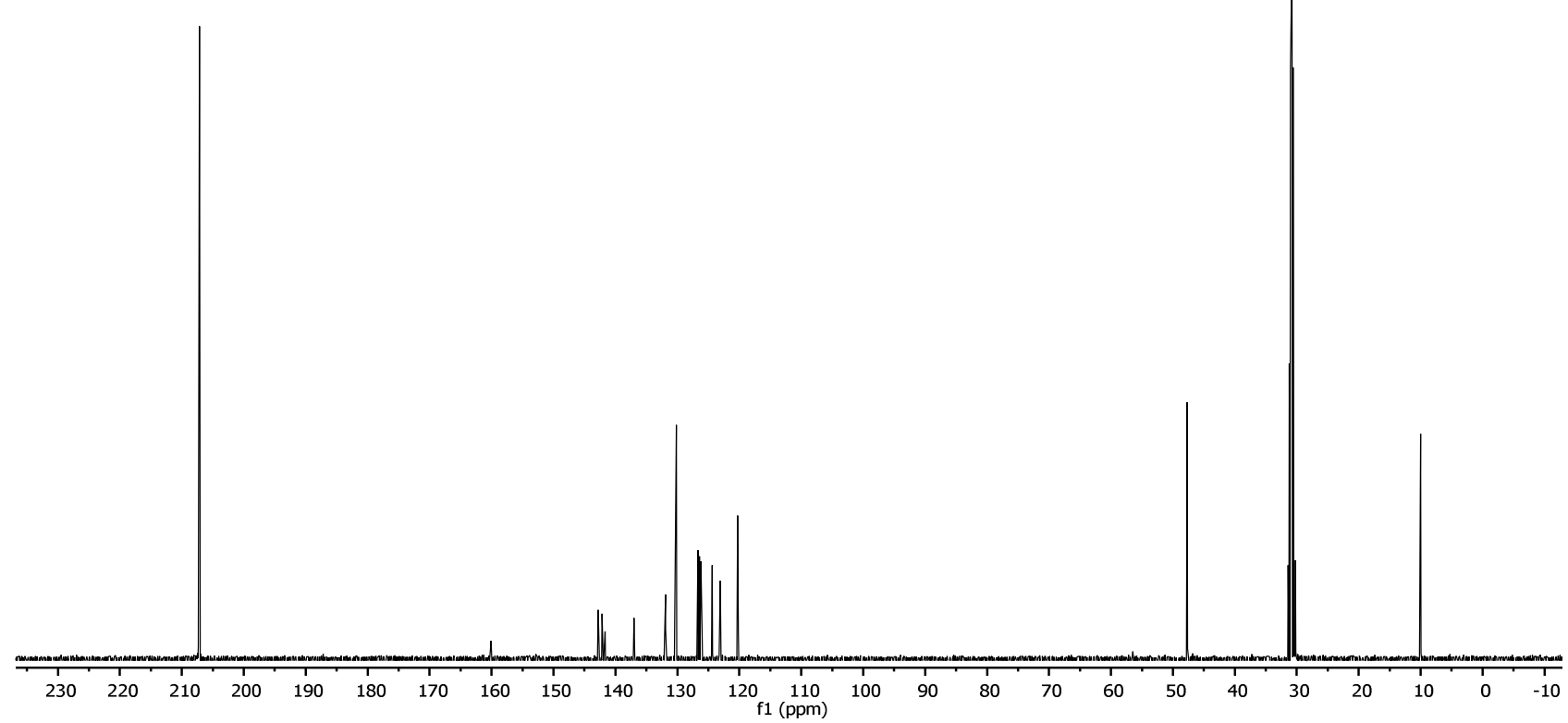


Butyl toluenesulfonylcarbamate (Table 5, entry 3) (4a)
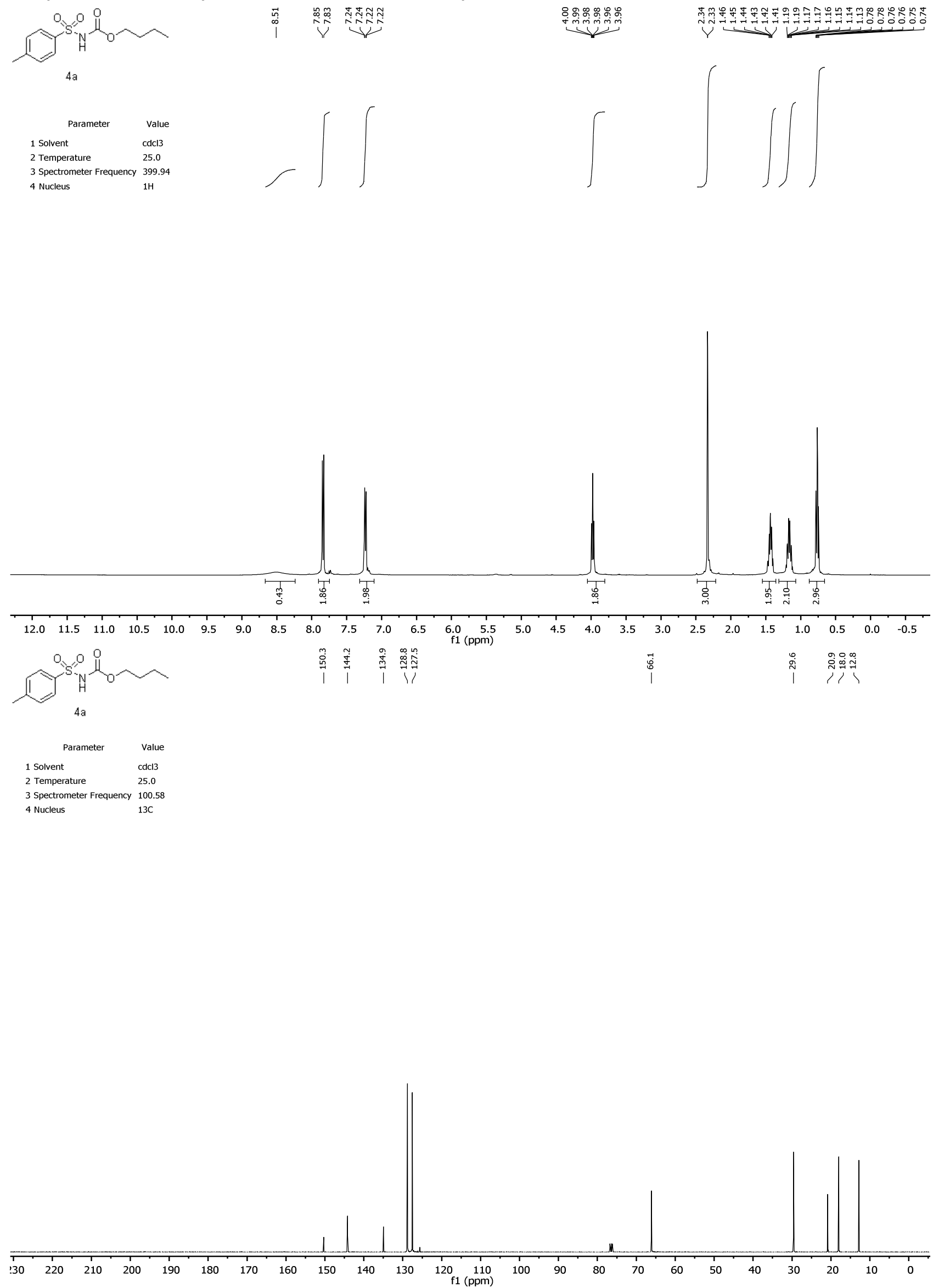
Methyl toluenesulfonylcarbamate (4b)
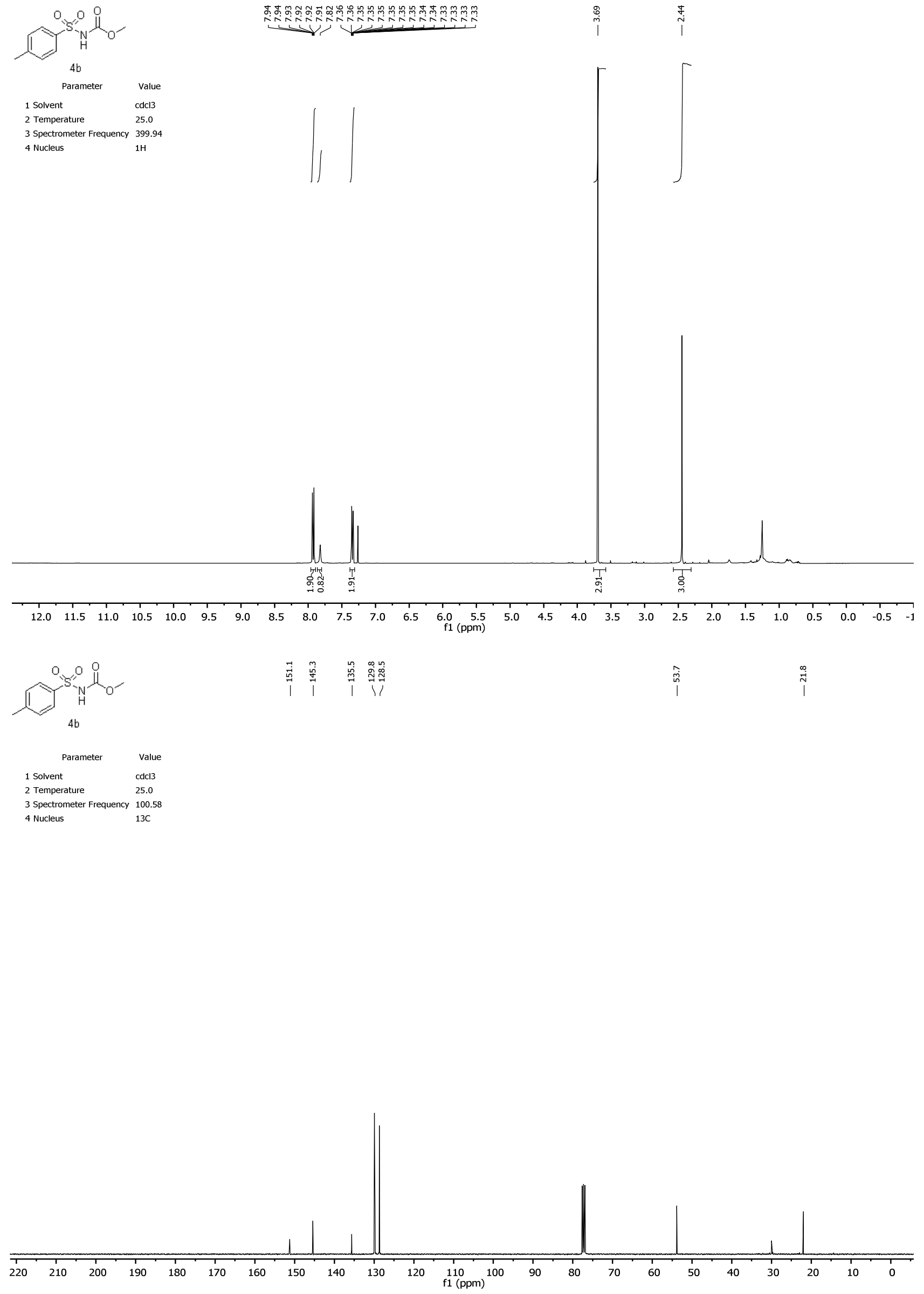
Isopropyl toluenesulfonylcarbamate (4c)
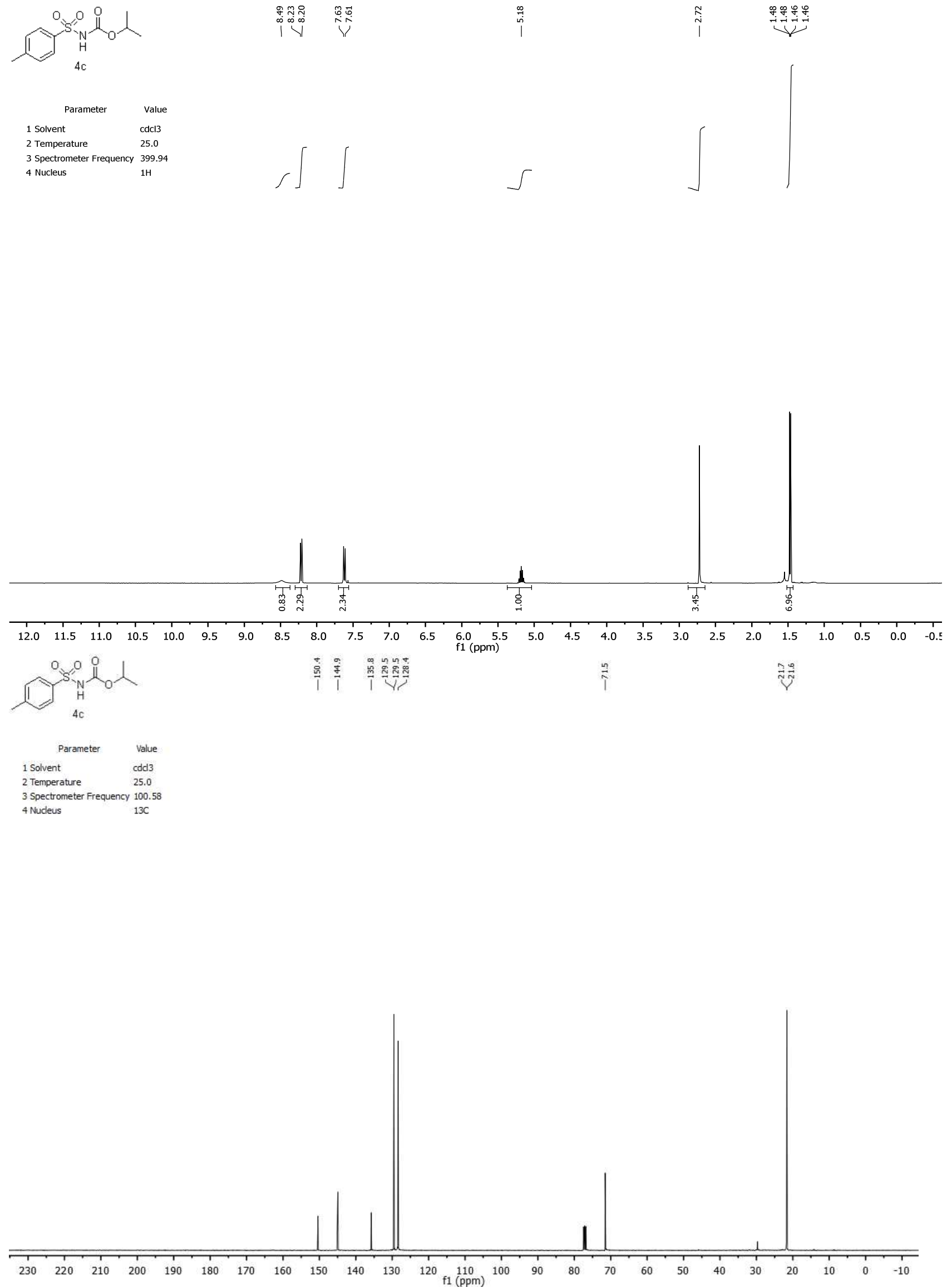
tert-Butyl toluenesulfonylcarbamate (4d)

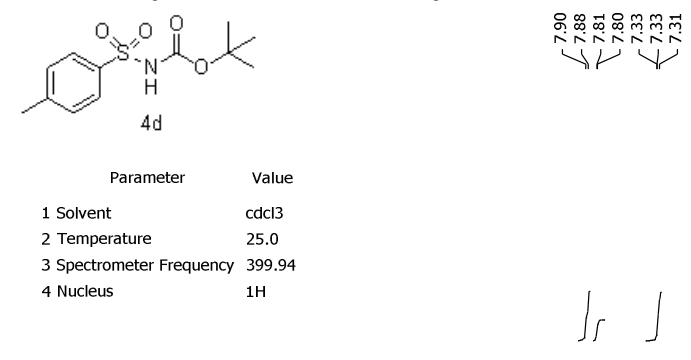

谒

Is

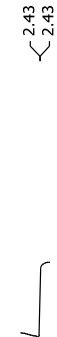

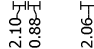

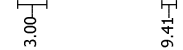

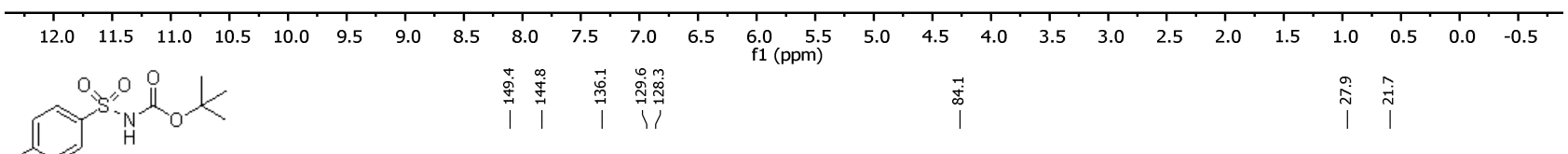

40

\begin{tabular}{ll}
\multicolumn{1}{c}{ Parameter } & Value \\
1 Solvent & cdc13 \\
2 Temperature & 25.0 \\
3 Spectrometer Frequency & 100.58 \\
4 Nucleus & $13 \mathrm{C}$
\end{tabular}

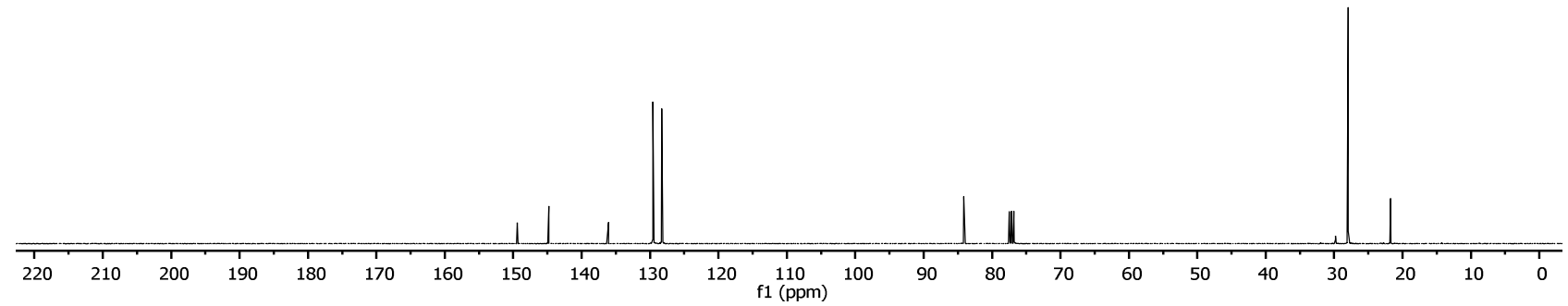




\section{Cyclohexyl toluenesulfonylcarbamate (4e)}

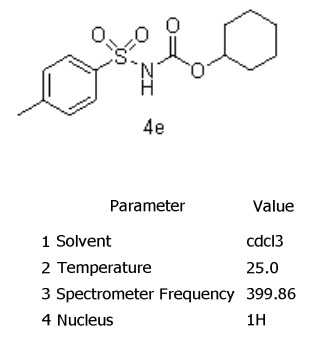

25.0

4 Nucleus $\quad 1 \mathrm{H}$

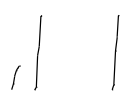

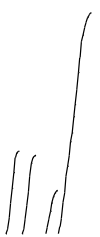
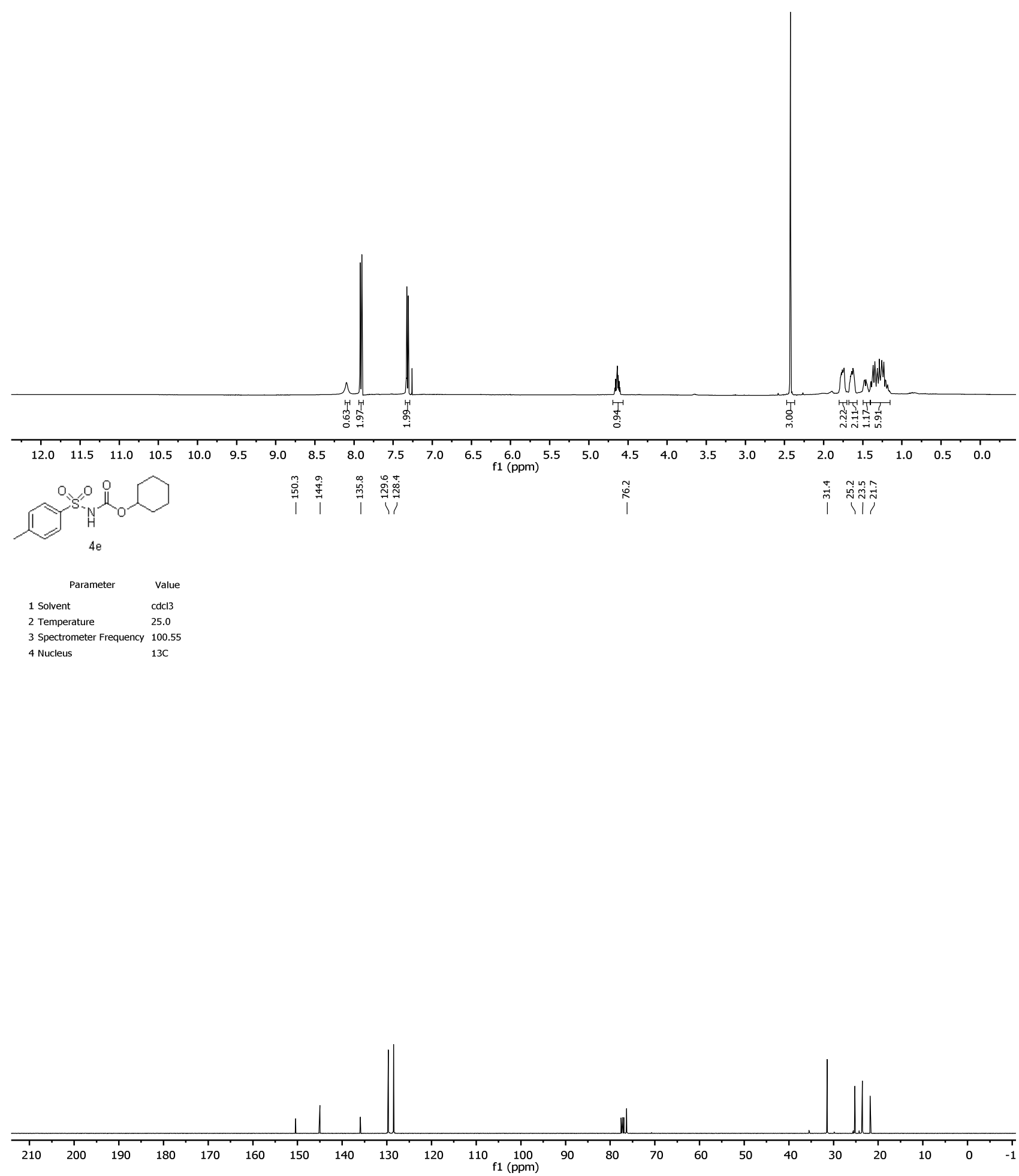
4,4,4-trifluorobutyl toluenesulfonylcarbamate (4f)
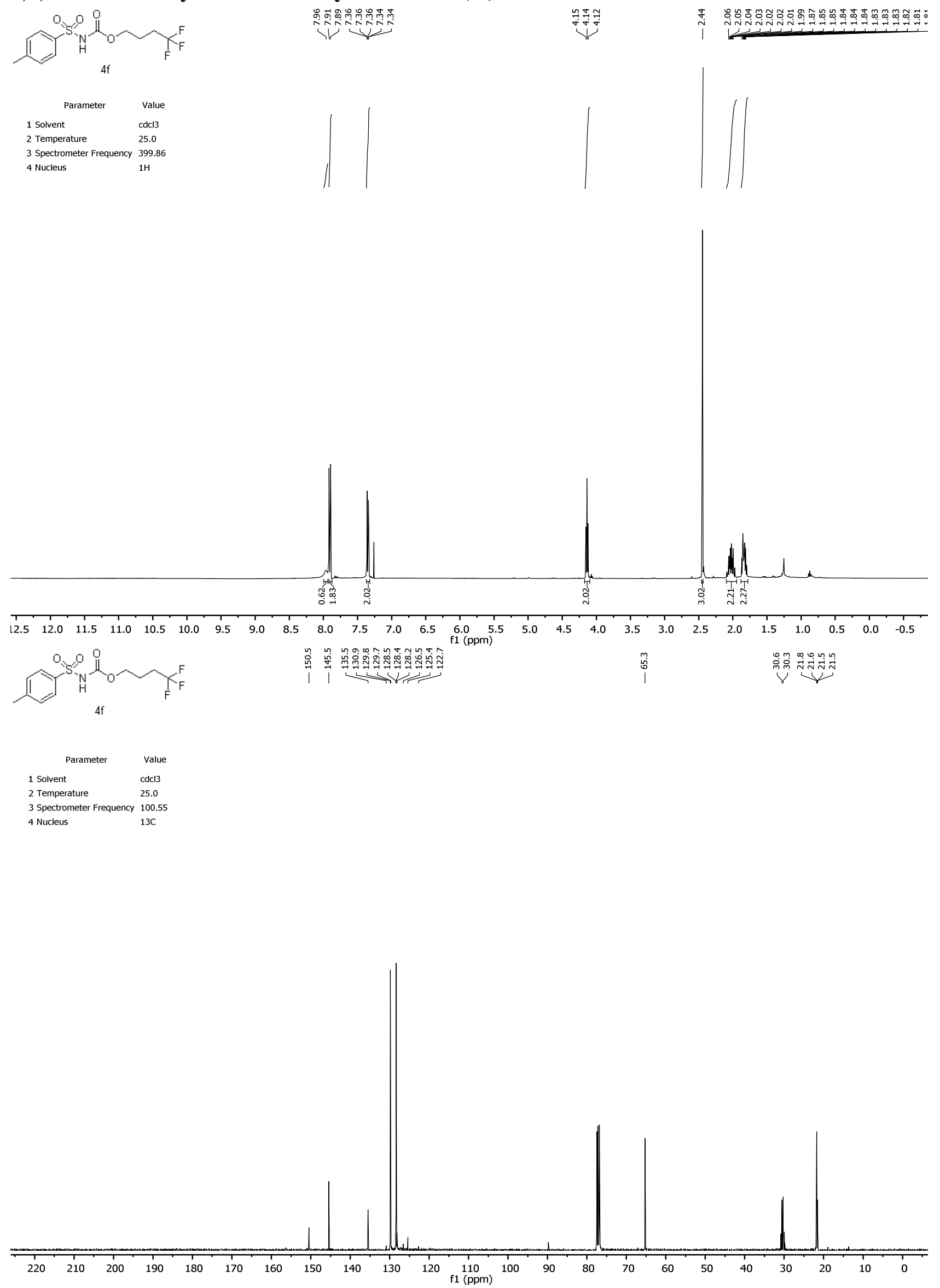
Prop-2-yn-1-yl toluenesulfonylcarbamate (4g)
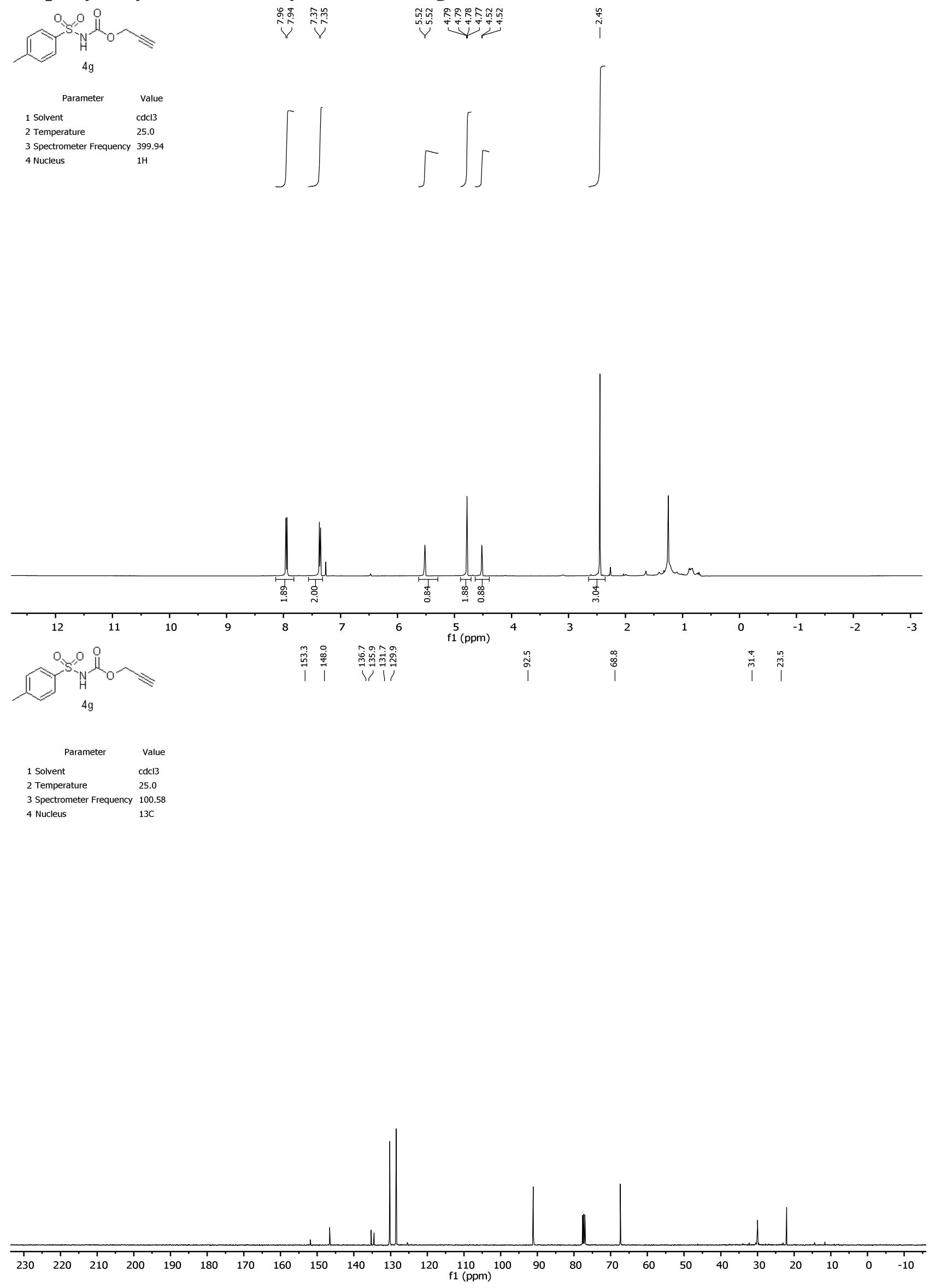
Thiophen-2-ylmethyl toluenesulfonylcarbamate (4h)

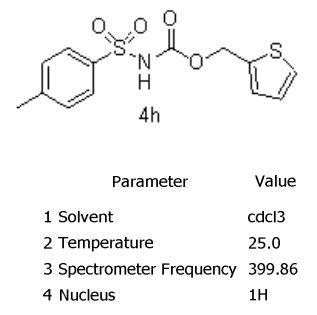

$\underbrace{20}$
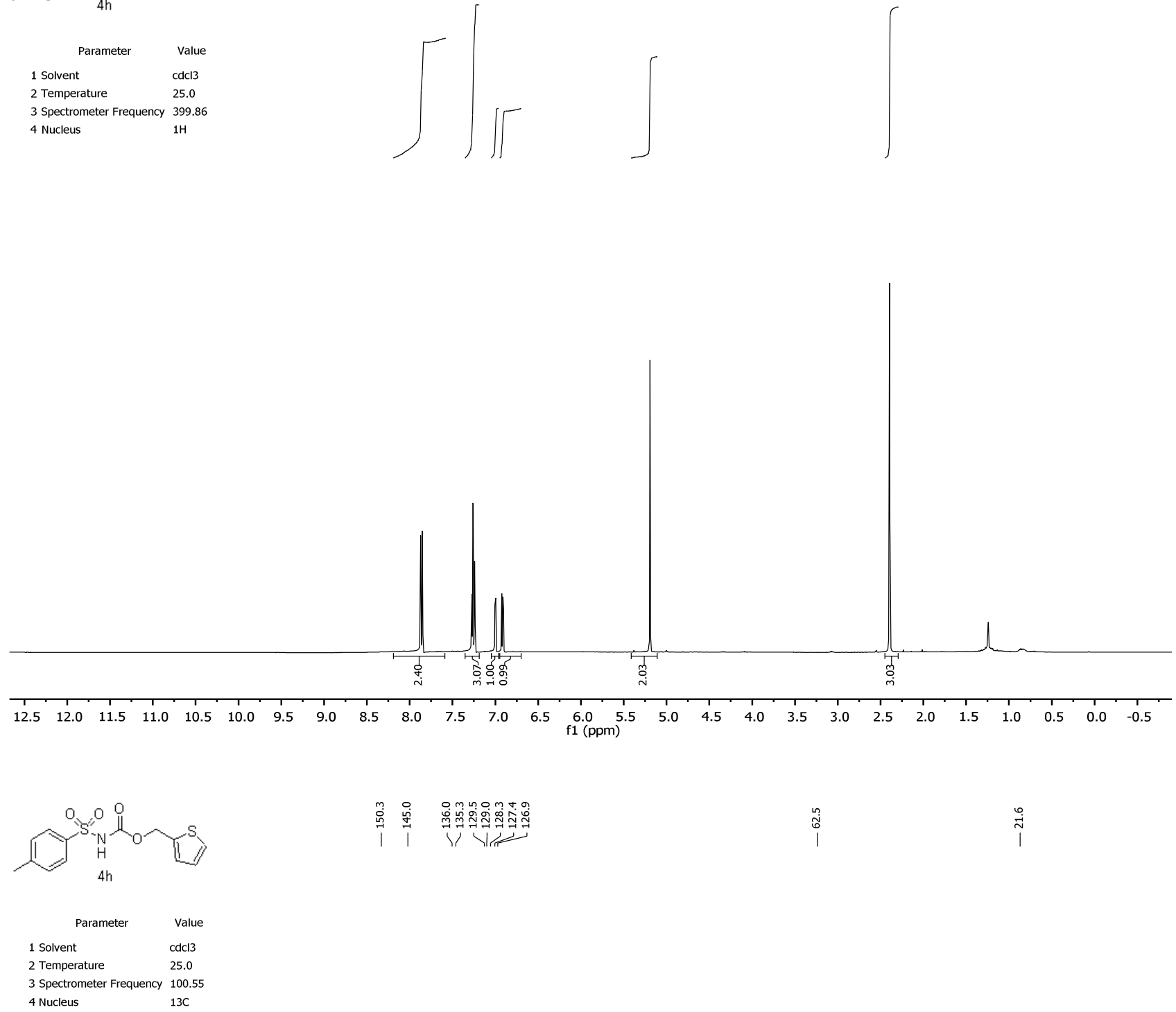

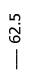

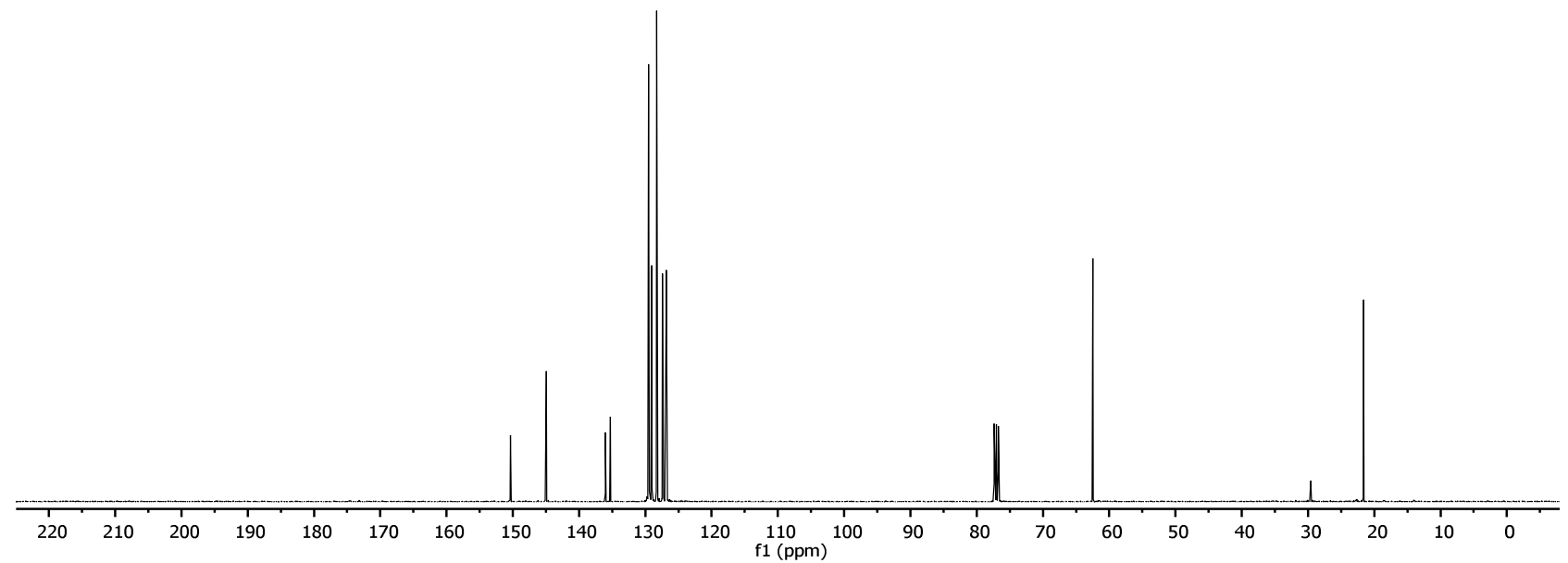


2,3-Dihydro-1H-inden-1-yl toluenesulfonylcarbamate (4i)
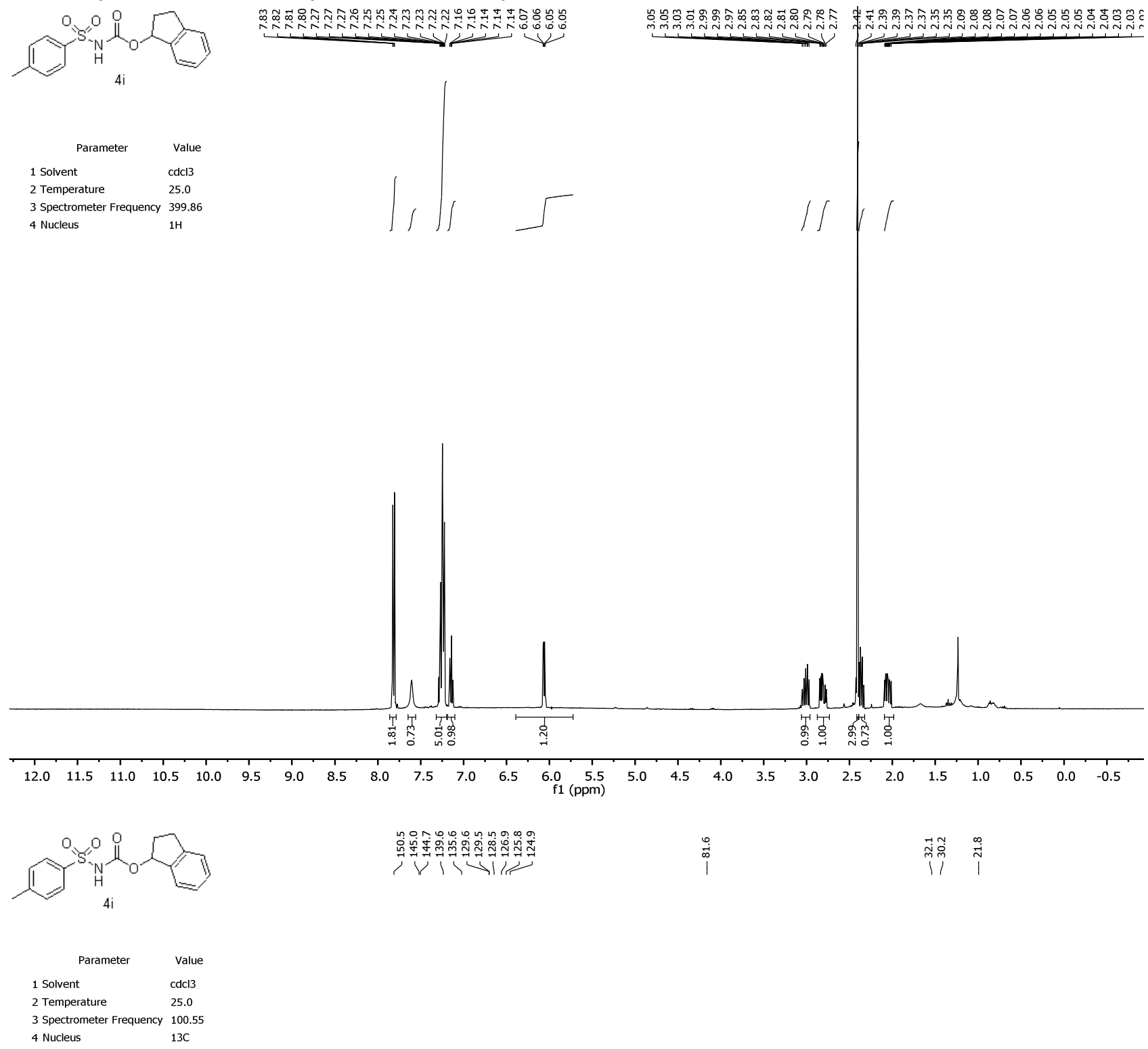

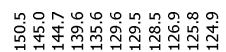

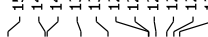

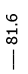

iํㄹํํำ

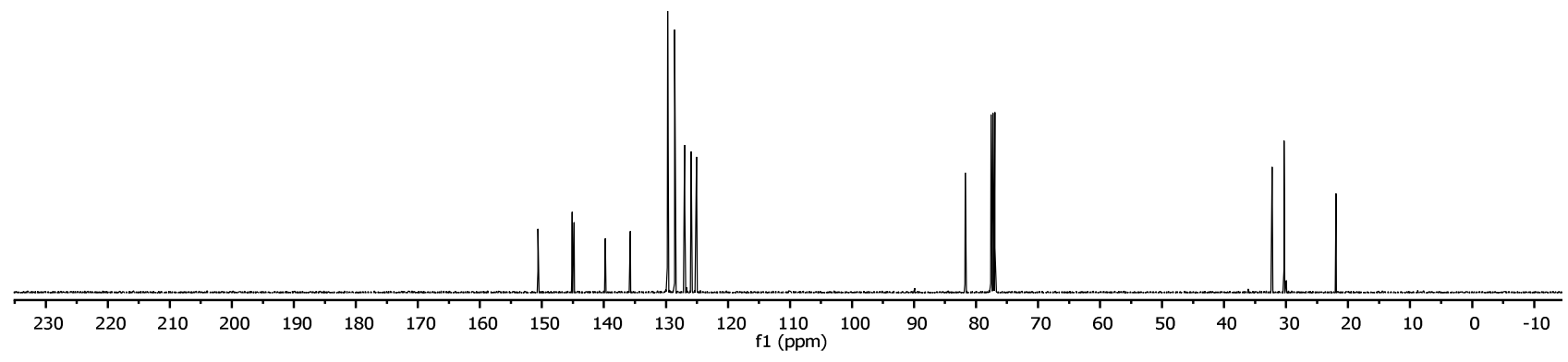




\section{Benzyl toluenesulfonylcarbamate $(\mathbf{4 j})$}

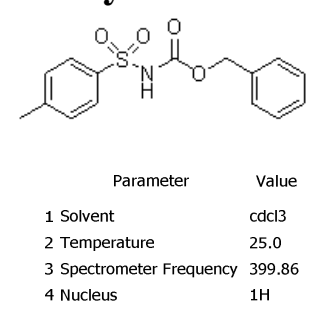

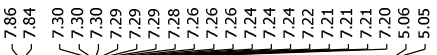

4 Nucleus $\quad 1 \mathrm{H}$
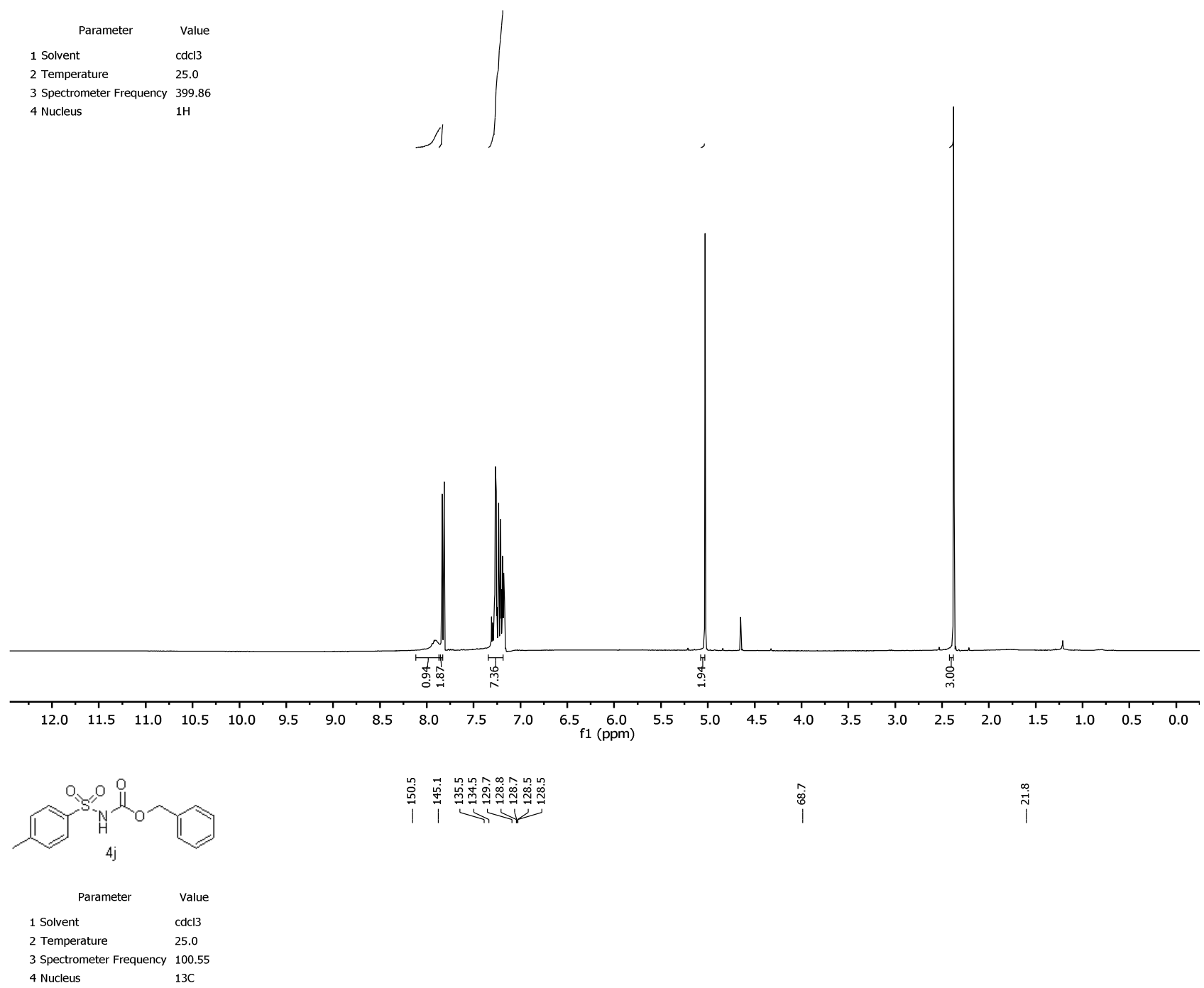

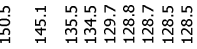

3 Spectrometer Frequency 100.55

4 Nucleus

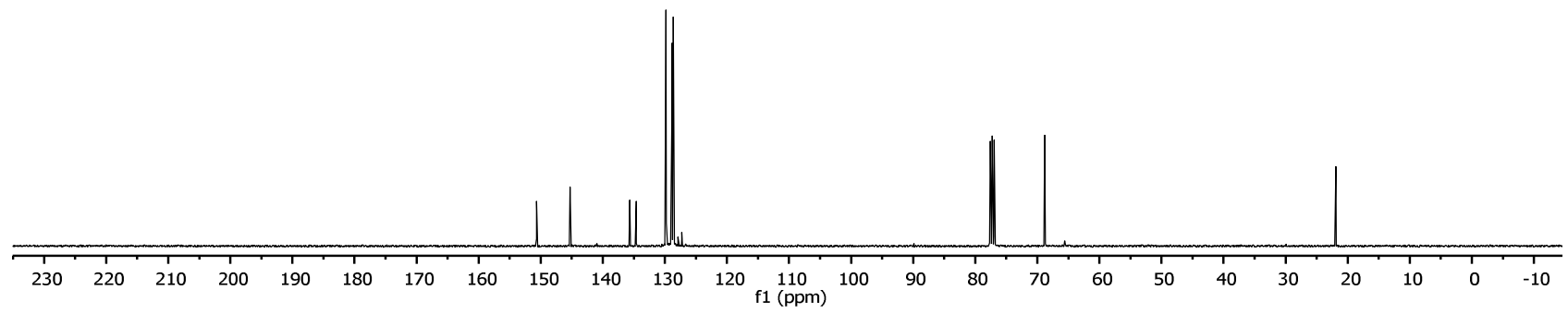


Pyridin-3-ylmethyl toluenesulfonylcarbamate (4k)
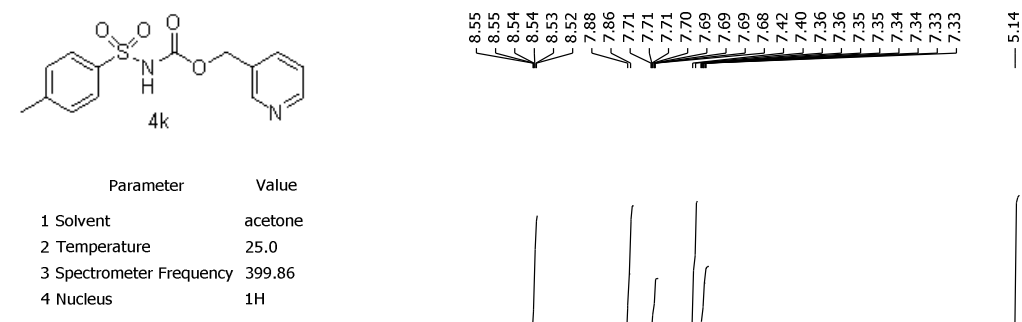

3 Spectrometer Frequency 399.86

4 Nucleus
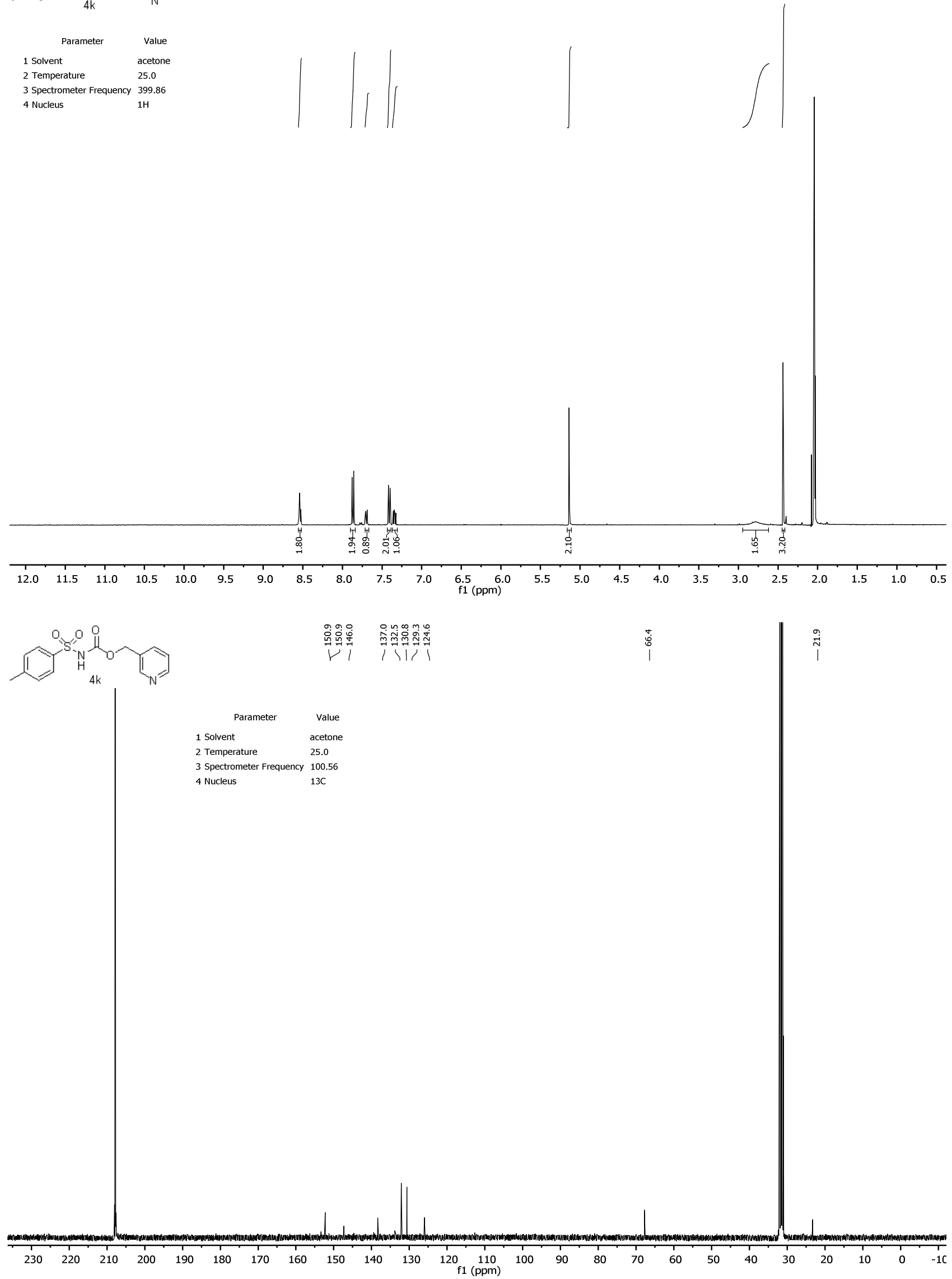
Triethylammonium ((cinnamyloxy)carbonyl)(tosyl)amide (4l)
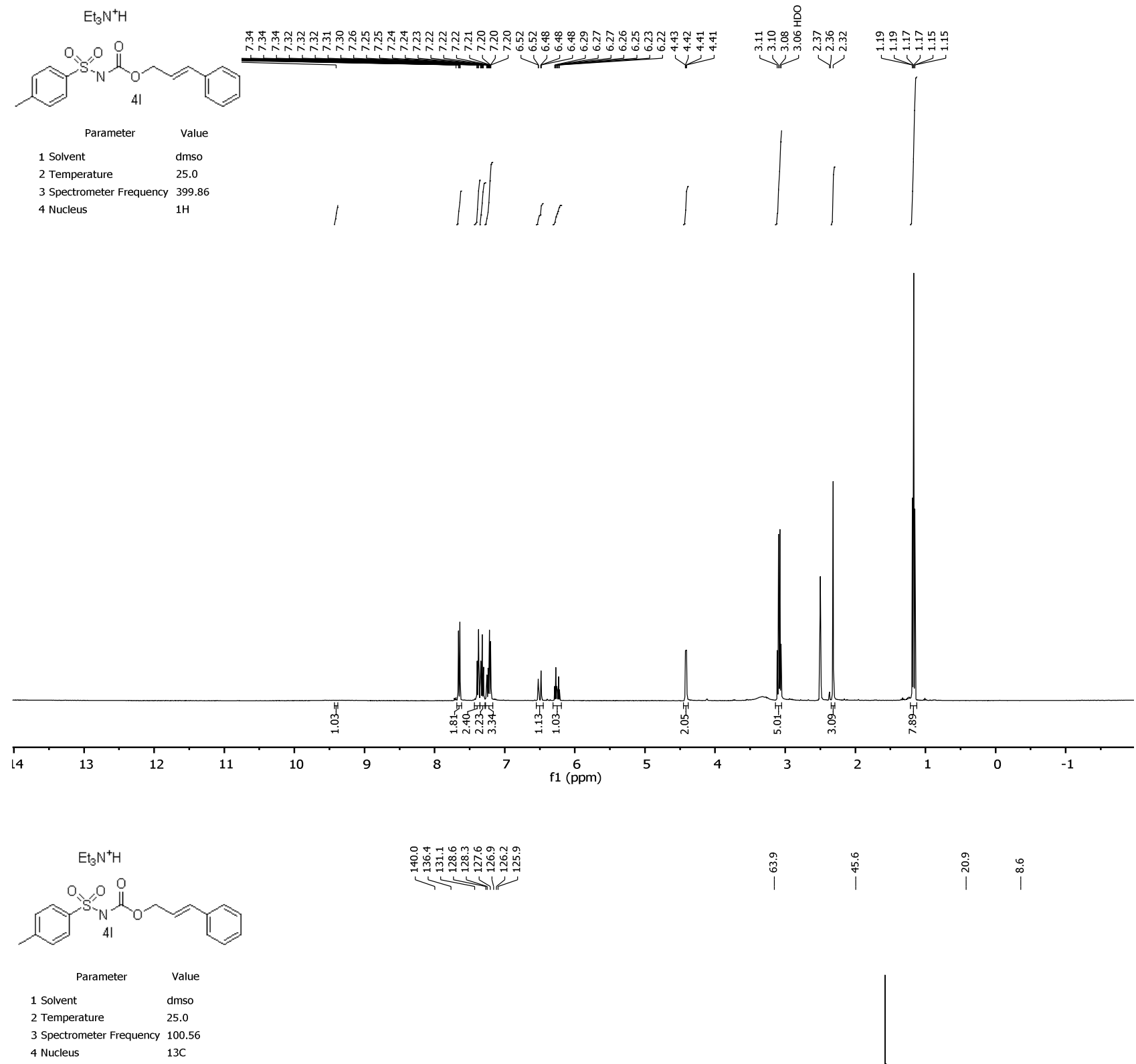

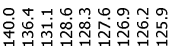

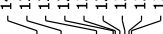

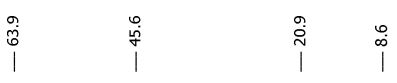

4 Nucleus $13 \mathrm{C}$ 
Triethylammonium (butoxycarbonyl)((4-methoxyphenyl)sulfonyl)amide (4n)
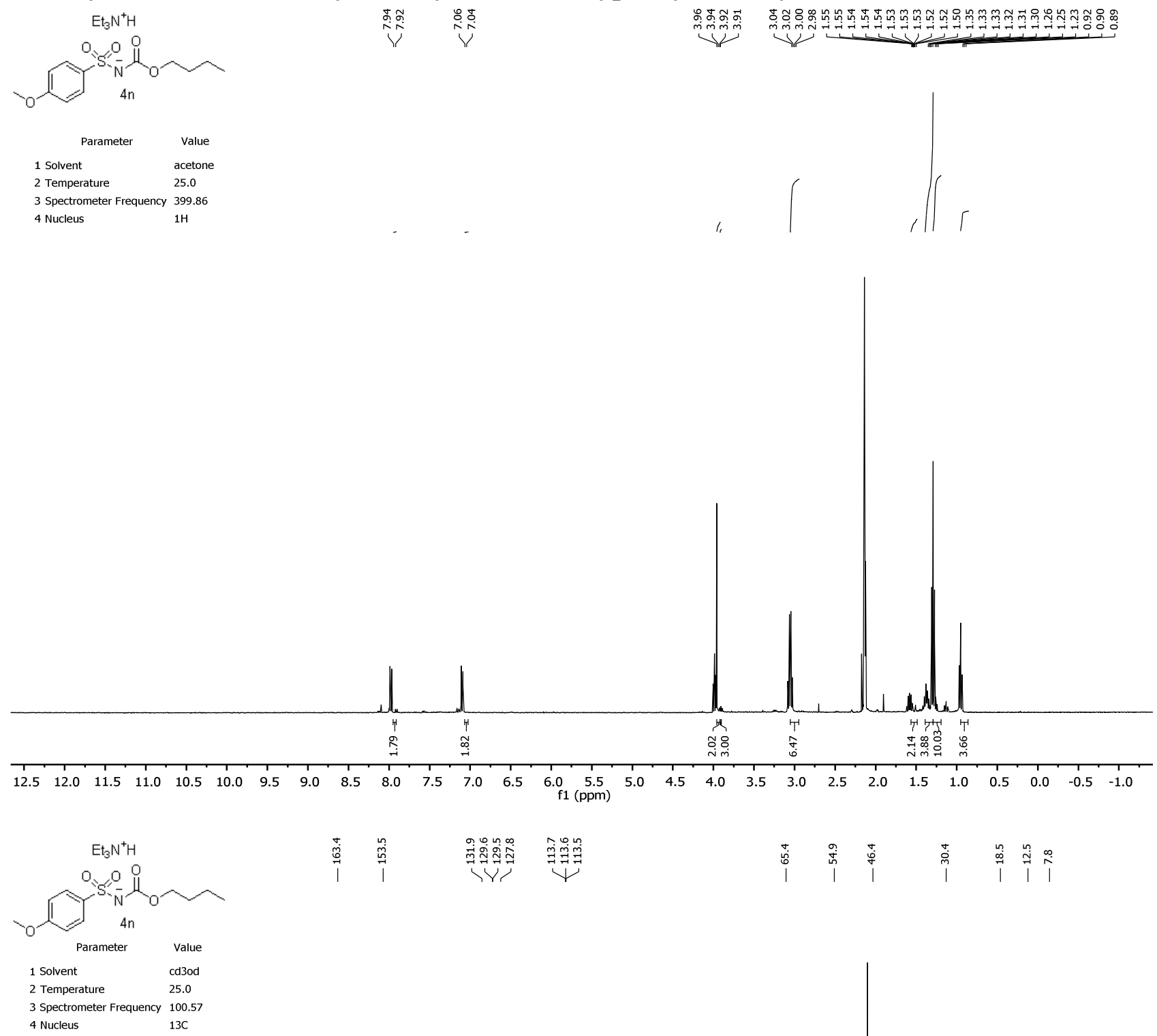

|

i.j.

Nucleus

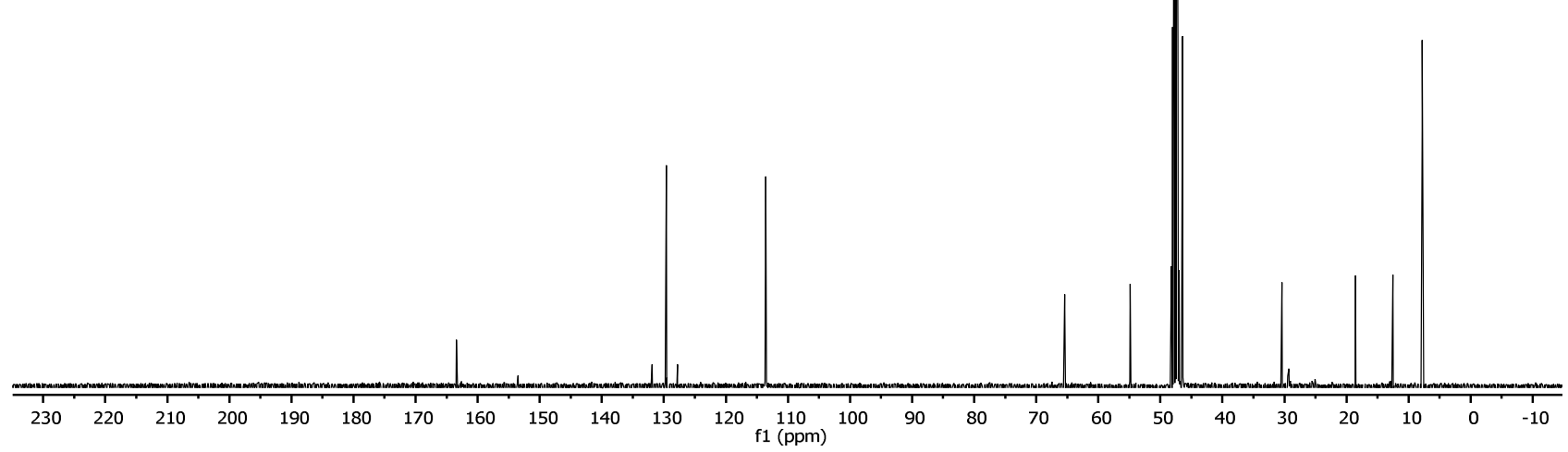


Butyl ((4-acetylphenyl)sulfonyl)carbamate (4o)

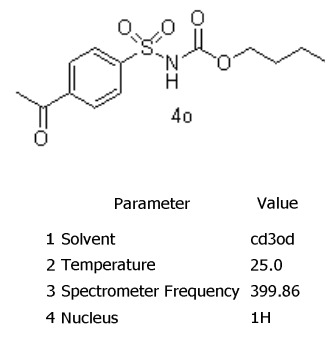

4 Nucleus $\quad 1 \mathrm{H}$
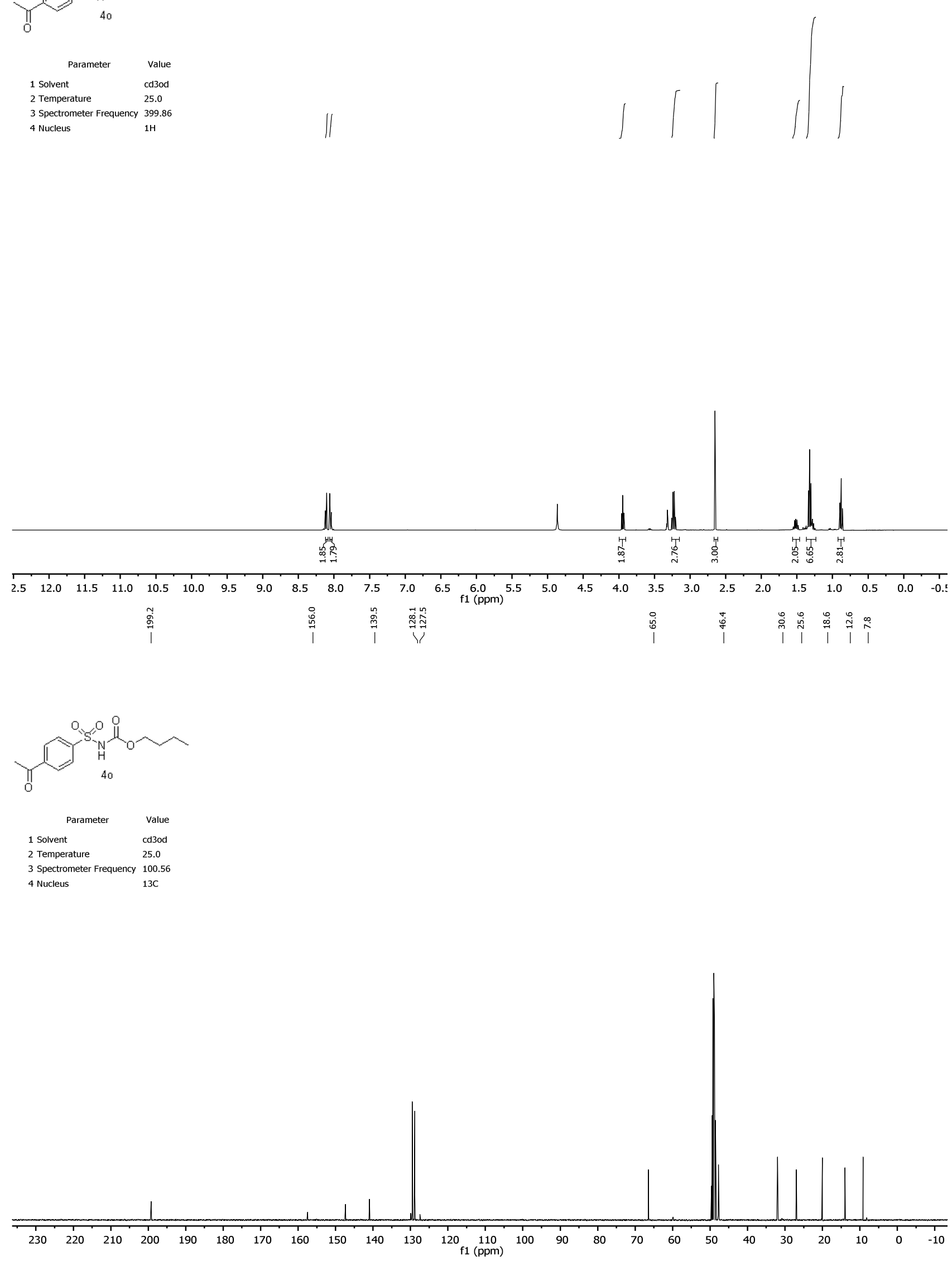
Butyl ((4-bromophenyl)sulfonyl)carbamate (4p)
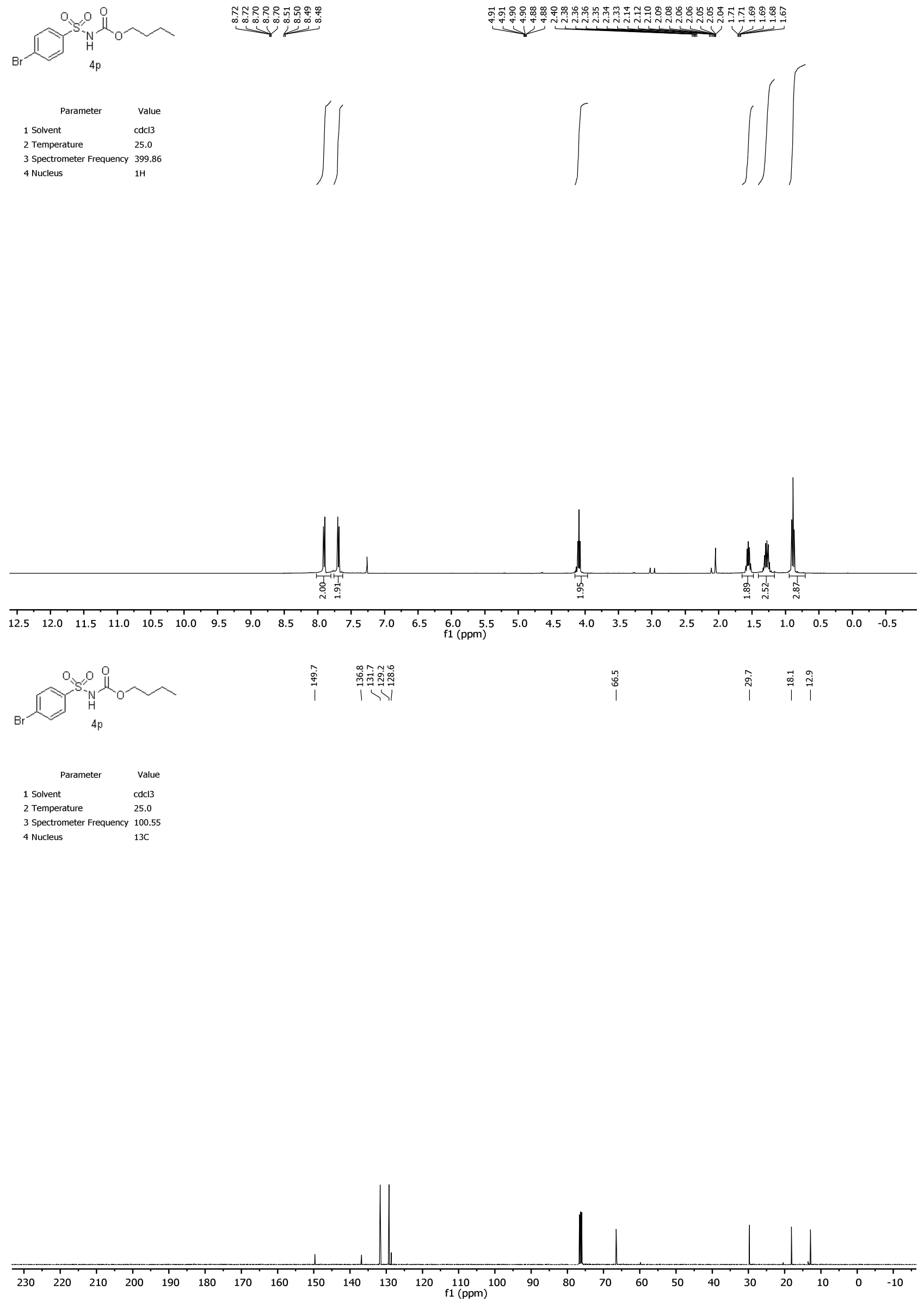
Butyl ((2-bromophenyl)sulfonyl)carbamate (4q)
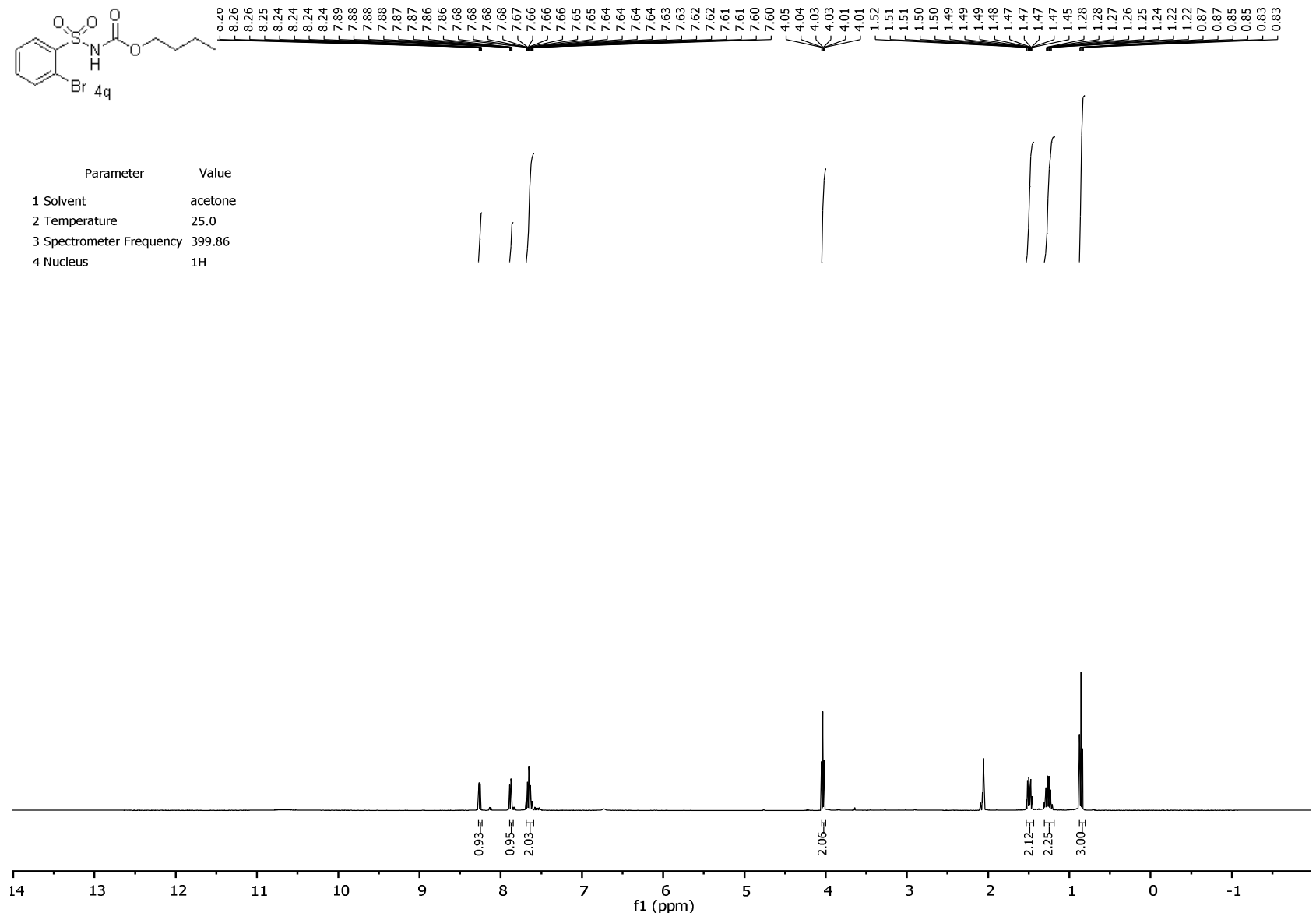

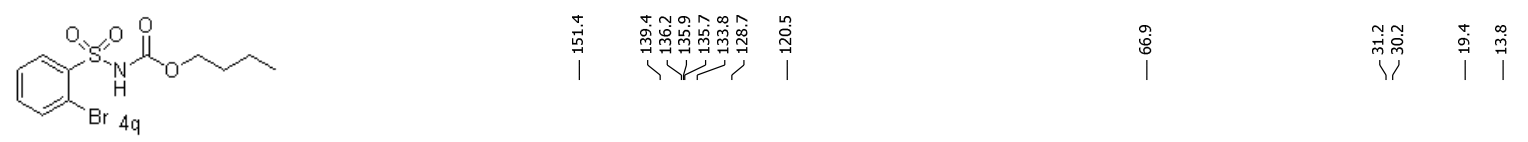

$$
\begin{array}{ll}
\multicolumn{1}{c}{\text { Parameter }} & \multicolumn{1}{c}{\text { Value }} \\
\text { 1 Solvent } & \text { acetone } \\
\text { 2 Temperature } & 25.0 \\
\text { 3 Spectrometer Frequency } & 100.56 \\
\text { 4 Nucleus } & 13 \mathrm{C}
\end{array}
$$

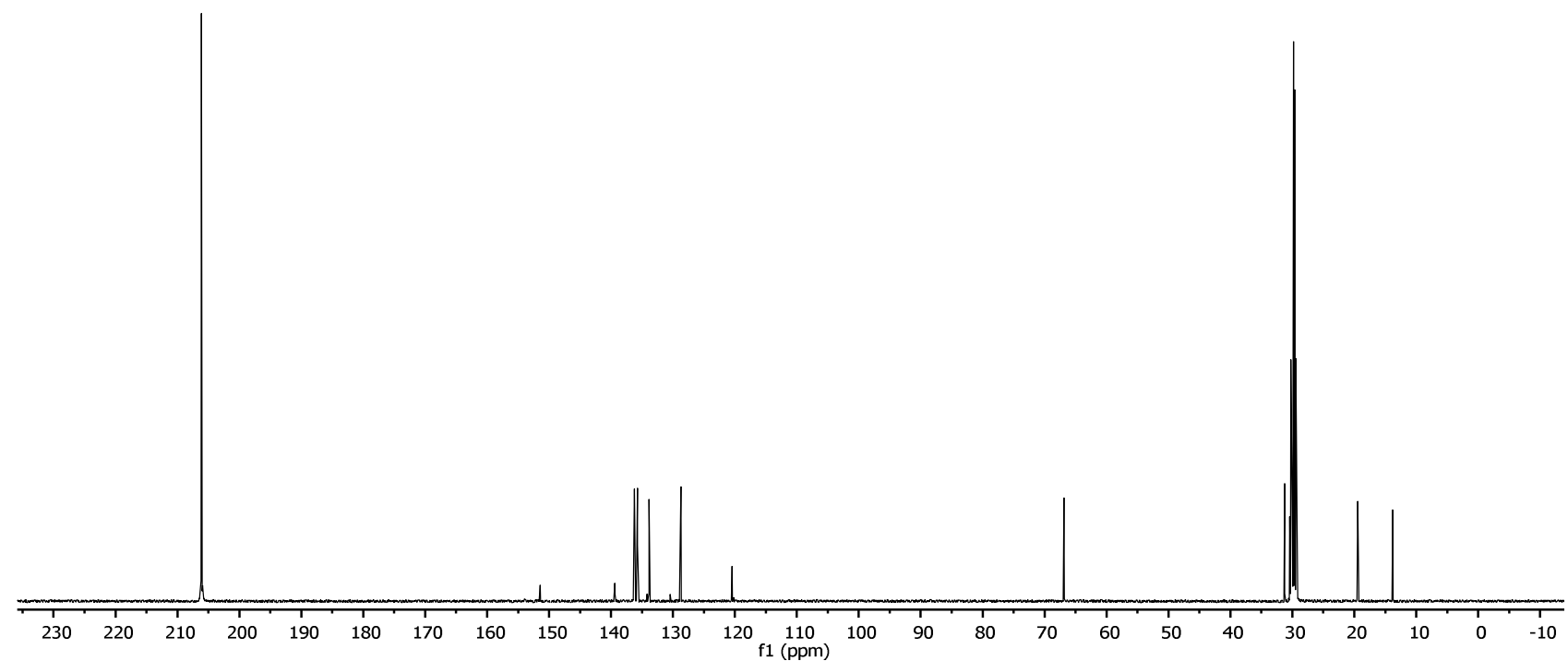


Triethylammonium (butoxycarbonyl)(thiophen-2-ylsulfonyl)amide (4r)

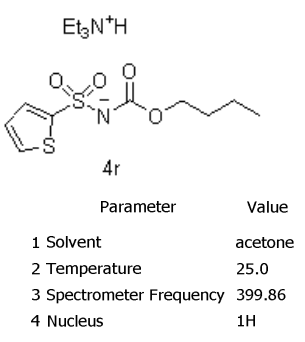

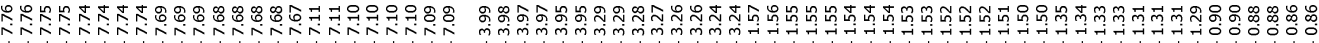
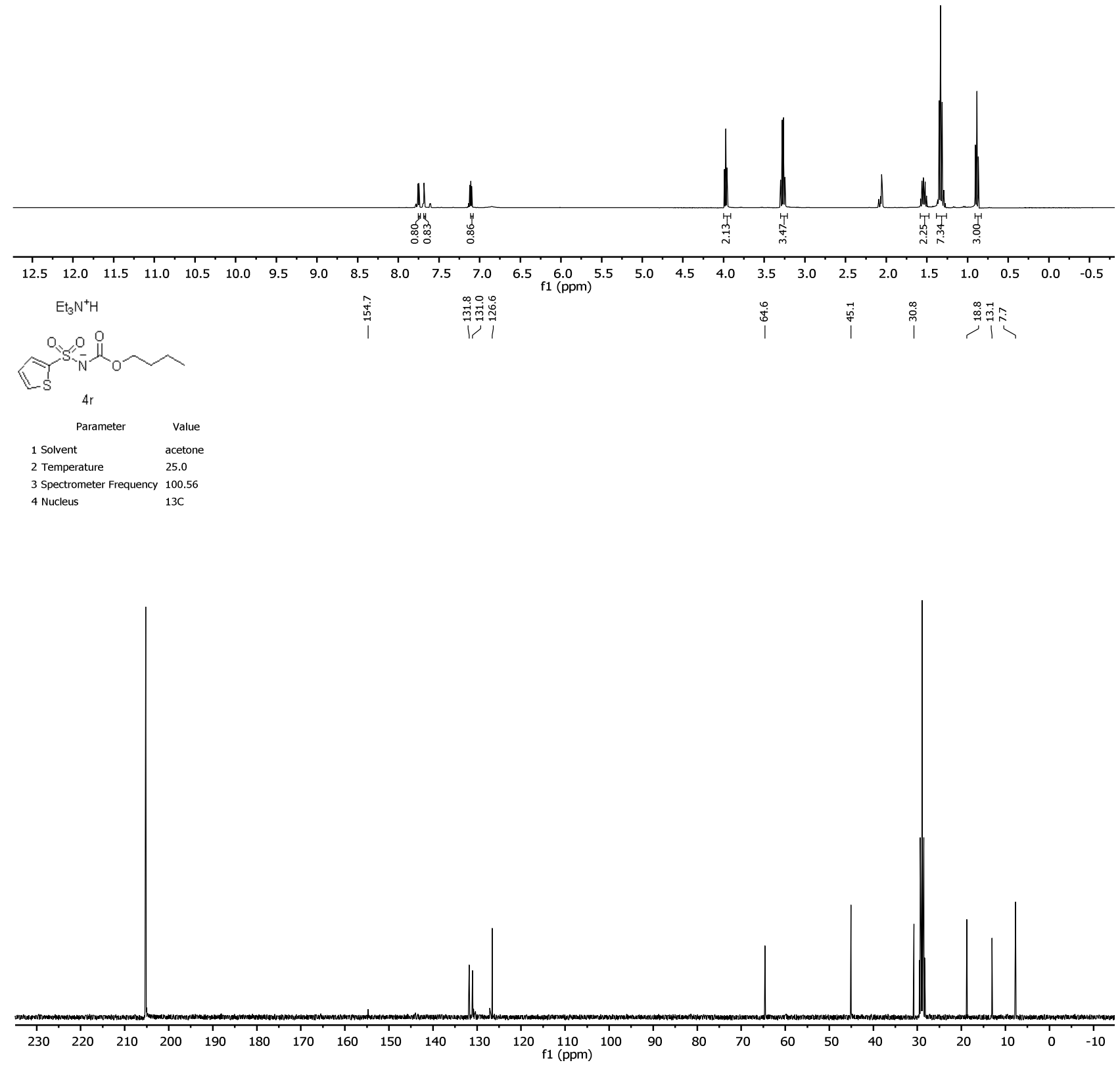
Triethylammonium (butoxycarbonyl)((5-chlorothiophen-2-yl)sulfonyl)amide (4s)
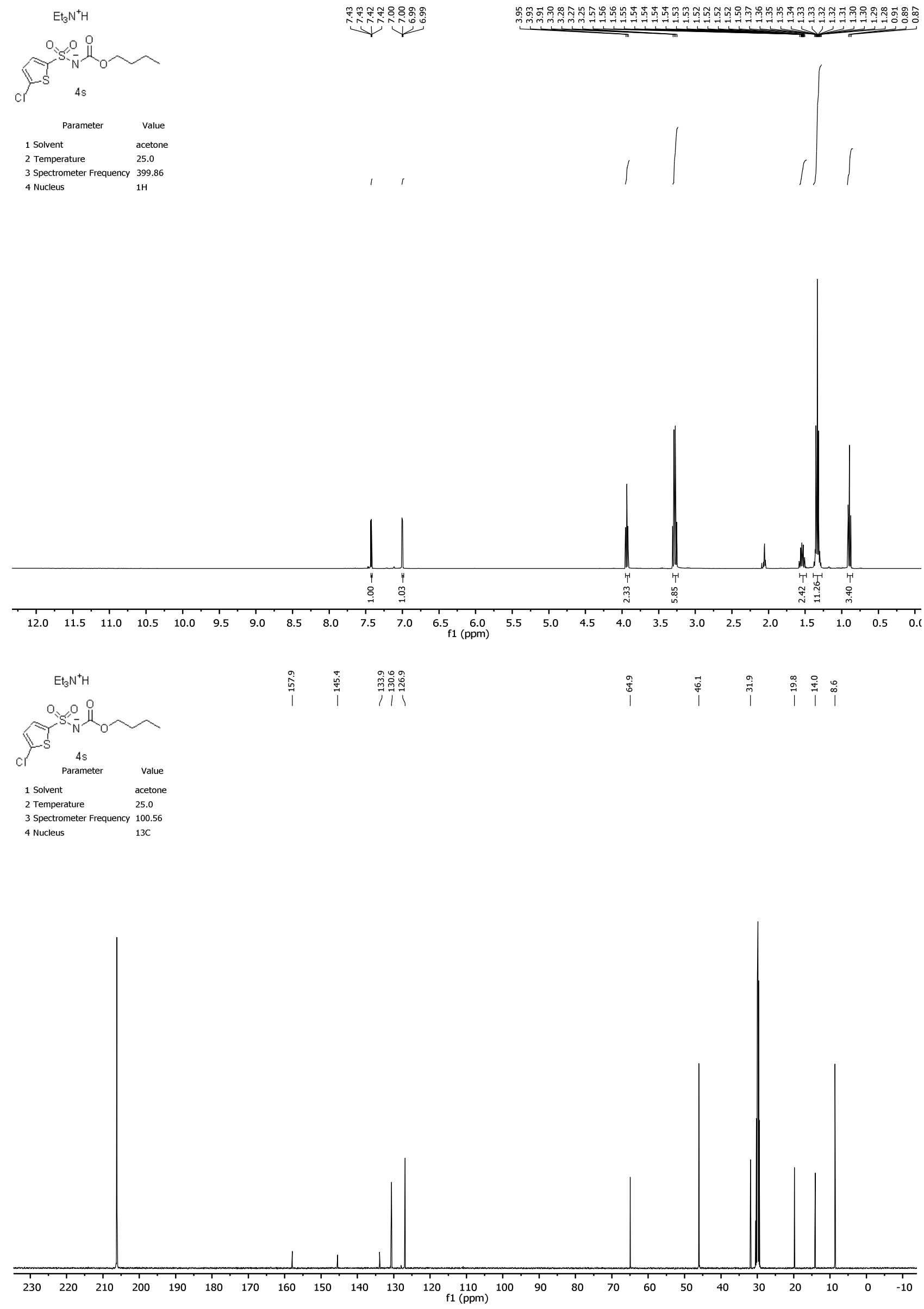

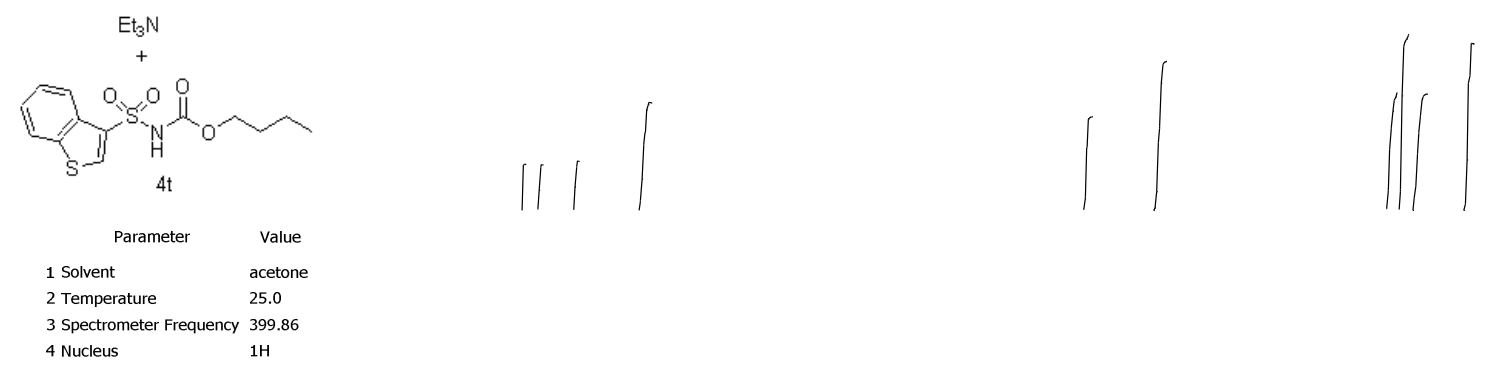

$\begin{array}{ll}3 \text { Spectrometer Frequency } 399.86 \\ 4 \text { Nucleus } & 1 \mathrm{H}\end{array}$
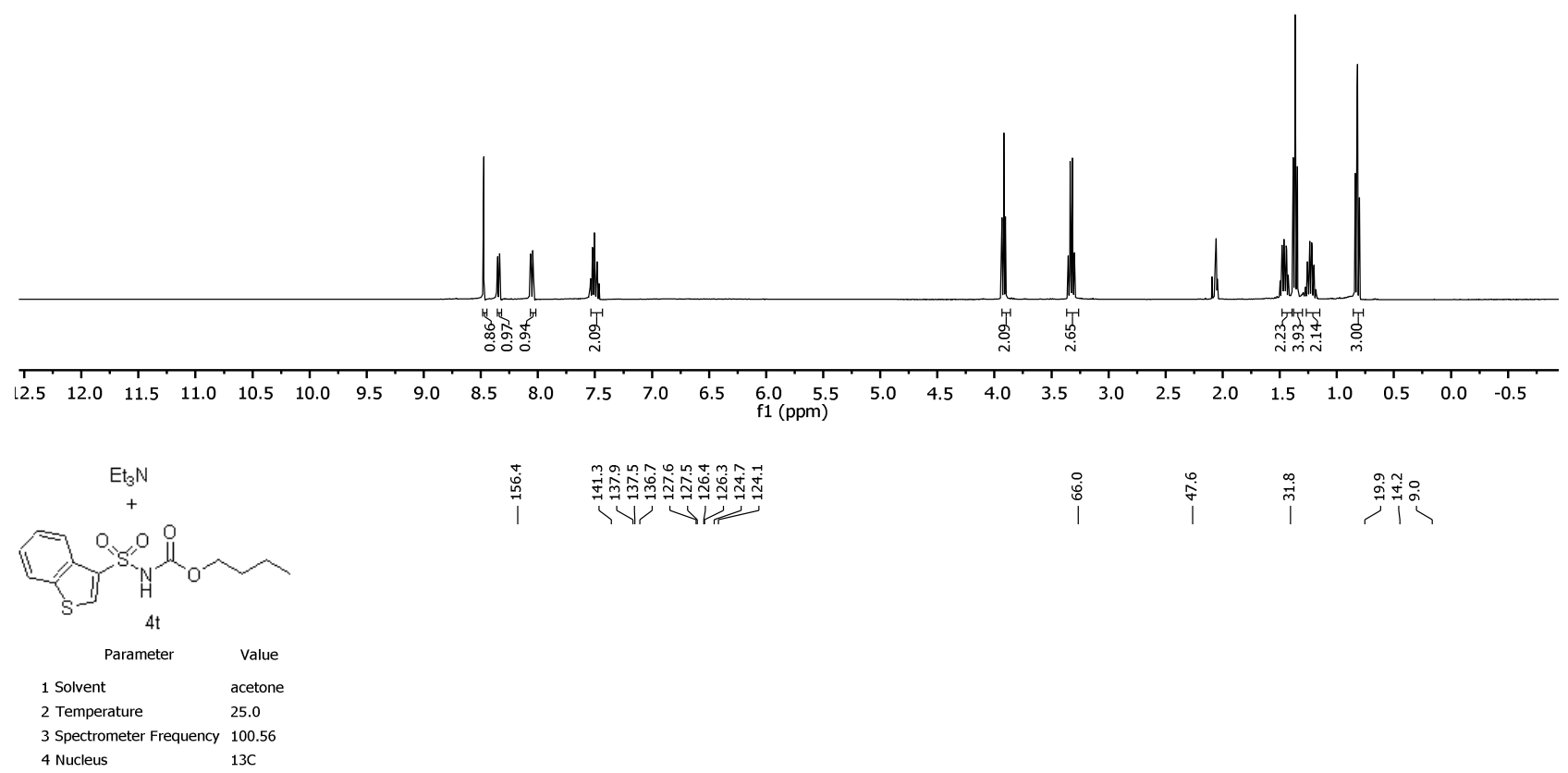

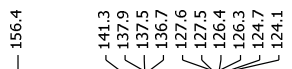

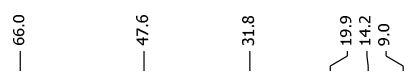

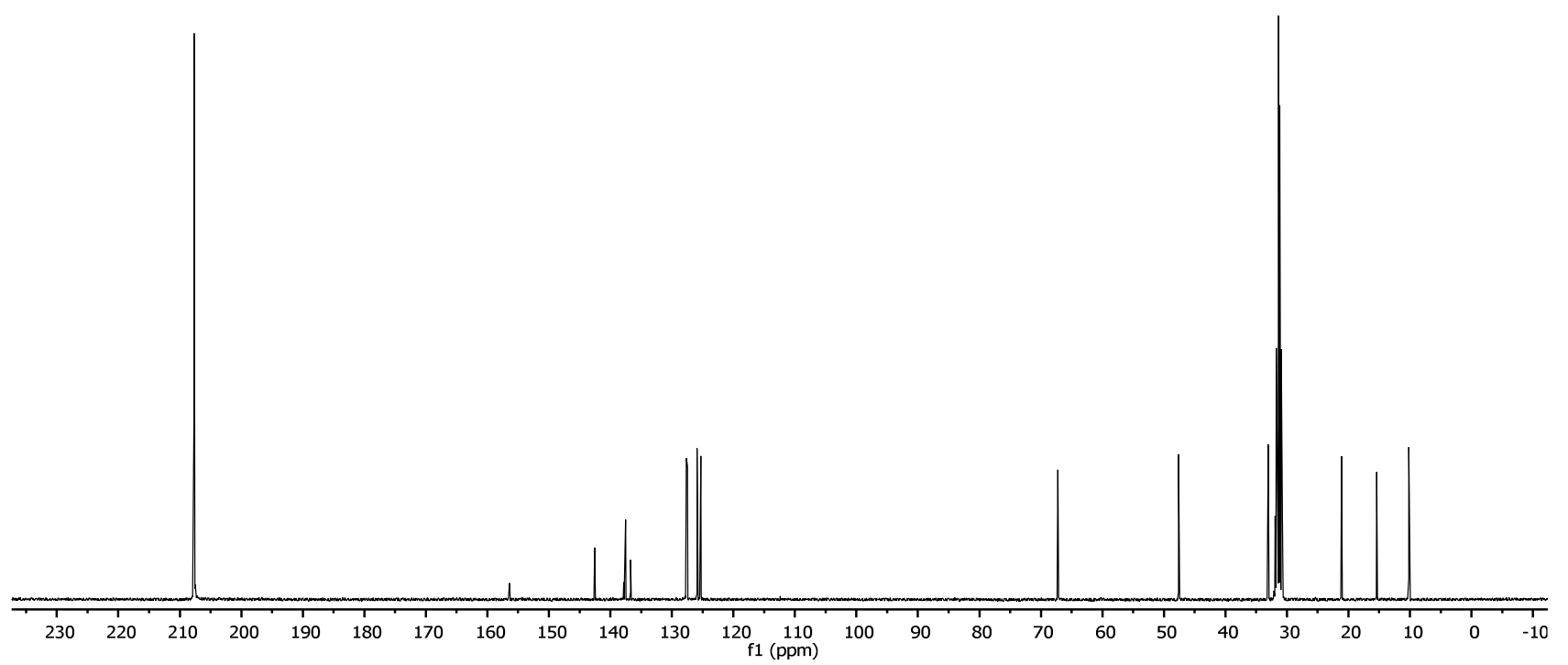


Butyl (benzylsulfonyl)carbamate (4u)
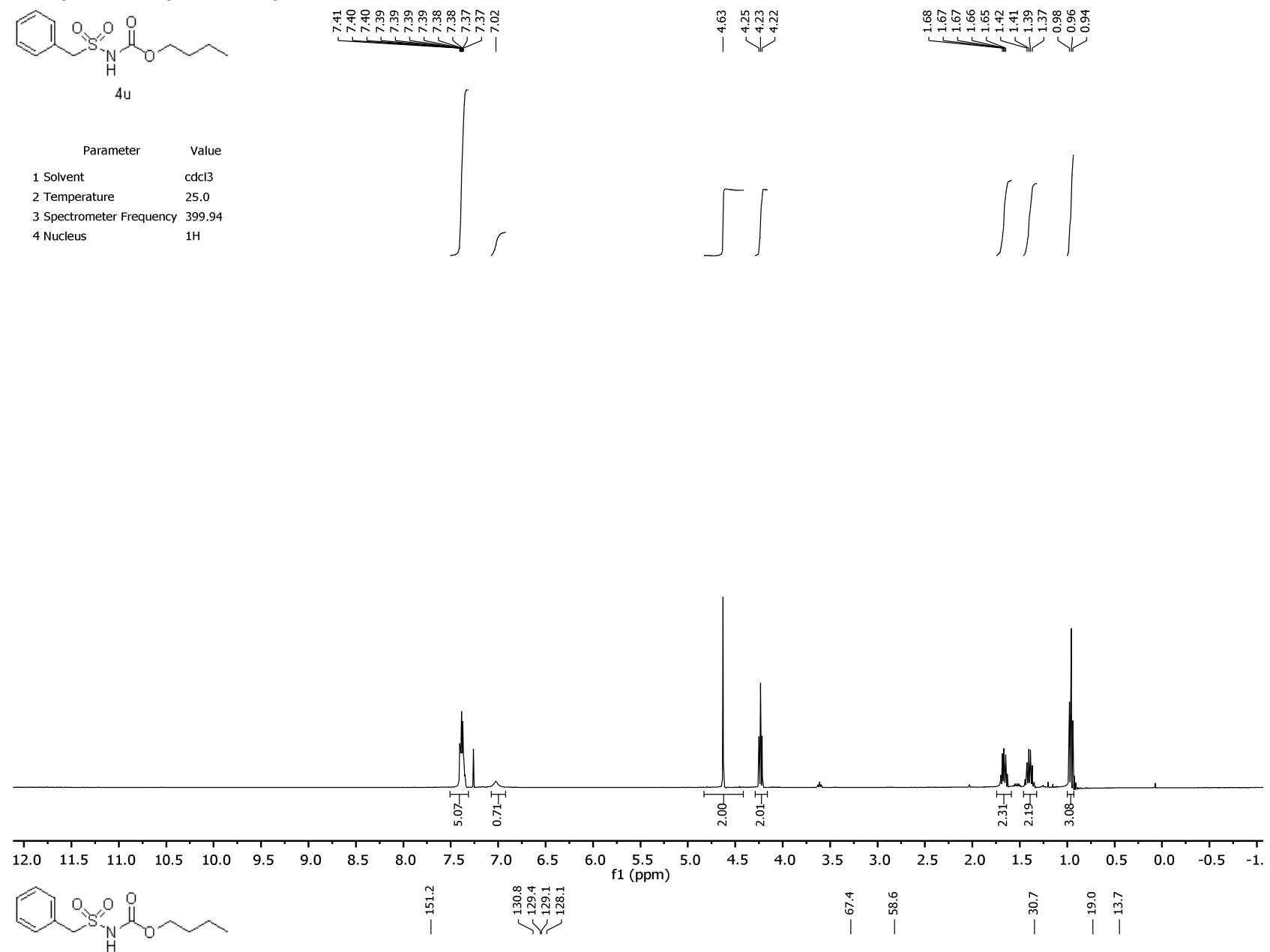

4
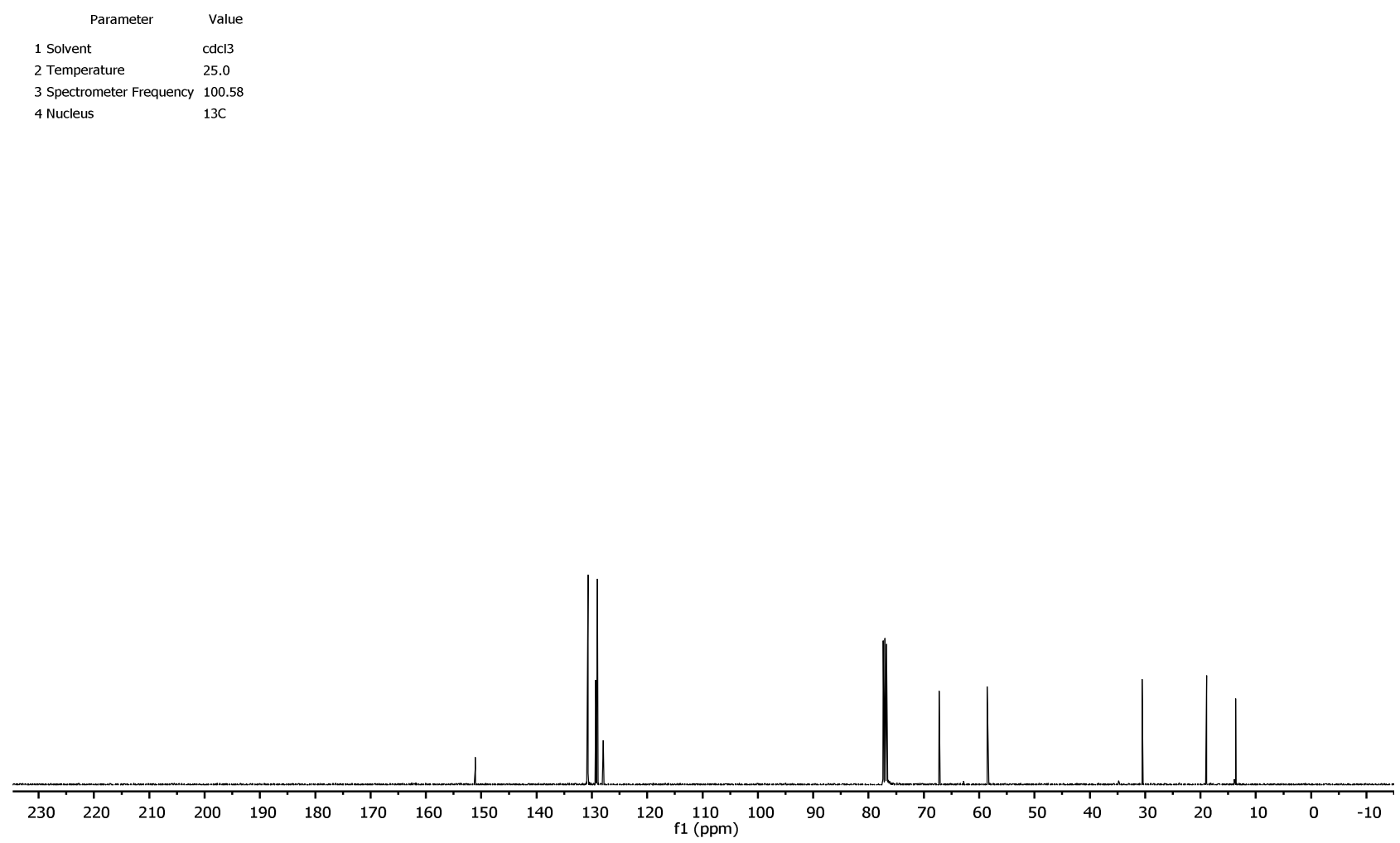
Butyl tosylcarbamate- ${ }^{15} \mathrm{~N}$
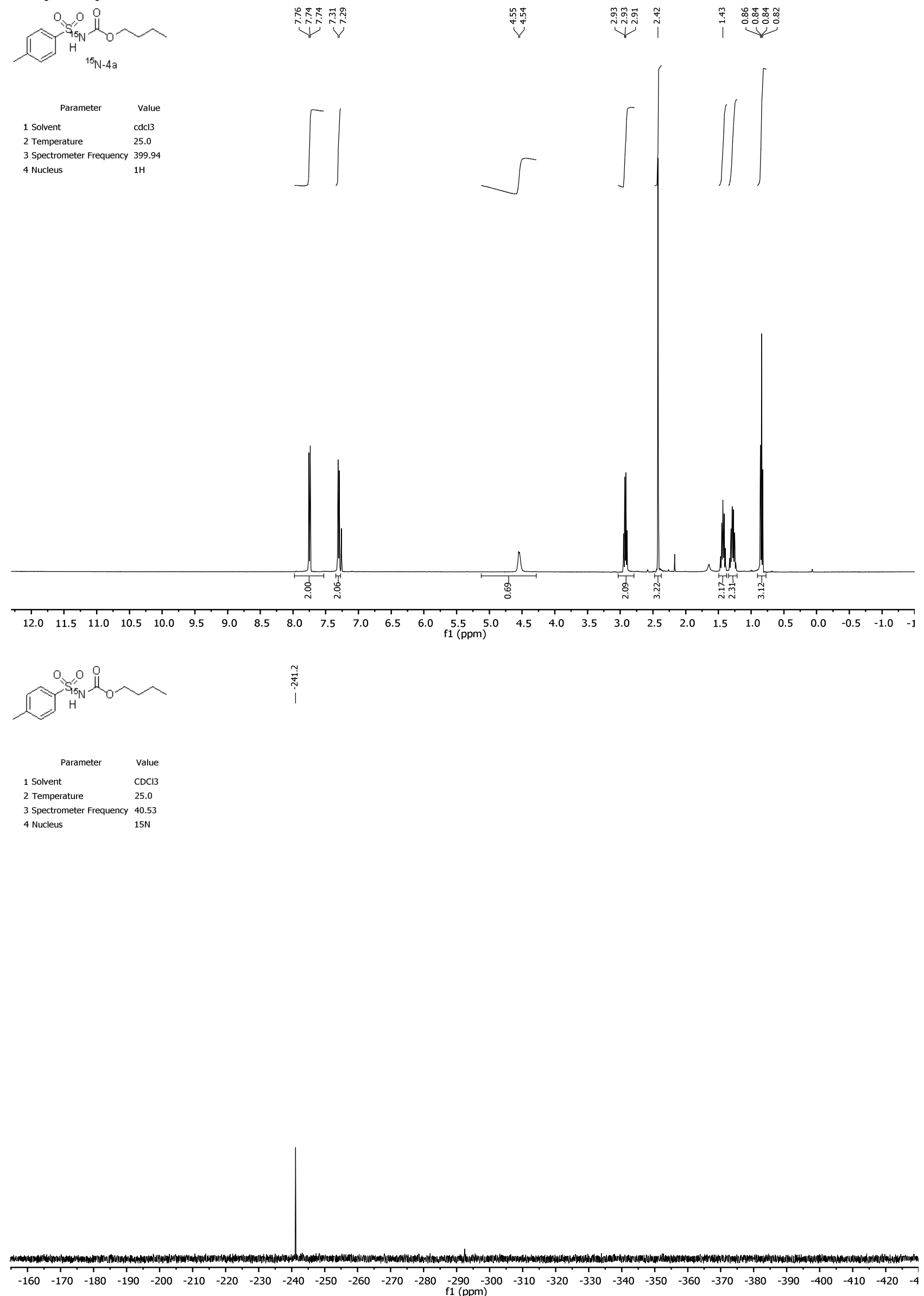


\section{Azide displacement experiment (compound 2a)}

Compound $2 a$, from non-labelled tosyl

azide

Compound $2 \mathrm{a}$, from nitrogen-15

labelled tosyl azide

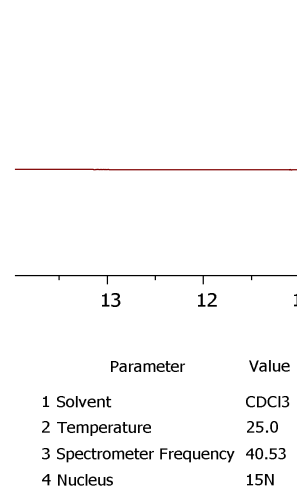

4 Nucleus

$15 \mathrm{~N}$

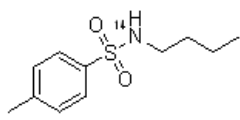

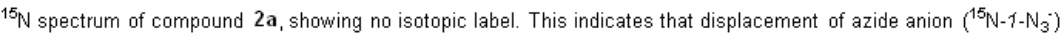

has occurred 